NASA CONTRACTOR REPOR T

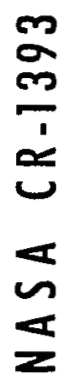

LOAN COPY: RETURN TO

AFWL (WLIL-2)

KIRTLAND AFB, N MEX

EXPERIMENTAL INVESTIGATION

OF MAGNETOAERODYNAMIC FLOW

AROUND BLUNT BODIES

by S. Kranc, M. C. Yuen, and A. B. Cambel

Prepared by

NORTHWESTERN UNIVERSITY

Evanston, IIl.

for

NATIONAL AERONAUTICS AND SPACE ADMINISTRATION - WASHINGTON, D. C. - AUGUST 1969 


\title{
EXPERIMENTAL INVESTIGATION OF
}

\section{MAGNETOAERODYNAMIC FLOW AROUND BLUNT BODIES}

\author{
By S. Kranc, M. C. Yuen, and A. B. Cambel
}

Distribution of this report is provided in the interest of information exchange. Responsibility for the contents resides in the author or organization that prepared it.

Prepared under Grant No. NsG-547 by

NORTHWESTERN UNIVERSITY

Evanston, $\mathrm{nl}$.

for

NATIONAL AERONAUTICS AND SPACE ADMINISTRATION

For sale by the Clearinghouse for Federal Scientific and Technical Information Springfield, Virginia 22151 - CFSTI price $\$ 3.00$ 


\section{ABSTRACT}

The drag on a body with a self-contained electromagnet are measured with the body is placed in a low density argon plasma stream. Tests are conducted in a steady flow of plasma produced in a direct current arc and expanded to supersonic speeds. Measurements are restricted to one flow condition where the influence of viscosity and the Hall effect are both important.

From experimental measurements of the total drag on the body, the Lorentz force on the magnet and the pressure force on the body, the viscous forces on the body may be deduced. Empirical relations for the data are then generated. It is found that the total drag and Lorentz force increase with increasing magnetic fleld but that the pressure and viscous forces decrease. A surprising result is that pressure and density increase at the stagnation point with magnetic field contrary to previous predictions. This indicates that when Hall effect is important, the assumption of constant density in the shock layer is no longer justified. The experimental results are compared to existing theoretical treatments of the problem.

The flow field around the body is investigated spectroscopically, and the predicted increase in shock stand-off distance is observed photographically. An unexpected visible color change which occurs when the magnetic field is applied is also investigated. Spectroscopic measurements show that the electrons in the plasma are out of thermal equilibrium in the plasma and the flow is essentially non reacting. The influence of these facts on other experimental measurements is considered. 
$-25$ 
TABLE OF CONTENTS

PAGE

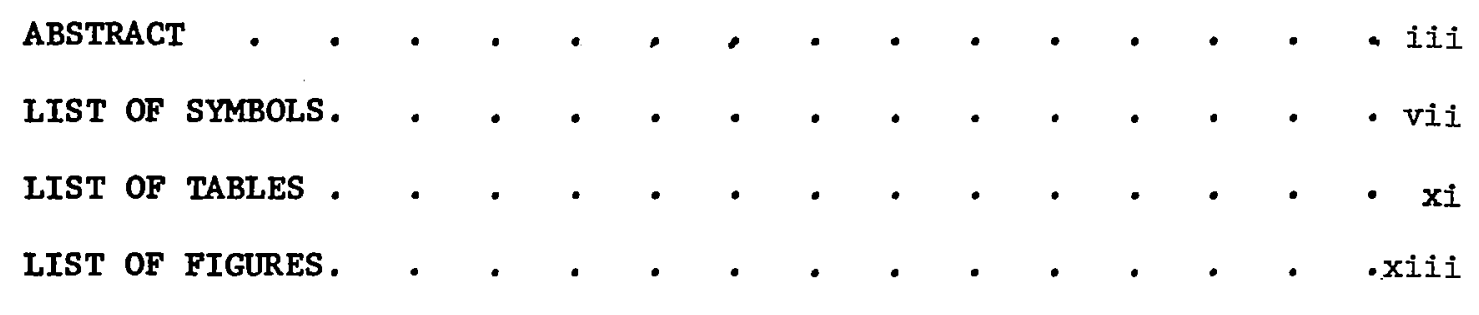

CHAPTER

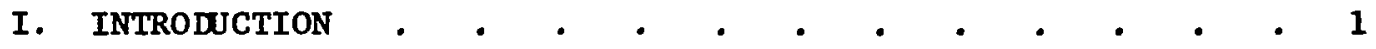

1.1 Literature Survey 4

1.2 Description of Magnetoaerodynamic Flow 10

II. CONSIDERATIONS IN THE MEASUREMENT OF DRAG AND SHOCK

STANDOFF. . . . . . . . . . . . . 16

2.1 The Components of Magnetoaerodynamic Drag 16

2.2 Previous Experimenta1 Work 20

2.3 Preliminary Experiments $\quad 22$

III. THE EXPERIMENTAL FACILITY AND EVALUATION OF FLOW CONDITIONS. 25

3.1 The Experimental Facility 25

3.2 Choice of Flow Condition 27

3.3 Diagnostic Measurements of Flow Conditions 29

3.4 Calculation of Flow Qauntities 31

3.5 Calculation of Dimensionless Parameters 36

3.6 Consistency of Data and Error 37

IV. EXPERTMENTAL OBSERVATIONS OF MAGNETOAERODYNAMIC DRAG • 41

4.1 Design, Construction and Calibration of Experimental
Apparatus

4.2 Experimental Measurement of Drag and Drag Components 46

V. INVESTIGATION OF THE SHOCK LAYER . . . . . . . . . 50

5.1 Shocks in a Partially Ionized Gas 50

5.2 The Character of the Shock Layer 53

5.3 Spectroscopic Methods 57

5.4 The Experimental Arrangement 61

5.5 Spectroscopic Measurements and Results 64

5.6 Photographic Measurement of Shock Stand-off Altera-
tion with Magnetic Field

5.7 Observations in the Freestream 69 
VI. DISCUSSION OF RESULTS AND COMPARISON TO THEORY . . . .

6.1 Derivation and Discussion of Empirical Relations for the Data

6.2 Comparison of Magnetoaerodynamic Drag Data to Theory

VII. SUMMARY AND CONCLUSIONS. . . . . . . . . . . 89

REFERENCES ...................... 92

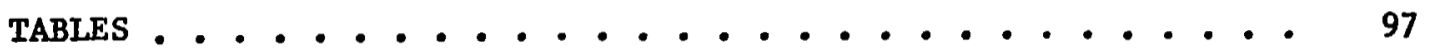

FIGURES. . . . . . . . . . . . . . . . . . 100

APPENDIX A . . . . . . . . . . . . . . . 137

APPENDIX B ..................... 143 


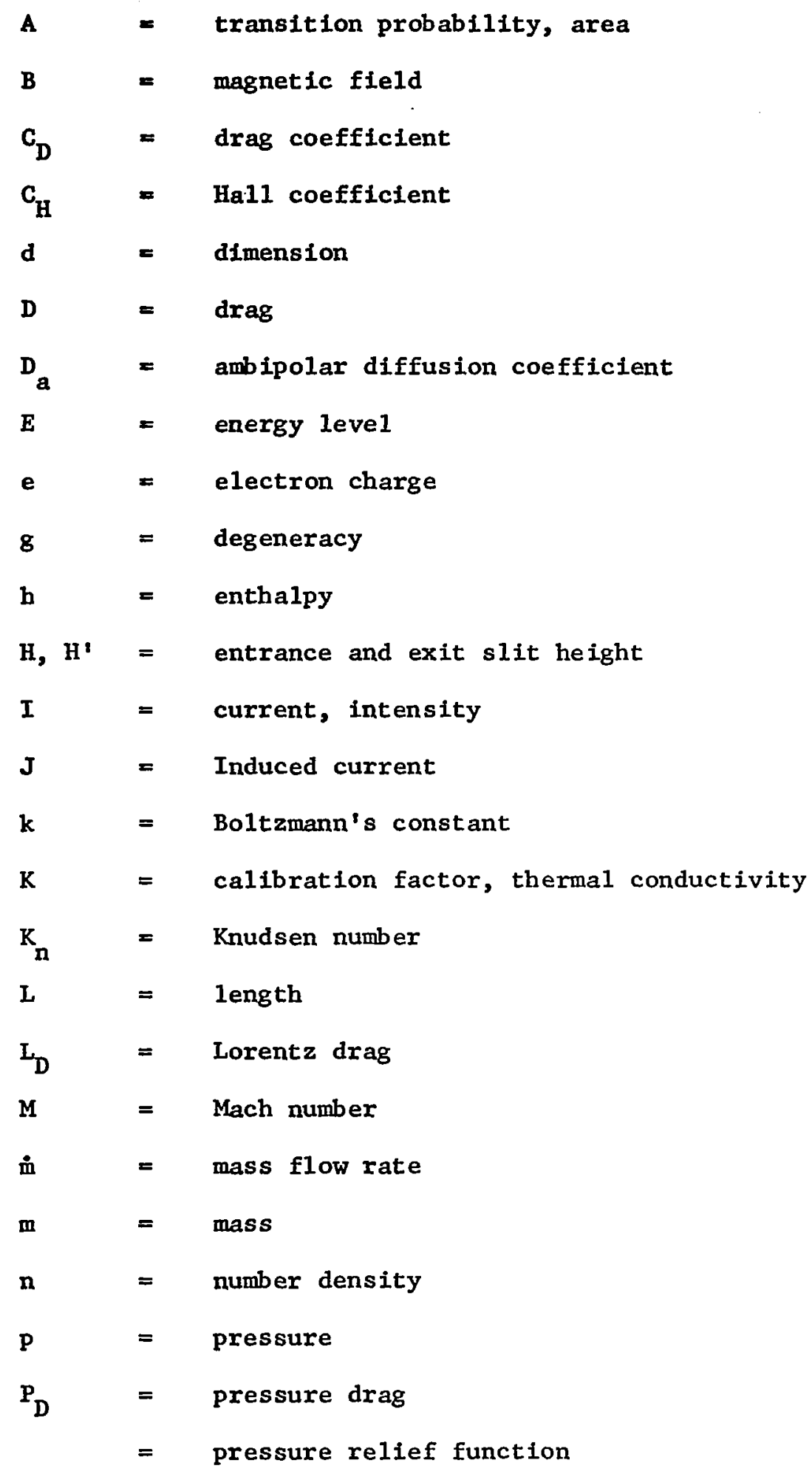




\begin{tabular}{|c|c|c|}
\hline $\mathbf{Q}$ & $=$ & equilibration rate \\
\hline$q$ & $=$ & recombination rate \\
\hline $\mathbf{R}$ & $=$ & radius \\
\hline $\operatorname{Re}$ & $=$ & Reynolds number \\
\hline Rem & $=$ & magnetic Reynolds number \\
\hline $\mathbf{s}$ & $=$ & interaction parameter \\
\hline Sc & $=$ & Schmidt number \\
\hline $\mathbf{t}$ & $=$ & time \\
\hline $\mathbf{T}$ & $=$ & temperature \\
\hline $\mathbf{v}$ & $=$ & macroscopic velocity \\
\hline $\mathbf{v}$ & $=$ & voltage \\
\hline $\mathrm{W}, \mathrm{w}^{\prime}$ & $=$ & entrance and exit slit width \\
\hline $\mathbf{x}$ & $=$ & Ionization potential \\
\hline $\mathbf{z}$ & $=$ & axial position from stagnation point of body \\
\hline$\alpha$ & $=$ & recombination coefficient, degree of ionization \\
\hline$\beta$ & $=$ & scale length \\
\hline$\Delta$ & $=$ & shock stand-off \\
\hline$\epsilon$ & $=$ & density ratio, emisivity, recombination energy \\
\hline$\theta$ & $=$ & angle from stagnation point \\
\hline$\lambda$ & $=$ & wavelength \\
\hline$\lambda ; \lambda_{\mathrm{D}}$ & $=$ & path length, Debye length \\
\hline$\rho$ & $=$ & mass density \\
\hline$\nu$ & $=$ & frequency \\
\hline$\sigma$ & $=$ & electrical conductivity \\
\hline$\tau$ & $=$ & collision time \\
\hline$\psi$ & $=$ & viscosity \\
\hline $\ln \Lambda$ & $=$ & Spitzer logarithm \\
\hline$w_{c}$ & $=$ & cyclotron frequency \\
\hline & & viii \\
\hline
\end{tabular}




\section{SUBSCRIPTS}

\begin{tabular}{|c|c|c|}
\hline a & $=$ & neutral atom \\
\hline $\mathrm{b}$ & $=$ & body \\
\hline B & $=$ & with magnetic field \\
\hline c & $=$ & calibration \\
\hline e & $=$ & electron \\
\hline $\mathbf{h}$ & $=$ & heavy particle \\
\hline $\mathbf{L}$ & $=$ & lateral \\
\hline $\mathbf{o}$ & $=$ & stagnation \\
\hline$\infty$ & $=$ & freestream \\
\hline $\mathbf{u}$ & $=$ & state \\
\hline $\mathbf{s}$ & $=$ & shock, source \\
\hline 1,2 & $=$ & spatial position \\
\hline$\nu$ & $=$ & frequency \\
\hline $\mathbf{n}$ & $=$ & excited state \\
\hline $\mathbf{w}$ & $=$ & wa11 \\
\hline 1 & $=$ & ion \\
\hline$t$ & $=$ & tank \\
\hline
\end{tabular}

\section{SUPERSCRIPTS}

$\begin{array}{ll}* \quad & \text { dimensionless quantity } \\ 0 & =\quad \text { stagnation point }\end{array}$




\section{LIST OF TABLES}

Table I: Literature Survey

Table II: Spectroscopic Information

Table III: Empirical Equations for the Data 
$\bar{L}-\cdots \cdot=$

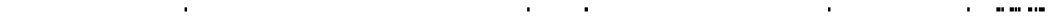




\section{LIST OF FIGURES}

1. Comparison of aerodynamic and magnetoaerodynamic flow

2. Schematic of facility

3A. Photograph of facllity showing operating console

3B. Photograph of facility showing spectroscopic apparatus

4. Photograph of pressure probe and vartous heads

5. Freestream property profiles

6. Interfor detall body and magnet

7. Photograph of bodies and magnets used in magnetoaerodynamic drag measurements

8. Photograph of drag measuring apparatus

9. Typical force calibration for drag tests

10. Sample magnetic field strengths

11. Field drop off with axial distance

12. Typical trace of total drag measurement. (The apparent difference ( $\sim \mathrm{sec}$ ) between the initial rises of both traces is due to mechanical crossover. Rises are actually simultaneous)

13. Alteration to total drag and components with magnetic field

14. Comparison of total drag alteration for two body shapes

15. Pressure distribution on body for two tap sizes

16. Pressure change at various angles as a function of magnetic field

17. Pressure distribution on body for several field strengths

18. Photograph of body in flow with and without magnetic field

19. Wilson's criterion for state equilibrium with free electrons

20. Schematic of spectroscopic experiment

21. Response of phototube

22. Spectral scans showing continuum and some lines measured

23. $4300 \AA$ line and underlying continuum 
24. $4510 \AA$ line with and without magnetic field

25. Typical Inversion of lateral scan

26. Sample Boltzmann Plot

27. Centerline electron temperature and body density with and without field

28. Radial profiles of electron temperature and number density in the stagnation region

29. Showing the photographic technique used to measure shock standoff alteration

30. Comparison of photographic and spectroscopic methods for measuring shock standoff

31. Relative Boltzmann plot at $0.65^{\prime \prime}$ axial position

32. Radial profiles of several spectral lines

33. Empirical relations for drag data

34. Comparison of total drag data and theory

35. Comparison of Lorentz drag data and theory

36. Comparison of theoretical and experimental pressure force measurement

37. Comparison of shock standoff data and theory

A1. Schematic of facility

A2. Operating characteristics of facility

A3. Schematic of drag measuring device

B1. Impact pressure probe -- Interior detail

B2. Typical impact pressure surveys

B3. Mass flux experiment -- detail 


\section{CHAPTER I}

\section{INTRODUCTION}

Entry of a hypersonic vehicle into the atmosphere is known to present severe problems caused by the shock-heated gas at the nose of the body. Not only is heat transfer to the body extreme but due to ionization in the gas, communications to the vehicle are difficult during part of the entry flight.

Accompanying the interest in the problems of high speed entry is the proposal by Kantrowitz (Ref. 1) and by Resler and Sears (Ref. 2) to utilize the interaction of the fleld of an onboard magnet with the ionized flow in the forward region of the vehicle. Consideration of this interaction has shown that a varlety of different effects will appear offering such possibilities as flight control based on the change in drag and ift on a vehicle with a magnetic field. Simultaneously, heat transfer to the body Is reduced.

Figure 1 depicts the changes in the hypersonic flow around a blunt body when the flow is ionized and a magnetic field is applied. Here the body is a simple hemisphere at zero angle of attack to the flow. The magnet is presumed dipole-1ike, with the axis aligned with the flow. Due to the flow of charged particles with velocity $\underline{V}$ across a magnetic field $\underline{B}$, an electric field and consequently a current $J$ is induced in the azimuthal direction in accordance with Ohm's Law. The interaction of this current with the magnetic field results in Lorentz force $\underline{F}$ on the fluid. It is seen that this force is given by $\underline{F}=\underline{J} \times \underline{B}=\sigma \underline{V} \times \underline{B} \times \underline{B}$ where $\sigma$ is the conductivity of the gas and the force is directed to oppose the flow. 
In a normal aerodynamic flow of this nature, the shock wave which forms In front of the blunt body is detached to permit passage of the flow around the body as dictated by continuity. Since the flow is slowed when the magnetic field is applied, the detachment distance must therefore increase. Likewise the viscous drag and heat transfer on the body must change since these quantities depend on the gradient of velocity normal to the body which is altered. A component of the force on the fluid lies along the direction of the freestream flow. This force partially supports the flow in the shock layers so that the pressure force on the body is relleved. Simultaneously the reaction to this component acts directly on the magnet, thus the drag force on the body is increased by this amount.

In this explanation we have avoided the Hall effect by taking Ohm's Law in its simplest form, i.e., we have ignored the reduction in conductivity in the direction of the electric field and the introduction of transverse currents, both due to the application of a magnetic field. A reduction in conductivity in the azimuthal direction will lead to a reduction in any advantage gained by the application of a magnetic field. Furthermore, the additional Hall currents also interact with the magnetic field to produce a force in the azimuthal direction on the fluid and a torque on the magnet.

We have only discussed a small part of the phenomena of magnetoaerodynamics. An elementary consideration of magnetoaerodynamic lift could proceed in a similar manner with the body or magnet or. both taken at an angle of attack to the flow (see Ref. 3). In a practical flight situation a vehicle entering the earth's atmosphere passes through a regime of rarefied flow. Here electric forces on the body and their alteration by a magnetic field 
are known to be important. For a discussion of these phenomena the reader 18 referred to Ref. 4.

This study is a laboratory investigation of the magnetogasdynamic flow around a blunt body at supersonic speeds. The fundamental aspects of magnetoaerodynamics are examined for a simple case. We restrict the study to an axisymmetric conducting body with either a hemispherical or flat end nose, at zero angle of attack. The axis of the solenoidal magnet is aligned with the flow. In addition to these restrictions, we concentrate on a single continuum flow condition and examine in detail the magnetoaerodynamic drag on the body and the alteration to the shock layer. Under these restrictions we have precluded an investigation of magnetoaerodynamic lift or electric forces on the body. Furthermore, in the laboratory it is expected that interaction of the flow and magnetic field leaves the magnetic field undistorted (Ref. 5). In other words, the appropriate parameter, the magnetic Reynolds number (to be discussed and evaluated 1ater) is so small that distortion of the magnetic field Iines is negligible. While we are primarily concerned with a laboratory problem, qualitative differences with a flight situation will be noted as they occux.

The rest of this chapter contains a review of the literature pertalning to blunt body magnetoaerodynamic flows (summarized in Table I). Following this the equations of magnetogasdynamics are stated and a preliminary analysis of the problem is made. Chapter II presents a background for the laboratory investigation and Chapter III concerns the facility in which the experiments are to be performed. The experiments pertaining to magnetoaerodynamic drag are reported in Chapter IV. Similarily, Chapter V reports 
the detailed examination of the flow fleld and measurement of magnetoaerodynamic shock standoff distance by optical methods. The results of Chapters IV and $V$ are further discussed and comparison is made to theory in Chapter VI. Chapter VII contains a summary of the Investigation and recommendations for future research.

\subsection{Literature Survey}

In 1958, following the inftial suggestions of the possible uses of magnetogasdynamics during entry, Bush (Ref.6) treated the problem of magnetoaerodynamic hypersonic flow around a blunt body. The analysis is confined to the stagnation region and assumes an inviscid flow of constant density and constant conductivity behind the shock. Specifying the magnetic fleld at the shock and substitution of suitable functions for the fleld and velocity permits reduction of the problem to a point where it may be handled numerically. Both the case of small and of vanishing magnetic Reynolds numbers are treated. Results are presented in terms of the magnetic interaction parameter defined using the magnetic field strength at the shock and the radius of the shock as the characteristic dimension. Thus, $s_{s}=\frac{\sigma_{1} R_{s} B_{s}}{P_{\infty} V_{\infty}}$ where $\sigma_{1}$ is the conductivity behind the shock and the subscript $\infty$ denotes freestream conditions. The results show an increase in shock standoff with field strength and a decrease in pressure off axis. The pressure is calculated from the relation $\frac{P-P_{0}}{1 / 2 P_{\infty} V_{\infty}^{2}}=Q_{\theta}^{2}$, which is obtained by integrating the $\theta$ momentum equation. Here $P$ is the pressure on the body surface, $P_{0}$ the stagnation pressure, 
$1 / 2 P_{\infty} v_{\infty}^{2}$, the freestream dynamic pressure, and $\mathcal{P}$, the so-called pressure relief function which is calculated numerically.

At the same time Kemp (Ref. 7) presented a similar theory with similar assumptions but further restricted his analysis to $R_{e m}=0$ and small interaction parameter. His Initial results show a pressure increase but the analysis was later questioned by Freeman (Ref. 8). [See also Author's Reply (Ref. 9).] The conclusion of this work was that the pressure was in fact unaffected for small interaction.

The first experimental work, due to Zeimer (Ref. 10) investigated the alteration to the shock standoff distance with field. This investigation was conducted in an electromagnetic shock tube facility using a model with a transient magnetic field ylelding results over a substantial range of Interaction. Reasonable agreement for shock standoff distance was obtained when compared to Bush's results.

Wu (Ref. 11) considered constant viscosity, density and conductivity model in an analysis similar to Bush. His results show that the effect of viscosity is to decrease the magnitude of the alteration to shock standoff with field. Lykoudis (Ref. 12) treated a flow model similar to that of Bush but assumed the Newtonian-Busemann pressure relationship in his solution. This permits him to obtain an analytical solution for a smal1 range of interaction because the pressure distribution (on the body, in particular) is specified. He also assumes a constant radial magnetic field which is unrealistic physically, inasmuch as a practical magnet contained in the body would have a decreasing radial field. To account for this fact the author has suggested a mean value for the field In Ref. 13. 
Pai and Kornowski (Ref. 14) have performed the same analysis as Bush except that the magnetic field specified at the shock is assumed to vary as some power of the radius $\left(r^{-3}\right.$ for a dipole). The solutions presented for different values of the exponent show the same qualitative result with regard to the pressure relief as Bush's solution.

In Ref. 15 Levy has treated a simplified two-dimensional magnetoaerodynamic flow over a cylinder. An attempt is made to account for the Hall effect by reducing the scalar component of conductivity in the direction of $\underline{V} \times \underline{B}$ as follows: $\sigma_{\text {red }}=\frac{\sigma}{1+C_{H}^{2}}$ where $C_{H}$ is the Hall coefficient.

This, of course, does not account for the transverse Hall currents. The Importance of the Hall effect in reducing the interaction is emphasized by his simple example.

Chuskin (Ref. 16) has treated the hypersonic flow around a magnetized sphere and cylinder. His model is inviscid but includes compressibility and the electrical conductivity is given by a specified temperature dependent law. The problem is formulated by the method of integral relations and then solved by numerical techniques. In formulating the problem in terms of integral relations (the method of Belotserkovskii), Chuskin obtains an advantage over the formulation of Bush, in that the analysis is not limited to small angle approximations. Variations in physical properties behind the shock may be easily introduced. Results obtained show that as is the case when viscosity is included, allowing the conductivity to vary causes a decrease in effect. His results are shown only for relatively small interaction and in the limit of $\mathrm{M}_{\infty}=\infty$. While the 
standoff Increases with field so does the pressure off axis on the body which appears to be contrary to Bush's results. Heat transfer is also foind to increase.

Smith, Schwimmer and Wu extended earlier works (Refs. 11 and 18), which included viscosity in an analysis similar in method to that of Bush. This work was questioned by Porter and Cambel (Ref. 19) on the inclusion of viscosity and certain details concerning the evaluation of boundary conditions. Correction of these errors resulted in agreement with previous works (Ref. 20).

A group of papers (Refs. 21, 22, 23) deal with the opposite extreme from that discussed in previous works. Here the magnetic interaction is taken as dominant, the shape and in fact the presence of the body make no difference. Levy and Petscheck (Ref. 21) first analyzed the case of two-dimensional hypersonic flow over a straight current carrying conductor. The magnetic Reynolds number was first assumed small due to small conductivity in a preliminary analysis. Subsequent1y, the high Reynolds number - high conductivity case was treated. The density was taken equal to pressure as the thermodynamic relationship. The results show an increase in shock standoff with increasing wire current. At high currents the shock layer was found to lift completely off the wire. Experiments (Ref. 22) were performed in a Joule-heated, helium-driven gas, diaphram shock tube. The results indicate reasonable agreement with the theory for shock position, although the photographs presented do not indicate a no-flow region adjacent to the body, a feature of the theoretical analysis. 
A later and more detailed analysis of the corresponding three-dimensional problem was made by Levy et al., (Ref. 23). The existence of a magnetically dominated flow for this problem assuming negligibiy sma11 magnetic Reynolds numbers is shown. The effects of variable properties In the shock layer are treated although the Hall effect is not. The analysis shows that the shock layer occurs at some position ahead of the magnetic source so as to balance the magnetic forces and the momentum change of the incoming flow. Behind the shock layer and in the stagnation region a region of "slow flow" forms which rejoins the shock layer outside the stagnation region. These results were obtained in the limit of $\epsilon^{1 / 4}$ small where $\epsilon$ is the density ratio across the shock $\frac{P_{\infty}}{P_{s}}$. In considering a problem in which this restriction may not be realistic, it is concluded that similar phenomena should result but less distinctly. It 18 also commented that the unexplained differences in the results of Refs. 6 and 7 for the pressure terms are resolved by considering the location of the source relative to the shock and shock layer center of curvature, although these statements appear to be based solely on the original papers and not on subsequent work.

Ericson and Macuilatis (Ref. 3) have applied an analysis similar to Bush to the problem of magnetoaerodynamic lift. While primarily concerned with the application of this phenomena to flight, several parts of the analysis are pertinent to this discussion. In choosing the "volume of Interaction," the volume of flow which contributes substantially to magnetoaerodynamic forces, these authors take $\theta_{c}=60^{\circ}$ (the angle at which the calculations are terminated) to account for the outward movement of the sonic line when the flow is slowed. Although the Hall effect 
1s not genera11y treated they utilize a reduced conductivity simflar to that of Ref. 15 in evaluating the magnetic interaction parameter. In later work (Ref. 24) the analys is is reviewed and the magnetically dominated flow concept is discussed.

In an extensive investigation, Porter (Ref. 25) has calculated magnetoaerodynamic flow around a hemispherically capped cylinder. Using a constant property model and 1ike Bush, specifying the magnetic field at the shock, he was able to numerically obtain solution for the flow field, the shock standoff, the drag coefficient and the pressure distribution. The Hall effect, the effect of viscosity and the effect of finite magnetic Reynolds numbers are taken into account in separate solutions. To obtain the drag coefficients it was necessary to specify a volume of interaction outside of which it is assumed that no interaction takes place. His results for the shock standoff reduce to those of Bush for no Hall effect or viscosity. Likewise, the pressure is found to be decreased for this case, but it is significant to note that for a constant value of the interaction parameter the pressure relief is seen to lessen and finally become a pressure increase as the Hall effect is increased. Although he was not able to handle viscosity and the Hall effect simultaneously, it is suggested that the viscous solution merges with the invscid solution for large interaction $(S=50)$ : Thus, an extended composite theory is offered to account for these effects together.

Seeman and Cambel (Ref. 26) have reported an experimental investigation of total magnetoaerodynamic drag and shock-standoff for a hemispherlcally capped cylinder (conducting and nonconducting materials were both used) with a self-contained electromagnet. Tests were conducted in 
a continuous arc-heated facility. Increases in drag and shock-standoff distance were measured for interaction parameter (defined at the shock) values of the order of $s_{s}=1$. These results are presented in terms of applicable dimensionless parameters and the standoff measurements are compared to the theories of Bush and Lykoudis. Agreement with these theories is found by multiplying the magnetic interaction parameter by the shock density ratio, $\epsilon$. Drag increases of the order of $10 \%$ were obtained and a qualitative evaluation of the drag data was made. More recently $R$. Nowak, et al. (Ref. 27) have investigated the same problem as Ref. 26, but extending the conditions of the experiment to a wider range of pressure, density and interaction. Total drag measurements for several flow conditions are brought together with the parameter $S \in$. The results show agreement with the theoretical predictions of Ref. 25, thus emphasizing the influence of the viscous and Hall effects in reducing total drag and shock standoff distances. The results of Refs. 25, 26, and 27 have recently been discussed further by Cambel (Ref. 28) along with a review of the entire problem of blunt body magnetoaerodynamics.

\subsection{Description of Magnetoaerodynamic Flow}

In reviewing the literature we have seen that the gross qualitative aspects of this problem, the change in shock standoff and total drag on the body, with increasing magnetic field are reasonably well understood in theory and are confirmed by experimental observation. The qualitative features of the alteration to the pressure distribution, however, do not appear to be in agreement in various analyses and no experimental measurements of the pressure distribution have been made. 
We have also seen that most analyses up to the present deal with highly idealized models due to mathematical complexities. The limitations of theoretical treatments may be grouped into three general areas: the initial assumptions made; the expected validity of the results; and the ability to handle several complicating factors simultaneously. A major simplifying assumption commonly made is constant properties in the shock layer. While the reasonableness of this assumption depends somewhat on the physical example to which it is applied, one could immediately question the assumption of constant conductivity as Chuskin (Ref. 16) has done. This assumption also requires the concept of a "volume of interaction" in order to calculate the force on the magnet or the pressure force (Refs. 6 and 25).

The usefulness of theories which are restricted to small interaction is limited to predicting trends and ordering the magnitude of effects. Even when the interaction is taken as arbitrary (for example, Ref. 25) the validity is often restricted to stagnation point analysis, with the accuracy decreasing as the region is extended. Of the combination of effects which may be present those of viscosity and Hall effect and possibily compressibility have not been treated together, although as we have noted earlier, an approximate theory for considering the Hall effect and viscosity together has been presented in Ref. 25. Elementary calculations have shown that these effects will occur together in the laboratory and some flight regimes (Ref. 27). We are therefore limited to the knowledge that the inclusion of dissipative mechanisms generally leads to a decrease in magnetoaerodynamic effect. 
To discuss the analytical and physical aspects of the problem we require a realistic model, yet one not so complicated as to be unwieldy. Bearing in mind that many of the theoretical limitations previously discussed are those features which also make the problem manageable, we write the following equations governing the problem and make some preliminary assumptions:

Time independent Maxwel1 equations:

$$
\begin{aligned}
& \nabla \cdot \underline{B}=0 \\
& \nabla \cdot \underline{E}=\frac{\rho_{e}}{\epsilon_{0}}\left(\rho_{e}=\text { excess charge density, } \varepsilon_{0}=\right.\text { dielectric constant) } \\
& \nabla \times \underline{E}=0 \\
& \nabla \times \underline{B}=\mu_{O} J \begin{array}{c}
(\text { the permeability of the plasma equals } \\
\text { that of free space) }
\end{array}
\end{aligned}
$$

Conservation of Charge:

$$
\nabla \cdot \underline{J}=0
$$

Ohm's Law:

In simple scalar form, $\quad \underline{J}=\sigma(\underline{E}+\underline{V} \times \underline{B})$

Including the Hall effect Ohm's Law may be written as a vector equation (Ref. 15),

$$
\underline{J}=\sigma(\underline{E}+\underline{V} \times \underline{B})+C_{H}(\underline{J} \times \underline{B})
$$

where $\sigma$ is the scalar conductivity. An alternate (but not equivalent) expression takes $\mathbb{g}$ as the conductivity tensor (Ref. 29). Thus,

$$
\underline{J}=\underline{g} \cdot(\underline{E}+\underline{V} \times \underline{B})
$$


These expressions assume the momentum uncoupling between the ions and atoms (ion slip) to be negligible.

The applicable magnetogasdynamic equations may be written as follows for steady flow (Ref. 28).

Continuity:

$$
\nabla \cdot \underline{V}=0 \text { (for incompressible flow) }
$$

Momentum:

$$
\rho \frac{\nabla \underline{v}^{2}}{2}-\rho \underline{V} \times \nabla \times \underline{v}=-\nabla p+r \nabla^{2} v+\underline{J} \times \underline{B},
$$

taking $\pi \nabla^{2} \underline{V}$ for the divergence of the stress tensor, $\nabla \cdot T$ (Ref. 30). It is well known that if the constant property assumption is made then it is unnecessary to write an energy equation. For the present we shall also make this assumption. However, we recognize that a close examination of the flow is likely to necessitate an energy equation and while a global energy equation may be written as for continuity and momentum, it will prove most practical to use the species energy equation, in particular for the electrons, since properties such as conductivity and radiation from the flow are governed most strongly by the energy of the electrons. The electron energy equation will be considered extensively in Chapter V.

We now discuss the scaling of the momentum equation, specifying that the magnetic field is that of a dipole

$$
\underset{\sim}{\mathrm{B}}=\mathrm{B}_{0} \cos \theta \widetilde{\mathrm{r}}+\frac{\mathrm{B}_{0}}{2} \sin \theta \tilde{\theta}
$$

and remains undistorted. This simplification is in accordance with 
Ref. 5, if the nondimensional magnetic Reynolds number

$$
\operatorname{Rem}=\text { en } \sigma V_{\infty}<0.1
$$

where the product $\epsilon V_{\infty}$ represents the velocity in the shock layer.

To nondimensionalize Eq. (1.7), the velocity and density are

referenced to their freestream values $\rho_{\infty}, V_{\infty}$ and the pressure to $1 / 2 \rho_{\infty} V_{\infty}^{2}$. The magnetic field is referenced to $B_{0}$, the stagnation point value and the radius $r$ to the body radius $R_{b}$. Then $\mathrm{Eq}$. (1.7) becomes (taking $p^{*}, p^{*}, V^{*}$ and $B^{*}$ as non-dimensional quantities)

$\frac{\rho^{*} \nabla \underline{V^{*}}}{2}-\rho^{*}\left(\underline{V}^{*} \times \underline{\nabla} \times \underline{V^{*}}\right)=-\frac{\nabla p^{*}}{2}+\frac{\eta}{\rho_{\infty} \nabla_{\infty} R} \nabla^{2} \underline{V^{*}}+\frac{\sigma \mathrm{R}_{\mathrm{b}} \mathrm{B}_{0}^{2}}{\rho_{\infty} \mathrm{V}_{\infty}} \mathrm{J} * \times \mathrm{B}^{*}$

We recognize

$$
\begin{aligned}
& \operatorname{Re} \text { (Reynolds Number) }=\frac{\rho_{\infty} V_{\infty} R}{\eta} \\
& S \text { (Interaction Parameter) }=\frac{\sigma R_{b} B_{0}^{2}}{\rho_{\infty} V_{\infty}}
\end{aligned}
$$

which together with the non-dimensional Hall coefficient from Eq. (1.4)

$$
c_{H}=\frac{e T_{e i}}{m_{e}} B_{o}
$$

are the important non-dimensional parameters of the problem.

The interaction parameter is often interpreted as the ratio of Lorentz force $=\sigma B_{0}{ }^{2} L V$ to dynamic force $=\rho V^{2}$. The Lorentz force should be evaluated in the shock layer however, and the dynamic pressure is evaluated in the freestream. Observing that we may divide equation (1.10) 
by $p^{*}=\frac{\rho}{P_{\infty}}$ the non-dimensional interaction parameter behind the shock

$$
S e=\frac{\epsilon V_{\infty} \sigma R_{b} B_{o}^{2}}{\rho V_{\infty}^{2}}
$$

since $\epsilon=\frac{P_{\infty}}{P}=\rho$ * across the shock. We may then interpret $\epsilon V_{\infty}$ as the velocity in the shock layer. Thus, the parameter $€$ is also important in the problem and $S e$ rather than $S$ is the more natural parameter.

At this point it is we11 to review the problem, outline and summarize the constraints already made. We have limited the investigation to an axisymetric conducting body containing an electromagnet with both the magnet axis and body axis aligned with the flow. A sufficiently low magnetic Reynolds number has been assumed so that the magnetic field which is taken to be a dipole remains undistorted. This parameter remains to be evaluated for laboratory conditions. For the present a physical model based on constant properties has been adopted since this appears to have permitted the most extensive theoretical treatments. The validity of this assumption is left open for later review. 


\section{CHAPTER II}

\section{CONSIDERATIONS IN THE MEASUREMENT OF DRAG AND SHOCK STANDOFF}

The preceding 1iterature survey indicates the lack of experimental work pertaining to magnetoaerodynamics in contrast to a number of theoretical treatments. The purpose of this chapter is to discuss laboratory investigation of this problem. First, quantities which can be measured are formulated from the flow description made in Chapter I. Previous techniques are reviewed with emphasis on technique and the limitations of these works is used to provide direction for this investigation. Finally, preliminary experiments made in this investigation are discussed and the shortcomings of these are noted.

\subsection{The Components of Magnetoaerodynamic Drag}

The total drag on a blunt body in magnetoaerodynamic flow is composed of the pressure force on the frontal surface, the pressure force on the base, the viscous force on the body and the reaction force on the magnet. In addition to measurements of the total drag, it is desirable to examine independently as many of these components as possible, not only for Individual comparison to theory, but also to understand more completely the composition of the total drag.

Furthermore, the various drags are integrated quantities. If measurement of the pressure distribution, which is a local quantity, is made then additional information concerning magnetoaerodynamic interaction will be made available.

The coefficients of magnetoaerodynamic drag have been formulated by Porter (Ref. 25) where the coefficient of a particular component of drag 


\section{Is given as}

$$
C_{D \text { comp }}=\frac{\text { Component Force }}{1 / 2 P_{\infty} V_{\infty} A_{\text {prof }}}
$$

where $A_{\text {proj }}=\pi R_{b}{ }^{2}$, the projected area of the body. We shall use a similar development to derive expressions for the component forces.

Consider the force on the magnet first. This is given as the reaction to the component parallel to the freestream of the Lorentz force on the fluid and has magnitude

$$
L_{\mathrm{F}}=\int_{\mathrm{vol}}(\underline{\mathrm{J}} \times \underline{\mathrm{B}})|| \mathrm{dvol}
$$

The precise choice of the volume over which this integration takes place Is a matter of some concern as we have pointed out. To review, theoretical treatments, taking a constant property approach and considering the freestream to be nonconducting, take this volume to be a spherical cap on the nose of the body, the thickness of the shock standoff. The calculation is then terminated at some angle $\theta_{c}$ to account for the fact that properties such as electrical conductivity are not constant and the influence of magnetic interaction is finite in extent. Values of $\theta_{c}$ chosen range from $\theta_{c}=30^{\circ}$ to $\theta_{c}=60^{\circ}$. For the present we will assume $\theta_{c}=45^{\circ}$ (following Ref. 25). While $\theta_{c}$ is not a physical quantity, calculation of such quantities as the force on the magnet are sensitive to the value of $\theta_{c}$ chosen. Thus the equation becomes

$L=\int_{R_{b}}^{R_{s}} \int_{0}^{\theta} c\left[-\left(V_{r} B_{\theta}^{2}-v_{\theta} B_{r} B_{\theta}\right) \cos \theta+\left(B_{r} B_{\theta} V_{r}-V_{\theta}{ }_{r}^{2}\right) \sin \theta\right] r^{2} \sin \theta d r d \theta$ 
The viscous drag is proportional to the normal gradient of the flow cangential to the body. For the nose region the component of this force parallel to the freestream flow is given by

$$
v_{f}=2 \eta_{1} \int_{0}^{\pi / 2} \frac{\partial v_{\theta}}{\partial r} \sin ^{2} \theta d \theta
$$

where $\eta_{1}$ is the viscosity in the region behind the shock. The shear forces on the after body may be similarly formulated.

Since the pressure distribution is discussed extensively in later sections, the formulation and theoretical treatment will be examined in detai1. The $\theta$ component of the momentum Eq. (1.7) is

$$
\begin{aligned}
& \frac{1}{r} \frac{\partial V_{r}^{2}}{\partial \theta}+\frac{1}{r} \frac{\partial V_{\theta}^{2}}{\partial \theta}+\frac{1}{r} \frac{\partial V_{\Phi}}{\partial \theta}+\frac{1}{\rho r} \frac{\partial P}{\partial \theta}= \\
& \frac{V_{\Phi}}{r \sin \theta} \frac{\partial}{\partial \theta}\left(\sin \theta U_{\Phi}\right)-\frac{V_{r}}{r} \frac{\partial}{\partial r}\left(r V_{\theta}\right)-\frac{V_{r}}{r} \frac{\partial V_{r}}{\partial \theta}+ \\
& \frac{\eta}{\rho}\left[\frac{1}{r^{2}} \frac{\partial}{\partial r}\left(r^{2} \frac{\partial V_{\theta}}{\partial r}\right)+\frac{1}{r^{2} \sin \theta} \frac{\partial}{\partial \theta}\left(\sin \theta \frac{\partial V_{\theta}}{\partial \theta}\right)-\frac{V_{\theta}}{r^{2} \sin ^{2} \theta}+\frac{2}{r^{2}} \frac{\partial V_{r}}{\partial \theta}\right] \\
& +\frac{1}{\rho} J_{\Phi} B_{0} \cos \theta
\end{aligned}
$$

Using suitable functional forms for the velocities and currents the authors of Ref. 6 and Ref. 25 find that this equation, which contains the pressure, may be directly integrated with respect to $\theta$ under the approximation for the stagnation region that 


$$
\begin{aligned}
& \cos ^{2} \theta \cong 1-\theta^{2} \\
& \sin ^{2} \theta \cong \theta^{2}
\end{aligned}
$$

These particular functional forms are given by (Ref. 25)

$$
\begin{aligned}
& v_{x}=2 \epsilon v_{\infty} \frac{F(x)}{x^{2}} \cos \theta \\
& v_{\theta}=-\epsilon v_{\infty} \frac{F^{\prime}(x)}{x} \sin \theta \\
& V_{\Phi}=\epsilon v_{\infty} \frac{G(x)}{x} \sin \theta \\
& J_{\Phi}=\epsilon v_{\infty} \sigma_{1} B_{0} \frac{L(x)}{x} \sin \theta
\end{aligned}
$$

where $x=\frac{r}{R_{b}}$, This integration results in

$$
P-P_{0}=\frac{1}{2} \theta^{2} \Theta \rho_{\infty} \nabla_{\infty}^{2}
$$

where $\Theta$ the pressure relief parameter on the body surface $(x=1)$ is given by

$$
\Theta=\epsilon\left[F^{\prime}(1)^{2}-G(1)+\frac{F^{\prime \prime \prime}(1)}{R_{e}}-S L_{(1)}{ }^{\prime}\right]
$$

To determine this the pressure distribution on the body, the functions $F^{\prime}$, G, $L^{\prime}$ must be evaluated on the body and suitable boundary conditions applied. By application of the governing equations further reduction of Eq. (2.9) is possible. We find:

(inviscid, $\operatorname{Rem}=0) \Theta=\left.\epsilon\left\{F^{\prime 2}+G^{2}-S F^{\prime}-C_{H^{\prime}} F^{\prime}\right\}\right|_{\text {body }}$ 
(viscous, $\operatorname{Rem}=0) \quad=\left.\in\left\{F^{\prime 2}+F^{\prime \prime \prime} / \operatorname{Re}-25 F^{\prime}+\frac{C_{H}}{\operatorname{Re}} G^{\prime \prime}\right\}\right|_{\text {body }}$

Both expressions account for the Ha1l effect and involve only velocities.

$Q$ may be determined for the inviscid case (the first of Eq. $(2.10)$ ) by substituting numerically calculated values of $F^{\prime}$ and $G$. No such solution exists for the second of Eq. (2.10), since this includes both Hall and viscous effects. Bush and Porter have determined the pressure relief. parameter $\odot$ for the inviscid case and Porter has extended these results to the Inviscid case with Hall effect as well as the viscous case without Hall effect. The pressure force is then determined by integrating the pressure distribution over the nose area of the body. Thus,

$$
P_{f}=2 \int_{0}^{\pi / 2} P(\theta) \pi R_{b}^{2} \sin \theta \cos \theta d \theta
$$

The magnitude of the base pressure may be calculated using the pressure in this region integrated over the area.

$$
B_{f}=P_{\text {base }} A_{\text {base }}
$$

Since the calculated value of the base pressure is small (Chapter IV) we assume the alteration to it may be neglected and simply correct for the no field base pressure in formulating the total drag. The total drag is then the sum of these forces:

$$
\text { Total Drag }=\mathrm{V}_{\mathrm{f}}+\mathrm{P}_{\mathrm{f}}+\mathrm{B}_{\mathrm{f}}+\mathrm{L}_{\mathrm{f}}
$$

\subsection{Previous Experimental Work}

Seeman (Ref. 31) has measured the total magnetoaerodynamic drag on a 
hemisphere capped body in a continuous arc-heated low density facility. To accomplish the measurement he used a strain gauge system fixed directly to the supporting sting. To provide a magnetic field he used a copper wire coil wound on an iron core giving a maximum field of about 2,000 gauss. This fleld strength limited the maximum interaction parameter at the shock to about 1 and prevented investigation of the Hall effect. Based on theoretical considerations of this problem (Ref. 25) we would further conclude that neither the pressure force nor the viscous forces were substantially altered when the magnet was turned on so that the total drag alteration was due to the force on the magnet. Seeman made preliminary design estimates of this force by assuming no Hall effect and using the simple form of $\mathrm{Ohm}^{\prime} \mathrm{s}$ Law in Eq. (2.3). The total force on the body was calculated by a conventional aerodynamic model and the heating rate from the high temperature plasma to the body was estimated to plan for cooling the model.

Both Seeman and Zeimer (Ref. 31 and 10) measured the alteration to shock standoff distance by direct measurement of photographs. Seeman and Cambel have commented on the discrepancies involved in these measurements due to the diffuse nature of the shock front (Ref. 26).

In summary, we expect technical problems similar to those of Ref. 31 since our experiment is very similar. For preliminary design estimates of drag and heating rates the values given in Ref. 31 will be taken. In considering these experiments we would attempt to extend the range of interaction and to find a more acceptable method for investigating the shock standoff alteration. 


\subsection{Preliminary Experiments}

The arc-heated low density facility constructed for this investigation is fully described and analyzed in Chapter III and Appendix A. For purposes of the present discussion we note that this is a continuous facility as contrasted to typical shock tube facilities which have very short duration test times. Thus, the planning of experiments concerning magnetoaerodynamic drag need not be limited by the operation of the facility but rather by the high heating rates encountered in this type of facility as well as limitations on the mode1 itself. In particular the magnet test time may be limited by Ohmic heating of the coll. The planning of experimental equipment must also take into account the low pressure and electrically noisy environment found in these types of facilities.

With the background provided by Ref. 31 a preliminary drag measuring system using semiconductor strain gauges was constructed and tested. This was found to be unsatisfactory due to hysterisis in the mechanical system. Instead a linear differential transformer was found to give good results once adequate shielding against the high enthalpy flow had been installed. Two complete mechanical systems were designed and constructed, and this final design will be described fully in Chapter I.V. The only important difference between these two designs is that efforts were taken in the second system to keep the sting support small in comparison to the body diameter to minimize interference with the base region.

In considering Seeman's experiment it seemed to be most important to increase the magnetic field strength in order to increase interaction. An obvious advantage is that the magnetic field enters into the interaction 
parameter as the second power in Eq. (1.12).

The use of superconducting magnets was considered but these were found unsatisfactory after design estimates were made because the thickness of the Dewar flask (for cooling) surrounding the magnet lowered the field strength to less than that attainable by conventional magnets. Through experimentation it was found that the field strengths of conventional magnets were increased by the choice of better core material and careful positioning of the magnet with respect to the nose of the model. Tests also Indicated that for the duration of the drag tests $(\sim 2 \mathrm{sec})$ the wire could withstand a considerable power overload. Final design and testing of these magnets is also described in Chapter IV.

Preliminary drag measurements were conducted using a three inch diameter model positioned at $121 / 2^{\prime \prime}$ from the nozzel exit. While substantial increases in drag could be measured, the flow was unsteady at this position giving rise to scatter in the data due to mechanical noise. Also it was decided that the model was too large for the flow. Experiments were conducted to determine whether these problems could be corrected by varying the flow conditions either by changing the nozzel configuration or varying the test conditions. Although some improvement was noted, it was found to be much better to move the model to 4" from the nozze1 and use a $11 / 2^{\prime \prime}$ body diameter.

Total drag experiments using a $11 / 2^{\prime \prime}$ diameter body at this position over the range of flow conditions available in the facility. This initial investigation is described in Ref. 27.

During this work it was found that the flow conditions available in the facility were limited either by the facility itself or by the fact 
that at an avallable condition the magnetic interaction was too small to glve substantial results. In summary, this preliminary work served to establish the conditions of the flow and facility under which experiments could be conducted and indicated directions for the design of final equipment. 
CHAPTER III

THE EXPERIMENTAL FACILITY AND EVALUATION OF FLOW CONDITIONS

The purpose of this chapter is to present background material for the experiments and discussions in subsequent chapters. The facility has already been mentioned briefly and is the subject of the first section of this chapter. The facility chosen for this investigation was an archeated, 1ow-density plasma wind tunnel, similar in nature to the type of facilities used by other investigators in experiments requiring high enthalpy, hypersonic flows and reasonably long test times (see for example, Refs. 32 and 33).

Analysis of the flow conditions in this facility is required next and serves two purposes. Through diagnostic measurement of flow conditions the nondimensional parameters appropriate to the experimental problem may be evaluated, the most important of these being the interaction parameter $S$, the Hall coefficient $C_{H}$ and $\epsilon$, the density ratio across the shock. Furthermore, the conditions of the flow where tests are to be conducted may be determined. This includes not only measurements of flow uniformity but also calculation of parameters such as viscosity and determination of the chemical state of the flow.

The final section of this chapter contains a discussion of the consistency of the data and calculated quantities and an evaluation of the error involved in determining the important nondimensiona1 parameters.

\subsection{The Experimental Facility}

The Gas Dynamics hyperthermal facility consists of a commercial direct current arc-heater, which exhausts into a $5 \times 11.5 \mathrm{ft}$. cylindrical 
vacuum tank. The expansion of the gas is through a conical nozzle $11 / 2^{\prime \prime}$ diameter exit with a $9 / 32^{\prime \prime}$ diameter throat and a half-angle of $15^{\circ}$.

The vacuum tank is continuously maintained at a low pressure by a 5,000 cfm impeller blower which is backed by a $460 \mathrm{cfm}$ rotary vacuum pump. The pressure is read by an "Alphatron" Gauge. Inputs for water and electricity are through the sides of the tank. The entire facility is controlled from an operating console and the flow is visible from this station through a viewing port. The facility is shown pictorially in Figure 2 and photographically in Figure 3. A more complete description of the facility is contained in Appendix A, along with specifications for the various equipment mentioned here.

Auxiliary equipment for use in this facility includes an overhead table which, controlled from the console, can be moved in three dimensions. This is used to support both the diagnostic devices and the models under test. Position of the table is read by cam operated microswitches and indicated at the console. Also available for the facility is a fast-acting heat shield which when in position deflects the flow to the side and allows a model or probe to cool. This is also controlled externally. Power for the arc is taken from the common laboratory power supply consisting of four rectifiers, commercial welders. The output may be filtered if required.

The external variables were mass flow and power which is measured by reading the voltage across torch and current through it. Heating losses were determined by measuring the flow rate of the cooling water and the temperature rise by thermocouples. The pressure in the stagnation chamber 
and arc chambers were variously read with mercury manometers and commercial pressure gauges.

\subsection{Choice of Flow Condition}

For experimentation we require a uniform continuum flow in which it is possible to produce high interaction. Data on the operation of the facility is presented in Appendix A. The operation of this facility is restricted to what may be characterized as a viscous to merged layer regime due to the avallable pumping capacity. Furthermore, it is difficult to change any of the external variables of the facility without substantially affecting all the flow quantities. (Cf. Figure A2 which shows some operating parameters of the facility plotted against mass flow for a constant arc current. For example it is seen that the mass flow cannot be changed without simultaneously changing the ambient pressure and the power input.) Over the limited range of variation where the flow is continuum, viscosity is always important.

We now consider the flow uniformity and interaction. The maximum size of the model is dictated by the maximum flow size. The model may be made smaller but this decreases the interaction parameter not only since $R_{b}$ decreases but also because the maximum stagnation field strength decreases. This is more important since the interaction depends on $\mathrm{B}_{\mathrm{O}}^{2}$. The model size selected on these considerations was $R_{b}=3 / 4^{\prime \prime}$. Then the conditions which seemed to give the most uniform, high conductivity flow were selected as the single operating conditions. (Some samples of radial pressure profiles at other conditions are shown in Figure B-2. For example, it is seen that moving the axial position of the pressure probe while 
holding the flow rate constant results in a more nonuniform flow. Changing the flow rate at the running position also increases the nonuniformity,) In addition, only argon gas was used, being attractive due to the low ionization potential, chemical inertness and large amount of data available for this gas.

It is realized that chosing a single flow condition is a severe limitation on the experiment. However, we emphasize that if different running conditions and model sizes were chosen new problems in relating results would be introduced. As was mentioned above, since no substantial change in such important parameters as Reynolds number can be made, no advantage is gained.

The running condition chosen for this investigation is most conveniently specified by stating the external quantities. These are:

$$
\begin{aligned}
I_{\text {Torch }} & =960 \text { amps } \\
V_{\text {Torch }} & =24 \text { volts } \\
\dot{\mathbf{m}} & =1.23 \mathrm{gm} / \mathrm{sec} \\
P_{\text {tank }} & =0.403 \mathrm{~mm} \\
P_{\text {stag }} & =.49 \mathrm{~atm} \\
\text { Power Loss } & =15.3 \mathrm{~K} . \mathrm{W} . \\
\text { Efficiency } & =33.5 \%
\end{aligned}
$$

Under these conditions the supersonic stream is self-luminous and Mach diamonds may clearly be seen. The flow is over expanded at the test station. 


\subsection{Diagnostic Measurements of Flow Conditions}

The research program of which this investigation is part includes a series of diagnostic measurements of flow conditions. While a number of techniques are available as a result of current research, we have concentrated on two probes of the type used in gas dynamics flow research, and spectroscopy. Other techniques were found to be either inconvenient because of the facility design or could not be used because of the flow conditions. Spectroscopic measurements of electron temperature and number density wil1 be fully discussed in Chapter V. For the present we w111 be concerned with the probe measurements of pressure and mass flux. Additional information and specifications may be found in Appendix B.

For the purposes of pressure measurements, a differential pressure transducer was mounted in a water-cooled chamber suspended from the overhead table. From this chamber a water-cooled sting extends down into the flow and different shaped heads may be soldered on the sting. The assembly is shown in Figure 4. This apparatus was used for a variety of impact, wedge, and static pressure measurements.

For impact pressure measurement, blunted cones of $15^{\circ}$ half-angle and a hemispherical shape were used with $1 / 16^{\prime \prime}$ and $1 / 8^{\prime \prime}$ taps. Static pressure measurements were made with a $1 / 2^{\prime \prime}$ diameter probe with taps normal to the surface located 10 diameters from the tip. Both $1 / 8^{\prime \prime}$ and $1 / 4^{\prime \prime}$ taps were used.

A mass flux probe was designed and constructed. The principle of this probe is that instead of forming a bow shock ahead of the probe the shock is swallowed and stands inside the probe. The fluid from the freestream 
which would normally flow around the probe is trapped in the probe. Thus, the mass flux into the probe represents the freestream mass flux. In order to accomplish this the probe was constructed in the following manner. A sharp-edged opening (both $1 / 8^{\prime \prime}$ and $1 / 4^{\prime \prime}$ diameter were used) is located at the tip of a $15^{\circ}$ cone. The diameter of the inside channel then enlarges and then exhausts into a large reservoir vacuum tank. This reservoir is maintalned at a pressure lower than the tank pressure before the test. The flow into the reservoir is initiated by opening a control valve. After a fixed period of time the pressure and temperature are measured at the reservoir, thus the mass flux can be deduced for choked flow.

The results of probe diagnostic measurements are as follows:

$$
\begin{aligned}
& P_{\text {impact }}=6.4 \mathrm{~mm} \\
& P_{\text {static }}=20 q \\
& P_{\infty} V_{\infty}=8.7 \times 10^{-3} \mathrm{gm} / \mathrm{cm}^{2} \mathrm{sec}
\end{aligned}
$$

Of these, the impact pressure appears to be most reliable. The static pressure is definitely lower than the ambient pressure of $400 \mu$, but the low Reynolds number makes the measurements difficult to interpret. The $\rho_{\infty} V_{\infty}$ measurements are also in question due to the difficulty of insuring that the shock is truly swallowed (choked flow) and the question of the influence of viscosity on the measurements. A detailed discussion of error is deferred until later, but we note here that the devices such as the pressure transducer typically have an error of the order of one percent. It would be optimistic then to take this as the error of the 
measurement since scatter in the readings and system error is larger. From the results of Chapter $V$ we have the electron density in the freestream as $1.7 \times 10^{14} / \mathrm{cc}$ and the electron temperature as $5,000^{\circ} \mathrm{K}$. Sample radial profiles of $\mathrm{n}_{e}, \mathrm{~T}_{e}$ and impact pressure are shown in Figure 5 . At this point we may discuss the choice of model test station. For the running condition chosen the model was positioned approximately in the center of the first shock diamond (4" from nozzle exit) which was found to be the most uniform area both axia11y and radially.

\subsection{Calculation of Flow Quantities}

Using the measurements just discussed and the spectroscopic results reported in Chapter $V$ we proceed to calculate the various flow quantities which wi11 enter into discussions of the results of this investigation. This analysis will be guided by the results of recent investigations by other workers using low density arc-heated facilities (see, for example, Ref. 32). A we11 known characteristic of this type of facility is that the freestream is out of equilibrium in the sense that the ions and atoms have a lower kinetic temperature than the electrons. This is due to the fact that the electrons and heavy particles are not well coupled by energy transfer between species. The high electron temperature tends to be maintained during expansion while the heavy particle temperature drops in the supersonic freestream as in normal supersonic flow.

It is reasonable to assume however, that equilibrium is approached in the arc and stagnation chamber due to the relatively high pressure $(0.5 \mathrm{~atm})$ and slow flow velocities. From the net input power of $7.7 \mathrm{KW}$ to the gas 
and the flow rate of $1.23 \mathrm{~g} / \mathrm{sec}$ we may calculate the stagnation enthalpy to be $h_{0}=2,700 \mathrm{BTU} / 1 \mathrm{bm}$. From the equilibrium calculations of Arave and Huseley (Ref. 34), and knowing the stagnation pressure we find for equilibrium argon in the stagnation chamber

$$
\begin{aligned}
& \mathrm{T}_{\mathrm{o}}=9,700^{\circ} \mathrm{K} \\
& \alpha=.025
\end{aligned}
$$

where $\alpha$ is the degree of ionization.

With knowledge of the electron temperature in the freestream we assume that the flow is chemically frozen (i.e., that recombination in the freestream is a minor effect and since the temperatures are low subsequent ionization is not important). This means that the ratio of specific heats, $Y$, is simply that of a monatomic gas, 1.67 . On this basis using the ratio of impact pressure to static pressure (= 33.5$)$ the tables of Ref. 35 show for isentropic flow

$$
\begin{aligned}
M_{\infty} & =4.75 \\
1 / \varepsilon & =\frac{P_{s}}{P_{\infty}}=3.5
\end{aligned}
$$

for which

$$
\frac{T_{\infty}}{T_{0}}=.115
$$

hence

$$
\mathrm{T}_{\infty}=1100^{\circ} \mathrm{K}
$$

where $T_{\infty}$ is the heavy particle freestream pressure.

The number density of the freestream is more difficult owing to interpretation of the $\rho_{\infty} V_{\infty}$ measurement. Instead of using this measurement 
directly, we shall estimate a set of flow-averaged quantities in the following manner, for a freestream heavy particle temperature of $1100^{\circ} \mathrm{K}$ the equilibrium data (Ref. 34) for the speed of sound gives

$$
a_{\infty}=2100 \mathrm{ft} / \mathrm{sec}=6.4 \times 10^{4} \mathrm{~cm} / \mathrm{sec}
$$

from this we conclude

$$
v_{\infty}=M_{\infty} a_{\infty}=3 \times 10^{5} \mathrm{~cm} / \mathrm{sec}
$$

Taking an appropriate jet radius from probe and spectroscopic data (see Figure 5) we may estimate the area of the jet to be

$$
A_{\text {jet }}=37 \mathrm{~cm}^{2}
$$

Therefore $\rho_{\infty} V_{\infty}=\dot{\mathrm{m}} / \mathrm{A}_{\text {jet }}=3.35 \times 10^{-2} \mathrm{gm} / \mathrm{sec} \mathrm{cm}^{2}$. Hence from our previous estimate of $v_{\infty}$

$$
\rho_{\infty}=1.1 \times 10^{-7} \mathrm{~g} / \mathrm{cc}
$$

From this an average value of the electron number density in the freestream may be calculated

$$
n_{e \infty}=\alpha \frac{P_{\infty}}{m_{h}}=4 \times 10^{13} / c c
$$

where $m_{h}$ is the mass of an argon atom. In order to make a better evaluation of $p_{\infty} V_{\infty}$ it is now possible to present another method of obtaining this quantity. From the results of Chapter IV we may introduce two more independent observations of $\rho_{\infty} V_{\infty}$. Values of the total aerodynamic drag and pressure drag coefficients with no magnetic field are given by Ref. 25 as 1.5 and 1.0 respectively. Using the measured values of these drag 
forces and the definitions of the drag coefficients

$$
C_{\mathrm{drag}}=\frac{\mathrm{drag} \text { force }}{\frac{1}{2} \rho_{\infty} \mathrm{v}_{\infty}^{2} \mathrm{R}_{\mathrm{b}}{ }^{2}}
$$

the value of $\rho_{\infty} v_{\infty}^{2}$ may readily be calculated. Then using the value of $V_{\infty}=3 \times 10^{5} \mathrm{~cm} / \mathrm{sec}$ calculated previous $1 \mathrm{y} \rho_{\infty} V_{\infty}$ is obtained. The values calculated in this manner are

$$
\begin{aligned}
\rho_{\infty} \mathrm{V}_{\infty} & \left.=2.5 \times 10^{-2} \mathrm{~g} / \mathrm{sec}^{-\mathrm{cm}^{2}} \quad \text { (from total drag }=65.6 \mathrm{~g}\right) \\
& =2.7 \times 10^{-2} \mathrm{~g} / \mathrm{sec}-\mathrm{cm}^{2} \quad \text { (from pressure drag }=48 \mathrm{~g} \text { ) }
\end{aligned}
$$

We note that these calculations are also averaged values (although the resolution is somewhat improved) since both the drag forces and $V_{\infty}$ are averaged quantities. Also obtaining radial variations is not possible by this method. The values obtained by this method lie between the probe measurements and the flow averaged mass flux. We would expect them to lie closer to the latter quantity.

We now proceed to calculate additional quantities which will be needed in later sections. In the following estimates we take $\mathrm{N}_{\mathrm{es}} \cong 5 \times 10^{14} / \mathrm{cc} ; \quad \mathrm{N}_{\mathrm{e} \infty} \cong 10^{14} / \mathrm{cc}, \mathrm{T}_{\mathrm{es}} \approx 10^{40} \mathrm{~K} \mathrm{~T}_{\mathrm{e}}=5 \times 10^{3} \mathrm{o}$. First we find the conductivity and viscosity from the equilibrium calculation of Ref. 31 .

$$
\begin{aligned}
& \sigma_{\text {shock }}=2.5 \times 10^{3} \mathrm{mho} / \mathrm{m} \\
& \uplus_{\infty}=5.4 \times 10^{-4} \mathrm{~g} / \mathrm{cm}-\mathrm{sec} \\
& \uplus_{\text {shock }}=8 \times 10^{-4} \mathrm{~g} / \mathrm{cm}-\mathrm{sec}
\end{aligned}
$$


For future reference and comparison we now calculate some microscopic quantities: The root mean square velocity

$$
\begin{aligned}
& \begin{aligned}
v_{e}=\left(\frac{3 k T_{e}}{m_{e}}\right)^{1 / 2} & =5 \times 10^{7} \mathrm{~cm} / \mathrm{sec} \text { (freestream) } \\
& =6 \times 10^{7} \mathrm{~cm} / \mathrm{sec} \text { (shock) }
\end{aligned} \\
& \begin{aligned}
v_{h}=\left(\frac{3 k T_{h}}{m_{h}}\right) & =7 \times 10^{4} \mathrm{~cm} / \mathrm{sec} \quad \text { (freestream) } \\
& =2.5 \times 10^{5} \mathrm{~cm} / \mathrm{sec} \quad \text { (shock) }
\end{aligned}
\end{aligned}
$$

where the subscript $h$ denotes the heavy particles. The mean free path for the heavy particles (Ref. 36, 37)

Neutral - Neutral

$$
\begin{aligned}
\lambda_{a a}=\frac{16}{5}\left(\frac{m_{A}}{2 \pi k T_{h}}\right)^{1 / 2} \underset{\rho}{\mu} \approx 0.13 \mathrm{~cm} \text { (freestream) } & \approx 0.03 \mathrm{~cm} \text { (shock) }
\end{aligned}
$$

Neutral - Ion

$$
\begin{aligned}
\lambda_{1 a} & =0.32 \frac{T_{h}+142}{T+11.5 T_{h}} \lambda_{a a} \\
& =0.035 \mathrm{~cm}
\end{aligned}
$$

The electron-atom mean free path is taken from Ref. 36) to be

$$
\lambda_{\mathrm{ea}}=0.2 \mathrm{~cm}
$$

The Debye length is calculated (Ref. 37)

$$
\begin{aligned}
\lambda_{D} & =\left(\frac{k T_{e}}{4 \pi n_{e}}\right)^{1 / 2} \\
& \approx 10^{-5} \mathrm{~cm}
\end{aligned}
$$


for the range of $n_{e \infty}$ and $T_{e \infty}$ estimated. The cyclotron frequency is given by Ref. 37

$$
\omega_{c}=\frac{-e B}{m_{e}}=1.75 \times 10^{11} \mathrm{~B} / \mathrm{sec}
$$

when $B$ is in Webers $/ m^{2}$. Two collision times will be needed: the collision time between electrons and ions (Ref. 37)

$$
\tau_{\text {ef }} \simeq 1.45 \times 10^{-10} \mathrm{sec}
$$

and the collision frequency between ions and neutrals

$$
v_{i a} \cong \frac{v_{h}}{\lambda_{i a}}=2 \times 10^{6} / \mathrm{sec}
$$

The ambipolar diffusion coefficient is given by (Ref. 38)

$$
D_{a \infty 0}=\frac{k\left(T_{i}+T_{e}\right)}{m_{i} \nu_{i a}} \cong 7.5 \times 10^{4} \mathrm{~cm}^{2} / \mathrm{sec}
$$

\subsection{Ca1culation of Dimensionless Parameters}

On the basis of the preceding calculations the appropriate dimensionless parameters may be evaluated.

1. The free stream Knudsen number based on the radius of the body

$$
\begin{array}{r}
\mathrm{R}_{\mathrm{b}}=0.75 \text { in. } \\
\mathrm{Kn}_{\infty}=\frac{\lambda_{\infty}}{\mathrm{R}_{\mathrm{b}}}=.1
\end{array}
$$

2. The Reynolds number at the body

$$
\operatorname{Re}=\frac{V_{s} R_{b}}{\eta_{s}} \approx 0(100)
$$


3. The Mach number and density ratio as previously calculated

$$
\begin{aligned}
& M_{\infty}=4.75 \\
& \varepsilon \approx 0.29
\end{aligned}
$$

4. The freestream ambipolar Schmidt number

$$
S c_{\infty}=\frac{\mu_{\infty}}{\rho_{\infty} D_{\text {aco }}} \approx 0.6
$$

5. The magnetic Reynolds number

$$
\operatorname{Rem}=\mu \sigma_{0} \in \mathrm{V}_{\infty} \mathrm{R}_{\mathrm{b}} \approx 0\left(10^{-2}\right)
$$

6. The interaction parameter using $\rho_{\infty} \mathrm{V}_{\infty}$ calculated from the drag coefficients

$$
s=\frac{\sigma_{o} B_{o}^{2} R_{b}}{P_{\infty} v_{\infty}}=2.0 B_{o}^{2}
$$

$\left(_{0}^{2}\right.$ the magnetic field at the stagnation point of the body expressed in kilogauss squared)

7. The Hall coefficient

$$
\mathrm{C}_{\mathrm{H}}=\frac{e \mathrm{~B}_{\mathrm{o}}}{\mathrm{m}_{\mathrm{e}}} \tau_{\mathrm{ei}} \cong 0.8 \mathrm{~B}_{\mathrm{o}} \quad \text { ( } \mathrm{B}_{\mathrm{o}} \text { in Kilogauss) }
$$

\subsection{Consistency of Data and Error}

The underlying assumption in the analysis of this type of facility is that chemical reactions are not of major importance so that the concentrations of the species are relatively constant. This will not be the case over large axial distances since the various species will tend to equibrate both energetically and with respect to concentrations. However, 
in the regions of interest, the shock layer and the freestream flow from the nozzle, the assumption of frozen flow may be justified since the scale lengths Involved are short and the flow velocities are high. We return to a discussion of the shock layer in Chapter $V$. The freestream is considered now since the assumption of no chemical reactions here lead to the value of $\gamma=1.67$, the specific heat ratio for a monatomic gas.

We expect three-body recombination to be the dominant chemical reaction in the freestream. Picking a value of $\alpha$ the rate coefficient from Ref. 39 of $1.1 \times 10^{-10} \mathrm{~cm}^{3} / \mathrm{sec}$, we have the rate equation

$$
\frac{d n}{d t}=\alpha n_{e} e^{2}
$$

which may be rewritten as

$$
\frac{\Delta \mathrm{n}_{\mathrm{e}}}{\mathrm{n}_{\mathrm{e}}}=\frac{\mathrm{n}_{\mathrm{e}} \alpha \Delta_{z}}{\mathrm{v}_{\infty}}
$$

taking $\Delta z$ as $1 \mathrm{~cm}$ we have

$$
\frac{\Delta n_{e}}{n_{e}}=10^{-2}
$$

as an estimate of the recombination effects. Since this is the principle chemical reaction we conclude that taking $\gamma=1.67$ is reasonable.

The usefulness of $\gamma$ was in the evaluation of the Mach number and $\epsilon$, which was also questioned because of the difficulty encountered in measuring the static pressure accurately. An independent and direct observation of $\varepsilon$ is available from the results of Chapter $V$ where the shock density profile is measured. The calculated value of $\epsilon=.29$ is in 
good agreement with these results.

Careful consideration must be given to the value of $P_{\infty} V_{\infty}$ which enters info the interaction parameter calculation. Four independent measurements were given, three showing consistency and the probe data being less reliable. To summarize the results were

$$
\begin{aligned}
& \rho_{\infty} \mathrm{V}_{\infty}(\text { calculated })=3.35 \times 10^{-2} \mathrm{~g} / \mathrm{sec} \mathrm{cm}^{2} \\
& \rho_{\infty} \mathrm{V}_{\infty} \text { (pressure coefficient) }=2.7 \times 10^{-2} \\
& \rho_{\infty} \mathrm{V}_{\infty}(\text { drag coefficient })=2.5 \times 10^{-2} \\
& \rho_{\infty} \mathrm{V}_{\infty}(\text { probe })=8.7 \times 10^{-3}
\end{aligned}
$$

As a final consistency check we observe that the centerline number density measured by spectroscopy $\left(1.8 \times 10^{14} / \mathrm{cc}\right)$ may be compared to that value obtained by flow averaged calculations $\left(4 \times 10^{13} / \mathrm{cc}\right)$. This agreement is reasonable allowing for gradients in the flow and the relatively low accuracy of the spectroscopic method in obtaining absolute number densities.

In evaluating the nondimensional parameters in the last section, values for the various flow quantities which seemed most accurate and consistent were selected. Obviously, other evaluations are possible. In particular, it was found most consistent to use the value of $\rho_{\infty} V_{\infty}$ calculated from the drag and pressure coefficients in the magnetic interaction parameter $S$, due to problems in interpreting the mass flux probe measurements. While an absolute error estimate for $S$ (and the other parameters) cannot be made, it is possible to examine the error in $S$ calculated in this 
manner by estimating the error in the values of $\mathrm{N}_{e} \mathrm{~T}_{e}$ used. We assume that the error in reading the magnetic field is negligible in comparison to these measurements. We take the difference between $\rho_{\infty} V_{\infty}$ calculated from the pressure and drag coefficients as an estimate of the error in $\rho_{\infty} V_{\infty}$

We will be primarily interested in the interaction parameter and the Hall coefficient in later chapters. Thus,

$$
\begin{aligned}
& \frac{\Delta T_{e}}{T_{e}}=\frac{3}{2} \frac{\Delta T e}{T}=15 \% \\
& \frac{\Delta \sigma}{\sigma} \cong \frac{3}{2} \frac{\Delta T e}{T}=15 \%
\end{aligned}
$$

where $\tau_{e}$ and $\sigma$ are calculated from the centerline variations of $n_{e}$ and $\mathbf{T}_{\mathbf{e}}$. The error in the measurement of magnetic field will be neglected. The commonly used difference method for determining the error in a calculated quantity gives

$$
\begin{aligned}
& \frac{\Delta \mathrm{C}_{\mathrm{H}}}{\mathrm{C}_{\mathrm{H}}}=\frac{\Delta \mathrm{T}_{\mathrm{ei}}}{\mathrm{T}_{\mathrm{ei}}}=15 \% \\
& \frac{\Delta \mathrm{S}}{\mathrm{S}}=\frac{\Delta \sigma}{\sigma}+\frac{\Delta \rho_{\infty} \mathrm{V}_{\infty}}{\rho_{\infty} \mathrm{V}_{\infty}}=24 \%
\end{aligned}
$$


EXPERIMENTAL OBSERVATIONS OF MAGNETOAERODYNAMIC DRAG

It was estab1ished in the first two chapters that the total magnetoaerodynamic drag was the sum of several components, each altered by the interaction of the flow with the magnetic field.

Preliminary measurements of total drag alteration such as those reported in chapter II indicated that measurement of the individual components of drag would provide better understanding of the measurements of total drag. The following approach was taken. The total drag with and without magnetic fleld and the force on the magnet were measured. Then a detailed study of the pressure distribution on the body with and without magnetic field was undertaken, leading to calculation of the pressure force. The viscous force, being the smallest component and most difficult to measure could then be calculated from these measurements assuming base drag was unaltered. Thus the total drag and individual components were available experimentally for discussion and comparison to theory.

These experimental measurements are presented in the following sections along with a description of the apparatus in each. Typical data are presented as functions of magnetic field squared, representing a dimensional form of the magnetic interaction parameter. This is done for convenience, since an evaluation in terms of an alternatively defined magnetic interaction parameter may be made by simply multiplying ${ }^{2}$ by $a$ constant factor. The data will be fitted to empirical relations and replotted in terms of the interaction parameter in Chapter vI for purposes of discussion and comparison to theory. 


\subsection{Design, Construction and Calibration of Experimental Apparatus}

The bodies and magnets used in these experiments were made as uniform as possible to eliminate systematic error. The basic body design was chosen to be $11 / 2$ " in diameter with the models containing magnets having a $11 / 2^{\prime \prime}$ afterbody and the pressure models without magnets, $1 / 2$ " after body. The magnets are fitted close to the front of the body to maximize the field as shown in Figure 6. Figure 7 is a photograph of a magnet used and a total drag model along with pressure, Lorentz drag and flat-end bodies for compairson.

The final magnets were based on experimentation with a number of jig wound magnets. The rough dimensions were as follows: 1" long by $11 / 4^{\prime \prime}$ diameter with a $3 / 8^{\prime \prime}$ core. The nose was rounded to insure a close fit at the nose, thus maximizing the field. The wire chosen for winding was \#19 Anaconda HML-coated wire, desirable for its high temperature coating. This gauge was heavy enough to take high currents and provide good heat transfer when cooling was used yet light enough to wind easily. The core material was vanadium permandur, an alloy commonly used for this purpose, heat treated according to the manufacturer's directions. The magnets once wound were fitted with cores mounted directly to the bodies at the base (see Figure 6). Typical magnet resistance was $0.5 \Omega$. Both commercial storage batteries (32) and a commercial power supply were used to power the magnets. The batteries provided practically any combination of voltage and current desired and produced a very constant power for short runs. The commercial supply was used for longer runs, testing and charging the batteries. 
The measurement of fleld strengths was performed with a Bell Gaussmeter, Mode1 120. The probe area of this instrument is approximately $1 / 8^{\prime \prime} \times 1 / 4^{\prime \prime}$ and of negligible thickness. This provides reasonable spatial resolution although not adequate for detailed measurements as a function of angle in the stagnation region.

Two tests were made. The magnetic field strengths at the stagnation point for various coil currents and the field drop-off with axial positions for a constant current were measured. Samples of field strength tests are shown in Figure 10 for several models. The normalized drop-off results are compared in Figure 11. These are compared to the theoretical dipole given by $B=B_{0}\left(\frac{R}{R_{b}}\right)^{-3}$ and also for an exponent of 3.6. The dipole field has generally been taken in theoretical treatments. It is seen that in the shock region the agreement is reasonable. To insure that positioning was not affecting the distribution severely, one magnet was moved back 0.1 " with respect to the stagnation point and these results are seen to agree with the previous results.

To measure the force on the body the following system was constructed (shown in Figure 8 ). The model was mounted on a $1 / 2^{\prime \prime}$ sting carrying power for the magnet and cooling water. This sting was suspended by two vertical leaf springs so that the force on the model is seen as a small displacement of the sting. The sensitivity can be increased or decreased by changing the thickness of the leaf spring.

This displacement was read by a Sanborn Linear Differential force transducer which also contributes to the restoring force on the sting, and provides a millivolt D.C. output linear with force. The electrical and water input to the sting were arranged so as not to interfere with 
free movement of the model. Surrounding the whole mechanical apparatus is a water cooled shield for protection of the assembly and transducer. Further shielding eliminates the drag of the sting and suspension system.

The system as a whole mounted to the overhead table assembly and the model was then positioned by a mechanical $\mathrm{jig}$ and a leve1. Once installation was completed, calibration could easlly be performed during any series of runs. This was done by positioning a small pulley behind the model and attaching a thread from the model to the pulley along the direction of displacement. The thread then runs over the pulley and desired calibration weights attached. The transducer proved to give extremely linear response. A sample calibration curve is shown in Figure 9. Typical sensitivity was $1.6 \mathrm{MV} / \mathrm{g}$, considered to be adequate for these measurements.

Another important characteristic is the time response of the mechanical system due to the short lengths of time during which the magnet could be used. Coupled with the time response of the mechanical system is the response of the electrical system. The total effect was determined by placing a weight about equal to the aerodynamic drag on the calibration system. A weight approximately the same as the magnetic field induced alteration was then suddenly added and the response measured, thus simulating the condition of an actual test. From the time the magnetic field was turned on a time of about $0.5 \mathrm{sec}$ elapsed before a reliable measurement could be made. This is due partially to the mechanical response of the system to a step input and partially to the response of the recorder (.5 sec, full scale). With an electrical 
filter added to reduce the noise the perfod elapsed before a rellable measurement could be made was increased to approximately 2 seconds.

For the Lorentz forces a similar apparatus was constructed except that the body was attached rigidly to the outer heat shield. Thus the magnet alone was suspended from the sting and the forces on the magnet were measured directly. This apparatus was not water cooled and the movable heat shield was employed in front of the body. The calibration procedure was the same as that used for the total drag model.

In order to measure pressure distributions without magnetic field solid copper bodies were used, 1 1/2" in diameter with $1 / 2^{\prime \prime}$ afterbodies. These were soldered to the impact pressure device described in Chapter II. One body was made for each angle measured and 4 taps spaced symmetrically were cut to improve response time. The same pressure transducer as in the impact pressure was used. Angles measured in this manner were $16^{\circ}, 26^{\circ}, 32^{\circ}, 48^{\circ}, 56^{\circ}, 90^{\circ}$ and the base pressure was measured at $r=3 / 8^{\prime \prime}$. In separate bodies one tap was drilled at $0^{\circ}$ and two at $8^{\circ}$. Both $1 / 16^{\prime \prime}$ and $1 / 8^{\prime \prime}$ taps were used to determine transpiration effects. These models were then suspended from the overhead table and the heat shield was used to prevent excessive heating.

The measurement of pressure distributions with field was more difficult due to the lack of space in the body. To overcome this problem in the stagnation region, the magnet core was extended through the body shell and machined to form the nose. Two $1 / 16^{\prime \prime}$ taps were then drilled through the core, one at $0^{\circ}$ and another at $8^{\circ}$.

For off-axis measurements a small copper tube was imbedded in the she11 and machined flush with the body surface. A tap was then drilled 
into the tube at the desired angle. Angles chosen were $20^{\circ}, 32^{\circ}$ and $44^{\circ}$. Only one tap was provided at each angle and several bodies were constructed in this manner. All the bodies for the magnetic field pressure experiments were attached to a $1 / 2^{\prime \prime}$ sting supported from a simple transducer housing. This was then supported from the overhead table.

\subsection{Experimenta1 Measurement of Drag and Drag Components}

A typical trace of a total drag test (unfiltered output) is shown In Figure 12. The apparent difference in the two traces is due to the mechanical crossover in the two channel recorder. The rise of the two traces is actually simultaneous. The vibration seen is attributed to mechanical noise from the roughing pump. The aerodynamic drag was measured at the beginning of a series of tests to minimize errors due to heating and these results were averaged, giving $66 \mathrm{~g}$. The total drag with field was measured in many different test series over several months using different equipment. The results of these tests and the Lorentz drag experiments are shown as a plot of percent alteration versus magnetic field squared in Figure 13.

To determine the effects of body shape on the alteration to total drag, a similar experiment was conducted with a flat ended body and magnet. These results are shown in Figure 14. The results for the hemispherically shaped body are also shown in the same figure for comparison. It is seen that the flat ended body shows a much smaller effect. 
This is not surprising since the aerodynamic drag is higher for the flat ended body. The component of drag with field is also smaller which Is attributed to the fact that the flow is both slower and less perpendicular to the magnetic field lines around the stagnation region thus reducing the Lorentz force.

The volume of the shock layer however, is greater for the flat end body. The influence of this increase as well as the influence of the conductivity of the freestream on all drag measurements is resolved in the following manner. It is shown in Chapter $V$ that the shock standoff for the hemisphere body is about $.35^{\prime \prime}$. One may readily estimate that at this position $\mathrm{B}^{2}$ has dropped about an order of magnitude. Although the conductivity does not drop much across the shock since the electron temperature is nearly constant, the velocity vector is axial in the freestream and not perpendicular with the field lines. On this basis it is reasonable to assume that neither the freestream nor a slight increase in shock standoff contribute significantly to the observed increase in drag.

The distribution of pressure on a spherical surface as given by modified Newtonian theory is

$$
C_{P}=C_{P_{0}} \cos ^{2} \theta
$$

where

$$
C_{P}=\frac{p_{0}-p}{1 / 2 \rho_{\infty} V_{\infty}^{2}}
$$

where $\theta$ is the angle measured from the centerline, and the subscript o refers to the stagnation point. Accordingly, the first step in studying pressure forces is to measure the distribution of pressure without a 
magnetic field. This will give finally, not only the pressure force on the body, but also indirect indications of the effects of flow nonuniformity.

Once the pressure has been measured as a function of angle, the total pressure on the frontal surface may be determined by integrating the pressure times the projected area upon which it acts.

The projected area of the frontal surface is given by

$$
\mathrm{dA}_{\text {pro.j }}=\cos \theta \mathrm{dA}=2 \mathrm{R}_{\mathrm{b}}^{2} \cos \theta \sin \theta \mathrm{d} \theta
$$

so that the elemental force on the frontal surface is given by

$$
d F=2 P(\theta) R_{b}^{2} \sin \theta \cos \theta d \theta
$$

From this we must subtract the force due to the base pressure to get the net force due to fluid pressure on the body. If the base pressure is taken as averaged over the area not covered by the sting, this is given by the product of the pressure and the area.

With no magnetic field the pressure distribution was measured with both $1 / 16^{\prime \prime}$ and $1 / 8^{\prime \prime}$ taps. The results are shown in Figure 15 normalized to the stagnation point value and compared to $\cos ^{2} 0$. The pressure at the shoulder and the base pressure were slightly negative which tends to confirm the static pressure value slightly below the tank pressure. The $1 / 8$ " tap gave consistently higher values than the $1 / 16^{\prime \prime}$ tap and also followed closely the $\cos ^{2} \theta$ curve.

The measurement of pressure distributions with field was conducted as follows. The pressure alteration was measured as a function of field 
and angle as shown in Figure 16 for the 1/16" tap size. This data was then averaged and added to the no-field distribution for $1 / 16^{\prime \prime}$ tap. It was then assumed that the discrepancy between the $1 / 16^{\prime \prime}$ and $1 / 8$ " holes was due to thermal transpiration and that to correct for this the following procedure was followed: we first assume that the ratio of pressure with and without magnetic field for the same tap size is independent of tap size. Hence,

$$
P(\theta, B)_{1 / 8^{\prime \prime}}=P(\theta, B)_{1 / 16^{\prime \prime}} \frac{P(\theta, 0)_{1 / 8^{\prime \prime}}}{P(\theta, 0)_{1 / 16^{\prime \prime}}}
$$

We now correct for the thermal transpiration effects on a $1 / 8$ " tap using the results of Ref. 40. Since the correction is small there is negligible error in simply assuming a constant value of $T_{h} / T_{c}$, the ratio of the gas temperature at the tap to the gas temperature at the transducer. For $T_{h} / T_{c}=4$ this amounts to a $5 \%$ correction.

These corrections were applied together to the $1 / 16$ "distributions and the results integrated giving $48 \mathrm{~g}$ the corrected distribution. Several of these distributions are shown in Figure 17 (connected to the 1/8" tap size as shown above) with the most surprising result being the pressure rise in the stagnation region. The integrated effect however, Is still a decrease in pressure force with field as shown in Figure 13.

The total drag value of $66 \mathrm{~g}$ and the pressure drag of $48 \mathrm{~g}$ with an estimated base pressure of $3 \mathrm{~g}$ permits calculation of the viscous force, found to be $21 \mathrm{~g}$ for the non-magnetic case. Assuming the base pressure does not change with magnetic field, the above procedure would allow us to calculate the change in viscous drag with magnetic field. 


\section{CHAPTER V}

\section{INVESTIGATION OF THE SHOCK LAYER}

The shock detachment distance for a blunt body in supersonic flow is a quantity frequently measured for comparison to theory. The commoniy given reason is that this quantity is conveniently measured and a theoretical treatment of the blunt body problem must predict this quantity if the entire analysis is correct. While few of the theoretical treatments reviewed in Chapter I consider drag, all determine the shock detachment distance in magnetoaerodynamic flows. In theories similar to that of Bush (Ref. 6) this quantity is predicted automatically when the body has been located during the numerical integrations.

The visually observed shock layer with and without field may be seen in the photograph of Figure 18. Although shock appears diffuse and thick, a change in shock standoff is apparent. In this investigation shock detachment distance was measured by photography and then a detailed examination of the shock layer was made by spectroscopy. The purpose of the spectroscopy was twofold: to aid in the interpretation of photographic measurements and to provide diagnostic measurements of electron density and temperature. Both of these techniques are optical, and so have the advantage of not interfering with the field while capitalizing on the self-luminous property of the flow.

\subsection{Shocks in a Partially Ionized Gas}

The formation of a shock in a partially ionized gas is complicated by the presence of three species; ions, electrons, and neutra1s, which 
may be reacting. In addition, nonequilibrium with respect to concentration or species temperature may exist and in principle, each species may pass through an individual density rise. Several works have attempted to treat these problems separately. To provide background for this chapter we will review these here. We may then determine the state of the shock encountered in this experiment by using the results of chapter III.

Talbot and Grewal (Ref. 33) have considered extensively the formation of a shock wave in a partially lonized gas where the electrons are at a different temperature than the heavy particles. Their approach is through the electron energy equation (to be discussed) and assuming no energy transfer between the electrons and heavy particles and no reactions (frozen flow). The method of solution is to assume a heavy particle shock given by a step function and numerically integrate from the freestream and the region behind the shock and match conditions at the step. The fact that the electrons are energetically uncoupled from the heavy particles results in a broad zone of elevated electron temperature upstream of the shock forced by the electron compression at the shock and transmitted upstream in the electron gas due to the high thermal conductivity of the electrons. Electron compression at the shock is found due to charge neutrality restrictions which prohibit a change in ion density (in this case heavy particle density) without an equal change in electron density. Because of the high electron temperature ahead of the shock the electron compression is essentially isothermal. These results are offered to explain the characteristic "dark space" ahead of blunt body shocks in low density arc-heated wind tunnels (see Figure 18). The reason given for the occurrence of the dark space is that the three-body 
recombination which determines the radiation from the plasma is decreased in this region due to the elevated electron temperature ahead of the shock.

These results were later confirmed in a much more general treatment of the problem by Jaffrin (Ref. 41). He treats the shock structure for varying degrees of ionization, from a very weakly ionized gas to a quasifully Ionized gas, by considering the species momentum and energy equations as well as the global conservation equations.

Chen (Ref. 42) has investigated these results in a facility similar to the one used in this work, obtaining data similar to the predicted effect, i.e., the electron temperature rises before the shock and remains relatively constant through the zone of electron compression. A constant electron temperature across the shock was also found by Sonnin (Ref. 43) conducting an experiment at low gas temperature and ionization $\left(\mathrm{T}_{\text {gas }}=200^{\circ} \mathrm{K}\right.$, $\mathrm{N}_{\mathrm{e}}=\frac{10^{1.1}}{\mathrm{cc}}$ ). Christiansen (Ref. 44) working with a cesium-seeded argon gas heater facility $\left(N_{e} \simeq 0\left(\frac{10^{12}}{c c}\right)\right)$ has made a much more detailed study Involving an investigation of thermal conductivity. Both spectroscopic studies and Langmir probes were used. His results show a small electron temperature rise across the shock, so that the temperature ratio across the shock cannot be readily predicted. In addition to the analysis of charged particle separation occurring at the shock made by Talbot and Grewal, Pipkin (Ref. 45) has considered this effect by treating a highly idealized one dimensional flow problem. His conclusion substantiates that of Ref. 33, as he finds that the electrons tend to diffuse upstream so that a charge layer is formed at the shock. The characteristic decay 
length of this layer is found to be the Debye length.

Sonnin (Ref. 43) has Investigated the question of possible separation between the charged and neutral particles by ambipolar diffusion. In order to determine the magnitude of this effect it is necessary to examine the conservation equation for both electrons and ions so as to include the effect of momentum transfer between the heavy particles. It is found that when the ambipolar scmidt number defined in Chapter III becomes small the electrons and ions pass through an independent density rise upstream of the neutral shock. This result was then confirmed by experiment.

To sumarize, the major effects which may be present at a shock in a partially lonized gas are: an elevated electron temperature ahead of the heavy particle shock due to high electron thermal conductivity, separation of the electron density rise from the heavy particle shock governed by the Debye length and charged particle - neutral particle shock separation due to ambipolar diffusion.

\subsection{The Character of the Shock Layer}

Although phenomena discussed in the preceding section may considerably complicate the phenomena in the shock region, a hydrodynamic shock in the neutral species is present and we may predict certain features before proceeding to analyze the phenomena discussed above.

Probstein and Kemp (Ref. 46) have categorized the flow through the shock by comparing the ratio of mean free path in the shock to body radius (shock Knudsen number). Thus they find that when the Reynolds number is 
1ow,

$$
\begin{aligned}
& \lambda_{\infty} / R_{b} \ll \epsilon^{1 / 2} \\
& \lambda_{\infty} / R_{b} \ll 1 \\
& \lambda_{\infty} / R_{b}<1
\end{aligned}
$$

Viscous Layer Regime

Inciplent Merged Layer Regime

Fully Merged Layer Regime

Using the results of Chapter III, we have

$$
\mathrm{Kn}_{\infty}=\lambda_{\infty} / \mathrm{R}_{\mathrm{b}} \simeq .1
$$

and

$$
\epsilon=.29
$$

hence

$$
\lambda_{\infty} / R_{b} \ll 1
$$

So we would expect merged layer flow. Further, we may estimate the shock standoff distance by the relation

$$
\begin{aligned}
& \Delta / \mathrm{R}_{\mathrm{b}} \approx \epsilon \\
& \Delta \approx 0.5 \mathrm{~cm}
\end{aligned}
$$

The effect of viscosity would be to substantially increase this value. These results correlate with the relatively large freestream knudsen number and $\operatorname{Re}=100$.

The effect of ionization or recombination in the flow is to change the density of all species. If reactions occur in the shock region where the density is changing hydrodynamically, the net result will be much more complicated. However, using a simple analysis similar to that 
used in Chapter III we may justify an assumption of frozen flow through the shock.

Calculation of the ambipolar Schmidt number in Chapter III,

$$
S c_{\infty} \approx 0.6
$$

indicates that the charged particle density rise at the shock follows the neutral hydrodynamic shock. This conclusion may be substantiated further by investigating the momentum transfer between ions and neutra1s. Collision between the heavy particles is effective in momentum transfer since the respective masses are nearly equal. Calculating the collision time for ion-atom collision:

$$
\tau_{i a}=\frac{1}{\nu_{i a}}=5 \times 10^{-7} \mathrm{sec}
$$

which we compare to the flight time through the shock

$$
\tau_{\text {chara }}=\frac{\Delta}{v_{s}}=10^{-4} \mathrm{sec}
$$

Thus,

$$
\tau_{\text {chara }} \gg T_{\text {ia }}
$$

and we conclude that many collisions between heavy particles are suffered during passage through the shock.

It remains to examine the possibility of separation of the electrons from the heavy particle shock. We calculate

$$
\lambda_{\mathrm{D}} \ll \Delta
$$

from the above results, so this effect is taken as negligible. 
It is necessary now to investigate the reactions between species. Using an analysis similar to that used in Chapter III we may justify an assumption of no chemical reactions in the shock. Thus, assuming threebody recombination as the dominant reaction, (the two-body recombination and ionization coefficients being smaller) we have again from the rate equation,

$$
\frac{\Delta \mathrm{n}_{\mathrm{e}}}{\mathrm{n}_{\mathrm{e}}}=\operatorname{con} \mathrm{e} \frac{\Delta \mathrm{z}}{\mathrm{v}_{\mathrm{s}}}
$$

taking $\alpha=10^{-12} \mathrm{~cm}^{2} / \mathrm{sec}, \mathrm{N}_{\mathrm{e}}=4 \times 10^{14} / \mathrm{cc}$ and $\Delta z=1 \mathrm{~cm}(\sim \Delta)$ we find

$$
\frac{\Delta n_{e}}{n_{e}} \approx 4 \times 10^{-2}
$$

Therefore, the assumption of chemically frozen flow is reasonable.

To summarize, we expect a viscous shock layer of substantial thickness. The density rises for the individual species are expected to occur at the same position and the flow is frozen with respect to chemical composition. From these results we conclude

$$
\frac{N_{e}}{N_{e \infty}}=\frac{N_{i}}{N_{1 \infty}}=\frac{N_{a}}{N_{2 \infty}}
$$

Bearing in mind that high electron thermal conductivity may lead to elevation of the electron temperature ahead of the shock and consequently, an unpredictable temperature increase across the shock, we proceed to discuss the optical experiments performed and returned to a more extensive analysis of the electron energy equation in the last section of this chapter. 


\subsection{Spectroscopic Methods}

The assumption of local thermodynamic equilibrium is frequently used in describing the state of a radiating gas. At low densities one may usually assume optical transparency (Ref. 47) 1.e., at some or all frequencies radiation emitted from one part of the gas passes out of the gas without being reabsorbed by another part. One then assumes that electron collisions with heavy particles and other electrons are sufficiently dominant over radiative de-excitation that the ionization and populations of atomic states are given by the familar Boltzmann-Saha equation and that the electrons have a Maxwellian velocity distribution.

With the recent increase in interest in the spectroscopy of plasmas the following more sophisticated theory has developed. The work of Bates, Kingston and McWirther (Ref. 39) and others has dealt with the state of the plasma when not all the excited states are in a Boltzmann distribution, a situation frequently occurring when the electron density is not sufficlent to maintain collistonal equilibrium at a given electron temperature. The model they assume predicts that when the electrons themselves are in a Maxwellian distribution and 3-body recombination dominates, the electron collisions tend to maintain population equilibrium of the excited states near the ionization limit at the electron temperature. Thus, in the upper states, population is determined by collisional effects and the lower states are depopulated by radiative de-excitation. The energy level at which collisional and radiative transitions become equally probable is referred to as the "thermal limit."

As a criterion for this model, Wilson (Ref. 48) has derived the 
following relation: if equilibrium is to extend to the $u^{\text {th }}$ state then the electron number density, denoted $n_{e}$, must obey the inequality

$$
n_{e} \geq \frac{6 \times 10^{13}\left(x-E_{u}\right)^{7 / 2}\left(k T_{e}\right)^{1 / 2}}{x^{1 / 2}}
$$

where $X$ is the ionization potential, $E_{u}$ the energy of the $u^{\text {th }}$ state and $\mathrm{kT}$ the electron temperature, all quantities $\mathrm{in} \mathrm{ev}$, and $\mathrm{n}_{\mathrm{e}}$ in electrons/cc. It is to be emphasized that this is a necessary but not a sufficient condition for upper state equilibrium. Setting $\mathrm{E}_{\mathrm{u}}=0$ provides an approximate criterion for the number densities and temperatures necessary for an L.T.E. model.

Values of $\mathbf{n}_{\mathbf{e}}$ are shown for two temperatures in Figure 19. One can see that the electron densities needed to maintain equilibrium in all excited states are quite high at these temperatures.

On the basis of this mode1 the most appealing spectroscopic method of measuring electron temperature appears to be the Boltzmann plot technique applied to the upper states of the atom to obtain the electron temperature. This method has been recently reviewed and applied to a similar facility in Ref. 49. The authors include much of the existing data concerning transition probabilities for argon for convenient reference. The principle of the Boltzmann plot is as follows: if a transition between state $n$ and $m$ occurs with the emission of radiation, $I_{\nu}$, at frequency $\nu$ then

$$
I_{\nu}=g_{n} A_{n m} h \nu_{n} N_{0} e^{E_{n} / k T} e
$$


where $E_{n}$ and $g_{n}$ are respectively the energy and degeneracy of state $n$; $A_{n m}$, the transition probability and $N_{0}$ the neutral species density. From Eq. (5.12) it follows that a plot of $\ln \left(\frac{I_{V}^{\lambda}}{g_{n}^{A} A_{n}}\right)$ vs $E_{u}$ on semilog paper should be a straight line of slope $\frac{1}{\mathrm{kT}_{\mathrm{e}}}$.

Measurements of electron number density may also be obtained by spectroscopy. Again the question of local thermodynanic equilibrium becomes important. Calculations based on the Boltzmann-Saha equation are ruled out. The intensity of the continuum, however, offers a measurement of number density which is at once straightforward and not strongly dependent on equilibrium.

Radiation from the continuum arises from bremstralung (free-free transitions) and recombination spectra (free-bound) and consequently depend strongly on the velocity distribution of the free electrons. Due to the small mass of the electrons and the influence of Coulomb collisions, the equilibration time for electrons is very short; therefore, a Maxwellian distribution for the electrons in the absence of external fields can nearly always be justified (Ref. 50).

A thorough review of the literature pertaining to continuum radiation may be found in Ref. 51. Briefly, the Kramers-Unsöld model of the continuum predicts that the emission coefficient is given by

$$
\epsilon_{\nu} \propto \frac{n_{e}^{2}}{\sqrt{T_{e}}}
$$

where the constant of proportionality is weakly dependent on the frequency and the electron temperature. In addition to the assumption of Maxwellian electrons the weaker restriction that all of the ions are in the ground 
state is required.

Olsen (Ref. 52) has experimentally investigated the argon continuum in a free burning arc. For the frequencles between $6000 \AA$ and $4300 \AA$ he found that the constant of proportionality was relatively frequency Independent but that the value of the emission coefficient was higher than that predicted by the theoretical model.

Calling the constant of proportionality $\mathrm{C}$ and using the relation

$$
\epsilon_{\nu}=\frac{I_{\nu}}{4 \pi d \nu}
$$

we have

$$
n_{e}=\left(T_{e}\right)^{1 / 4}\left(\frac{I_{\nu}}{4 \pi C \alpha \nu}\right)^{1 / 2}
$$

This will provide a method of determining the absolute number density although the values of $\mathrm{c}$ would be expected to be accurate to a factor of 3. The constant $\mathrm{C}$ will be taken from 01sen's data.

For purposes of studying the shock structure and the region ahead of the shock, It is convenient to normalize to the freestream value of $n_{e}$. Thus,

$$
\frac{n_{e}}{n_{e_{\infty}}}=\left(\frac{I}{I_{V_{\infty}}}\right)^{1 / 2}\left(\frac{T_{e}}{T e}\right)^{1 / 4}
$$

This method eliminates the error in determination of the constant of proportionality and has the additional advantage of being very insensitive to errors in the electron temperature ratio. 


\subsection{The Experimental Axrangement}

For purposes of optical observation the arrangement of equipment was as shown in Figure 20. Iight from the shock region vas folded by two mirrors inside the vacuum tank through a viewing port located behind the mode1, then focused at infinity by a $60^{\prime \prime}$ focal length lens. This lens is mounted on a micrometer drive platform, so that motion of the platform corresponds to changing the axial position of the focal point in front of the model. The image is then rotated through $90^{\circ}$ (for convenIence) and refocused on the entrance slit of the spectroscope, a HilgerEngis grating monochromater (Model 1000, 1 meter focal length). This Image was swept across the entrance slits by a mirror which rotates about the vertical axis to give a lateral scan. Two different lenses were used to refocus the Image; one, of $60^{\prime \prime}$ focal length gave a 1:1 image, the other of $25^{\prime \prime}$ focal length gives an image 1:0.24 (the ratio of the entrance and exit focal lengths) thus increasing the intensity. The scanning speed was varled by moving the final mirror or changing the scanning motor. Most scans were about one minute.

The intensity at the exit slits was detected by an RCA 7850 phototube mounted in a dry-ice housing to reduce the dark current and powered at $2400 \mathrm{~V}$ by a Fluke 405B power supply. The output of the phototube (voltage) was read directly across the 1 megohm input impedance of a Moseley $7100 \mathrm{~A}$ two channel strip chart recorder. The alignment of the optical system was accomplished easily by sending a laser beam backwards along the optical path starting at the exit slits and ending along the line of sight across the body nose. In this manner each of the optics could be centered. Determination of the axtal position of the body was made at 
the beginning of a run with the aid of a telescope focused on the entrance slits so that the body nose could be centered on the slits. Photographs were taken by placing the camera at the position of the first lens, so that the same interior optical path was used.

Because the system is complicated, calibration was performed in position. That is, a calibrated tungsten bulb (GE 非18A) was placed at the nose of the body. Thus, the same optical path is used for both calibration and experiments. The lateral scan speed was measured by backlighting an object of known size at the flow position and recording the output of the spectroscope. The linearity of phototube was measured by inserting filters of known optical density into the light from the calibrated 1amp. The 1ight intensity is decreased in the ratio of the $D^{\text {th }}$ power of 10 where $D$ is the optical density. Thus the 1inearity may be measured as shown in Figure 21. The micrometer s1it settings were checked by plotting the setting as a function of incident to zero intensity. The exit slits were found to be accurate within the range used. The entrance slits being set much narrower for measurements, were generally in error by $\pm 10 \mu$, scale reading, which was not considered excessive. This factor was taken into account during the absolute intensity calibration. The calibration given with the standard bulb related the brightness temperature to the lamp current. The relation between the brightness temperature and true temperature is given by De Vos and Rutgers (Ref. 53) who also supply emissivity data for tungsten. Wein's law is taken to describe the radiation; thus, 


$$
I_{c}=\epsilon(\lambda, T) T_{b u l b} \frac{2 c^{2} h}{\lambda^{5}} \exp \left[\frac{-C_{2}}{\lambda T}\right]
$$

where the value of $c_{2}$ is $1.438 \times 10^{8} \&-\mathrm{K}^{\circ}$. The spectroscope observes lateral intensity $I_{L}$ (watts $/ \mathrm{cm}^{2}$ - steradian) and the calibration source produces Intensity $I_{c} \cdot$ (watts $/ \mathrm{cm}^{2}$ - steradian). This information must be converted to the radial intensity $I_{s}$ (watts $/ \mathrm{cm}^{3}$ - steradian) through inversion and an appropriate calibration constant. The inversion is performed in dimensional increments of $n / R$ where $n$ is the number of inversion points and $R$ is the radius of the jet $(3.16 \mathrm{~cm})$. To find the calibration factor a correction is needed to account for changes in slit settings between running and calibratIon (Ref. 54). Thus

$$
I_{L}=\frac{V_{L}}{V_{c}} \frac{W_{c}}{W_{L}} \frac{W_{c}^{\prime}}{W_{L}^{\prime}} I_{c}
$$

or

$$
\mathbf{I}_{\mathbf{L}}=\mathrm{KV}_{\mathbf{L}}
$$

where

$$
\mathrm{K}=\frac{1}{\mathrm{~V}_{\mathrm{c}}} \frac{\mathrm{W}_{\mathrm{c}}}{\mathrm{W}_{\mathrm{L}}} \frac{\mathrm{W}_{c}^{\prime}}{\mathrm{W}_{\mathrm{L}}^{\prime}}
$$

where $\mathrm{V}=$ voltage read

$W, W^{\prime}=$ exit and entrance slits and subscripts $c$, L denote calibration and lateral signal respectively. The inversion scheme then returns $I_{S}$.

For the purposes of Boltzmann plots only the relative intensity is necessary. $6500 \AA$ proved to be a convenient wave length for normalization. The response of the bulb was then 


$$
\frac{I(\lambda, T)}{I(6500, T)}=\frac{\varepsilon(\lambda, T)}{\epsilon(6500, T)}\left(\frac{6500}{\lambda}\right)^{5} \exp \left[\frac{-C_{2}}{\lambda T}+\frac{C_{2}}{6500, T}\right]
$$

this quantity was then calculated for several different lamp temperatures and applied to the measurements of spectral response described in the next section.

\subsection{Spectroscopic Measurements and Results}

Using the apparatus previously described, scans of line intensity versus wave length using narrow entrance and exit slits of about $3 q_{\alpha}$. Some tracings of these are shown in Figure 22. The purpose of these scans was to determine Iine position and width as well as the presence of other spectra in the regions of interest. Identification of several Ines in a given region permitted assignment of wave length units to the trace. The appropriate slit setting is then the width of the line (in Angstroms) divided by the reciprocal dispersion ( $8 \AA / \mathrm{mm}$, first order). These results are tabulated in Table II. For the continuum measurements the area between $4300 \AA$ and $4333 \AA$ was chosen because of the relative purity, thus allowing wide exit slit settings. The cleanest region appeared to be centered at $4319 \AA$ for a slit setting of $1.5 \mathrm{~mm}$. It was a1so necessary to open the entrance slits to increase intensity. Since this has the effect of broadening the line at the exit, these measurements were repeated at several entrance settings to insure that the wings of the adjacent lines did not overlap the area of interest. Measurements of the continuum were conducted with slits of $19 q_{\mathrm{w}}$ width and a $40 q_{\mathrm{w}}$ vertical mask. The width of the jet was found from the continuum scans to be $6.32 \mathrm{~cm}$. Calibration 
tests were run at the same time with current and slit settings as follows: $15 \mathrm{amp}, 2 q_{\mu}$ entrance, $.125 \mathrm{~mm}$ exit, $V_{c}=7.5$ volts, $\mathrm{K}=2.581 \times 10^{-6}$ $\frac{\text { watts }}{\mathrm{cm}^{2}-\text { ster.volt }}$, Using the measured value of $5.0 \times 10^{-7} \frac{\text { watts }}{\mathrm{cm}^{3} \mathrm{ster}}$ at $.45^{\prime \prime}$ ahead of the body, the emission coefficient $\epsilon_{\nu}$ was calculated to be $2.7 \times 10^{-19} \frac{\text { watts }}{\mathrm{cm}^{3}-\text { ster }-\sec ^{-1}}$.

From the data of Ref. 49 we calculate

$$
C_{4319}=7.19 \times 10^{-46} \frac{\text { watts }{ }^{\circ} \mathrm{K}^{1 / 2} \mathrm{~cm}^{3}}{\text { steradian }}
$$

Using this value and a temperature of $5000^{\circ} \mathrm{K}$ in $\mathrm{Eq} .(5.15)$ gives $\mathrm{n}_{\mathrm{e}}=$ $1.8 \times 10^{14} / \mathrm{cc}$.

For line measurements it was desirable to pick lines which were sufficiently isolated to permit opening the slits slightly wider than necessary. Thus a small error in wave length setting would not delete the wing of the line from the measurements.

Figure 23 shows a scan of the 4300 line and the underlying continuum. The continuum intensity amounts to about $5 \%$ of the centerline value of the line which was one of the widest measured. Since this error is smaller than the reproducibility of a trace, it was decided to neglect this correction. In order to retain spatial accuracy and eliminate the need for separate calibration of each run, the magnetic field was pulsed at approximately one second intervals. Thus a lateral intensity distribution with and without magnetic field was obtained, as shown in the tracing, Figure 24. This method has the additional advantage of keeping the 
magnet cooler and the field more uniform.

In order to recover the radial profiles from the lateral measurements, the recorder output was first folded and averaged. The method used was generally to try to obtain the best agreement about the intensity maximum region. Data was rejected if the disagreement in the wings seemed excessive. The well-known Abel integral equation was then applied and inverted to recover the radial values by a numerical approximation due to Pearce (see Ref. 55). Figure 25 shows a sample inversion of some of the continuum data; the same technique was applied to the line data.

For the purpose of making Boltzmann plots, lines were selected which has sufficient intensity and isolation and in addition the availability of data concerning transition probabilities was taken into account. To insure that equilibrium with the freestream could be expected for these lines, Figure 19 may be used by placing the upper level energy of the line on the graph (as shown for the $4300 \AA$ line, the lowest lying line selected). The following lines were measured: $4300 \AA, 4333 \AA, 4345 \AA, 4510 \AA$, $5606 \AA$, and $5650 \AA$. The entrance slits were held to $3 q_{4}$ with both $10 q_{4}$ and $40 q_{\downarrow}$ vertical masks.

The necessary data for each line used along with the spectral sensitivity of the system as measured with the standard lamp is shown in Table II.

The degree of scatter in the final data made construction of a straight line difficult, so that a more sophisticated technique was required. Accordingly, several sets of data were taken and each set was plotted on semi-iog paper. Then the data could be superimposed with a 
relative shift in the direction of the logarithm axis. This corresponds to multiplication by an arbitrary constant. Thus, the data were easily averaged making construction of a straight line much more accurate. A sample Boltzmann plot at $0.15^{\prime \prime}$ is shown in Figure 26 . The centerline temperatures obtained from this method are presented in Figure 27. Similarly, ta for the radial variation of freestream temperature was shown in re 5 .

Us ise temperature measurements the electron density measurements may be corrected for the rise in temperature. These results are also shown in Figure 26, normalized to the freestream value taken at 0.35". Neglecting the temperature correction Figure 27 shows the variation in the shock for several field strengths. While the absolute values are not considered highly accurate, as a relative measurement these results are considered quite good. The error in this technique is of the same magnitude as the scatter in $\mathrm{n}_{\mathrm{e}}$ near the centerline $(\sim 10 \%)$.

To obtain the absolute values of the electron number density the constant of Eq. (5.15) was evaluated. Then with the absolute intensity of the continuum known from previous calculations, the electron number was obtained. The spectroscopic measurements show expected results in that the number density and electron temperature profiles are shifted upstream when the field is applied. An unexpected result is that the number density increases at the stagnation point. This effect is confined to the stagnation region as shown in Figure 28. 


\subsection{Photographic Measurement of Shock Standoff Alteration with Magnetic Field}

Photographic techniques were used by Zeimer (Ref. 10) and Seeman (Ref. 31) In measuring magnetoaerodynamic shock standoff distances. Balley and SIms (Ref. 56) have presented photographic measurements of shock standoff under conditions similar to the flows of this investigation at low Reynolds number. Utilizing the self-luminosity of the flow a photograph is taken and then scanned with a photodensitometer. They assume that the upstream edge of the shock is defined by this point where the intensity starts to rise. This is assumed to be the rise in species number density.

A modification of this technique was used to measure the alteration to shock standoff. In the region of intensity rise, photographs (such as Figure 18) with and without magnetic field show the same initial profile (shown in Figure 29). Thus we superpose the two intensity traces with and without magnetic field and then move one profile relative to the other until they coincide. The distance moved is assumed to be the alteration to shock stand-off. This method is illustrated in Figure 29.

We are assuming a simple model for the flow in this region, that the Initial density and temperature rise are being shifted axia11y but do not change shape. This model is reasonable in light of the discussion of the preceding section and the spectroscopic data. 
Using this method photographs were taken at various magnetic fields at one flow condition. The exposures were made at $F / 22$ and $1 / 10 \mathrm{sec}$. through a ID neutral density filter. The film was developed for 8 minutes in Kodak D76 developer. The photodensitometer used was a JoyceLoeble Scanning Microdensitometer. The total system magnification accounting for the camera and densitometer was 5.6. The purpose of the long time exposure with $1 \mathrm{D}$ filter was to eliminate some of the intensity variations due to plasma noise.

The sharp intensity rise on the body nose (due to reflection) provided a convenient point to measure the alteration. As another measurement of shock standoff without field, a scratch was placed on the film at the nose of the body. The method of Ref. 56 was then followed to obtain absolute shock standoff distance. Reasonable agreement was obtalned. Figure 30 shows the results of this experiment and the alteration as measured spectroscopically.

\subsection{Observations in the Freestream}

In this section some aspects of the formation of the bow shock wave and the partially ionized flow ahead of the shock are considered in more detail. In particular, two effects were noticed in the freestream during the course of this investigation, a change in the color of the flow (to red) when the magnetic field was applied and the "dark space" immediately upstream of the shock (see Figure 18).

The typical plume of argon plasma produced in this type of facility has been discussed by Brewer and McGregor (Ref. 57). The length of these 
plumes formed as the flow exhausts through the nozzel and into the large vacuum chamber is quite long, often several feet, and pinkish-red in color. The luminosity of plume is attributed to energy storage in the argon metastable excited levels. Since downward transitions are improbable for these levels, atoms excited to these metastable levels tend to be convected downstream. However, at any later time electron collisions can excite these atoms to higher states from which transitions downward to the ground state are possible, thus the energy stored in the metastable states is continually lost along the flow. The metastable states of argon are at $11.5 \mathrm{ev}$ and 11.7 . The states above these can generate $a$ variety of lines through downward transitions, but many of the red lines lie within a few ev of the metastable states. The electron temperature $\left(\sim 5 \times 10^{-3} o_{\mathrm{K}}\right)$ indicates that many electrons available for collisions are In this range so that upwards collision to states which have red lines is probable, for example, argon $6965 \AA$. In addition, 1 ines such as $6965 \AA$ have strong transition probabilities so that the argon spectrum in the plume tends to be red in color. However, the color change due to magnetic field was unexpected and will now be discussed.

Figure 31 shows several lines of argon in a modified Boltzmann plot at $0.65^{\prime \prime}$ ahead of the body. Instead of plotting $I \lambda / g A$ as the ordinate, $\mathrm{Im} / \mathrm{I}$, the ratio of 1 ine Intensity with and without field is plotted, giving $\frac{1}{k} \frac{T_{m}-T}{T_{m} T}$ as the slope. Thus, Figure 31 shows an electron temperature decrease at this position when the field is applied. The reason for the red color change is seen to be a 3-fold increase in the intensity at $6965 \AA$, a relatively strong line of argon in the red. 
To explain the drop in temperature we examine the electron energy equation. In one dimensional steady flow following Ref. 41, we have

$\frac{3}{2} n_{e} V k \frac{d T_{e}}{d z}+\frac{n_{e} V k}{V} T_{e} \frac{d V}{d z}-\frac{d}{d z}\left(K_{e} \frac{d T_{e}}{d z}\right)-\mu_{e}\left(\frac{d V}{d z}\right)^{2}=Q_{e 1}+Q_{e a}+q$

where

$$
\begin{aligned}
& \mathrm{K}_{e}=\text { electron thermal conductivity } \\
& \mathrm{H}_{\mathrm{e}}=\text { electron viscosity } \\
& \mathrm{Q}_{\mathrm{el}}=\text { rate of energy transfer by electron ion collisions } \\
& Q_{\text {ea }}=\text { rate of energy transfer by electron atom collisions } \\
& \mathrm{q}=\text { rate of energy transfer by } 3 \text {-body recombination }
\end{aligned}
$$

For the thermal conductivity and viscosity the values for a fully Ionized gas will be used since the degree of ionization is sufficiently high so that coulomb collisions dominate. Thus for the thermal conductivity (Ref. 37)

$$
\mathrm{K}_{\mathrm{e}}=\frac{4.67}{\ln \Lambda} \times 10^{-12} \mathrm{~T}^{5 / 2}
$$

and the viscosity is given by Ref. 58

$$
\mu_{e}=R_{e} \frac{4 m}{15 k}
$$

Considering the right side of Eq. (5.22), the rate of electron-ion energy transfer has been given by Byron and Petschek (Ref. 59) as 


$$
Q_{e I}=\frac{n_{e}^{2} e^{4}}{m_{a}}\left(\frac{8 \pi m}{k T_{e}}\right)^{1 / 2}\left(\frac{T_{h}}{T_{e}}-1\right) \ln \left[\frac{9\left(k T_{e}\right)^{3}}{8 \pi m_{e} e^{6}}\right]
$$

at $\mathrm{T}_{\mathrm{e}}=5000^{\circ} \mathrm{K}, \mathrm{N}_{\mathrm{e}}=10^{14} / \mathrm{cc:} \mathrm{Q}_{\mathrm{ei}}=1.3 \times 10^{7} \mathrm{erg} / \mathrm{sec} . \mathrm{cm} .^{2}$. For purposes of calculation, however, we take a simpler approximation (Ref. 37) that the rate of temperature change is proportional to the species temperature difference and the collision rate

$$
\frac{d T}{d t}=\frac{T_{i}-T_{e}}{T_{e i}}
$$

Thus,

$$
Q_{e 1}=3 / 2 n_{e} k\left(\frac{T_{i}-T_{e}}{\tau_{e i}}\right)
$$

We expect that the rate of electron atom energy transfer would be s.aller than the electron ion rate since the respective cross sections differ greatly in magnitude while the densities do not. To see this, the ratio of energy transfer rates may be expressed approximately as (Ref. 41):

$$
\frac{Q_{e a}}{Q_{e 1}}=\frac{n_{a}}{n_{1}} \frac{\sigma_{e a}}{\sigma_{e 1}}
$$

where $\sigma_{e q}$, the atom electron cross section is cypically $10^{-17}-10^{-16} \mathrm{~cm}^{2}$ and $\sigma_{e 1}$, the ion electron cross section is typically $10^{-13}-10^{-12} \mathrm{~cm}^{2}$ (Ref. 25). Therefore, we expect $Q_{e a} \ll Q_{e i}$ and that we may neglect $Q_{e a}$ in Eq. (5.22). The three body reaction rate is given by Ref. 44 and Eq. (3.32) as

$$
q=\epsilon \frac{d n_{e}}{d t}=\alpha n_{e}^{2} \epsilon
$$

where $\varepsilon$ is the energy released during the reaction. The three-body recombination process tends to compete with equilibration since it adds 
energy to the electron gas. This energy is released to the third body, a free electron during the recombination reaction. Evaluation of the reaction rate 18 made difficult by the possibility that some absorption may exist in resonance transitions. This problem has been considered by Chen (Ref. 60) who finds that for argon gas at these conditions, $\epsilon \simeq 1 \mathrm{ev}$, provided the plasma is optically thin, but 6 can possibly be an order of magnitude higher with trapped resonance radiation. We shall assume that the plasma is completely optically thin and bear in mind that this value of $e$ may be conservative. The value of $q$ is then

$$
q=1.7 \times 10^{6} \mathrm{erg} / \mathrm{sec} . \mathrm{cm}^{2}
$$

We may further neglect the flow work and viscous terms (containing $\frac{\mathrm{dV}}{\mathrm{dz}}$ ) in the region of interest since $\mathrm{V}$ is very nearly constant and in the negative $z$ direction. Eq. (5.22) then becomes under the above assumptions:

$$
-\frac{3}{2} n v \frac{k d T e}{d z}-k_{e} \frac{d^{2} T_{e}}{d z^{2}}+\frac{3}{2} \frac{n k T e}{T_{e i}}=\frac{3}{2} \frac{n k T_{1}}{T_{e i}}+q
$$

where $T_{1}, q$ and $K_{e}$ will be taken as constant. This equation has an exponential homogeneous solution of the form $T=A e^{-\beta z}$ where

$$
\beta=\frac{3}{4} \frac{n_{e} k}{k_{e}} v_{\infty} \pm \frac{1}{2} \sqrt{\frac{9}{4}\left(\frac{n_{e} k v_{\infty}}{k_{e}}\right)^{2}+6 \frac{n_{e} k}{k_{e} \tau_{e 1}}}
$$

When equilibration is neglected entirely the characteristic length for an e-fold decrease in electron temperature

$$
\frac{1}{\beta}=\frac{\mathrm{K}_{\mathrm{e}}}{3 / 2 \mathrm{n}_{\mathrm{e}} \mathrm{V}_{\mathrm{k}}^{\mathrm{k}}} \cong 10 \mathrm{~cm}
$$

This is the type of situation considered in (Ref. 33 and 44). Thus, a relatively extensive region ahead of the shock would show an elevated electron temperature. 
If equilibration is accounted for, $\beta \cong 2 \mathrm{~cm}$. from the previously estimated values. This value would be somewhat larger if $q$ were taken into account. These two values of $\beta \cong 2 \mathrm{~cm}$ with equilibration and $10 \mathrm{~cm}$ without equilibration represent limiting scale length values for the extent of the zone of elevated electron temperature. Since equilibration is important as shown previously, the calculations tend to explain the fact that such a zone was not observed experimentally (Cf Figure 27).

We now consider the interaction of the magnetic field with the conducting freestream flow. This may be estimated by inserting the Lorentz force in a momentum balance. Thus

$$
\rho_{\infty} v_{\infty} \frac{d v_{\infty}}{d z}=-\sigma v_{\infty} B^{2}
$$

For a field of 200 gauss and $\sigma_{\infty}=1.5 \times 10^{3}$ mho/meter and $\Delta z=1 \mathrm{~cm}$, we have

$$
\frac{\Delta V_{\infty}}{V_{\infty}}=\frac{-\sigma B^{2} \Delta z}{P_{\infty} V_{\infty}}=-0.8
$$

We conclude from this order of magnitude estimate that the flow is slowed very slightly by the magnetic field thus forcing the observed temperature drop by enhanced electron-ion equilibration.

Although the flow velocity changes only slightly the zquilibration rate is sufficient to cause a change in the relative line populations. This Idea is further substantiated by Figure 32 which shows that the $6965 \AA$ line of argon (which shows the strongest intensity change with magnetic field) is very strongly altered even at the outer fringes of the jet. This would be expected if one considers that at the outer regions the 
magnetic field lines are more nearly normal to the flow, thus the flow would be even more slowed here than at the centerline.

The cause of the "dark space" In the luminosity just ahead of the shock observed here and in other investigations then remains to be explained. Grewal and Talbot (Ref. 33) attribute this to the zone of elevated temperature causing a decrease in recombination, hence in line radiation from the plasma.

If we assume as they do that the 3-body recombination coefficient is given by

$$
\alpha=5.6 \times 10^{-27}(\mathrm{Te})^{-9 / 2} \mathrm{n}_{\mathrm{e}}
$$

then the intensity ratio of freestream to dark space is given by

$$
\log _{10} \frac{I}{I_{\infty}}=\log _{10} \frac{\alpha}{\alpha_{\infty}}=\frac{9}{2} \log _{10} \frac{T_{e \infty}}{\mathrm{Te}}
$$

The value of $\log I / I_{\infty}$ may be found from Figure 29, since

$$
\log _{10} \frac{I}{I_{\infty}}=D-D_{\infty}
$$

the film density change. Thus we find

$$
\frac{T}{T_{\infty}}=1.1
$$

so that an extremely sma1l rise (10\%) in electron temperature while the number density remains constant can account for the "dark space" in the manner offered by Ref. 33. This type of accuracy is beyond the accuracy of Boltzmann plot technique we have used, so that the explanation can neither be confirmed or discounted. However, the fact that such a small difference 
which are then solved for $k_{j}$. This method is compatible with the concept of standard deviation of the data which is then defined (Ref. 61)

$$
\delta=\sqrt{\frac{\left(f\left(x_{1}\right)-f_{1}\right)^{2}}{n-2}}
$$

where $n$ is the number of discrete data points.

By inspection of the data and because no directly applicable expressions exist, the data for the force on the magnet and the pressure alteration are fitted with linear relationships. Since considered as a percentage alteration these quantities must pass through the origin, the following function is taken.

$$
\mathbf{f}=\mathbf{a B} \mathbf{B}^{2}
$$

Applying the above process results in

$$
\begin{aligned}
& \frac{\text { Lorentz drag force on magnet }}{\text { Total no field drag on body }} \times 100 \%=1.88 \mathrm{~B}^{2} \% \\
& \frac{\text { Alteration to Pressure force }}{\text { Total no field drag on body }} \times 100 \%=-.49 \mathrm{~B}^{2} \%
\end{aligned}
$$

Inspection of the data (Figure 13) also shows that it is not reasonable to apply a linear function to the total drag alteration. Since the Hall effect (which depends on $B$ rather than $B^{2}$ ) is expected to be import ant a series of the form

$$
E=a_{1} B+a_{2} B^{2}+a_{3} B^{3}+\ldots
$$

could be considered. A two term expansion of this form however, proved to give a bad fit, although the general features were reproduced. While higher order expressions chould be taken, physical interpretations of empirical expressions derived in this manner are difficult to make. A 
simple function of the form

$$
f=\frac{a B^{2}}{1+a_{1} B^{2}}
$$

may be fitted to the data. The motivation for picking this particular form will be discussed later in this section. The following procedure was used to fit the function. The parameter $a_{1}$ may be determined graphically by trial and error since the function is less sensitive to $a_{1}$ than to a; $f$ is then linear in $\mathrm{X}$ where

$$
X=\frac{B^{2}}{1+a_{1} B^{2}}
$$

The constant a may readily be evaluated as before. The function $f$ determined is

$$
\frac{\text { alteration to total drag }}{\text { total no field drag on body }} \times 100 \%=\frac{1.55 \mathrm{~B}^{2}}{1+.01 \mathrm{~B}} 2 \%
$$

The data for the alteration to the shock standoff as shown in Figure 30 was also fitted to a linear relationship. Thus,

$$
\frac{\text { alteration to shock standoff }}{\text { body radius }}=0.96 \times 10^{-2} \mathrm{~B}^{2} \%
$$

The relationships for the drag and shock standoff alteration may be expressed in terms of the interaction parameter given by Eq. (3.30). The resulting expressions along with the standard deviation $\delta$ about the curve fit are summarized in Table 3.

These expressions are shown plotted with the data in Figure 33 . In addition to these functions, we may deduce a function for the viscous drag alteration by subtracting the measured components from the total drag alterations to the measured components. 
Thus,

$$
\begin{aligned}
& \frac{\text { a1teration to viscous force }}{\text { total no field drag }} \times 100 \%= \\
& {\left[\frac{1.55}{1+.01 \mathrm{~B}^{2}}-1.38\right] \mathrm{B}^{2} \%}
\end{aligned}
$$

This would be reasonable for the range of $B^{2}=10$ to 20 , where the function is negative.

The function (Eq. 6.9) selected to represent the total drag was somewhat unusual and should be discussed further with regard to its behavior. Judging from the calculated standard deviation this function provides a good fit, which is sufficient to justify using it to represent the data. However, this function is also attractive since some theoretical arguments have been put forth to indicate that beyond a certain value (shock liftoff) the value of the drag can no longer increase due to the fact that the magnetic force is balancing the force of the flow through the shock (Refs. 3 and 25)。While it is generally recognized that this idea is largely hypothetical based on elementary considerations and does not account for such effects as change in shock shape, there is some validity to the argument especially in the laboratory where the flow is not unbounded and there is a maximum value for the flow momentum. We would expect then that the increase in drag would taper off at high interaction and possibly approach a constant value. The function chosen for the total drag data has this behavior since it tends to a constant value as $B^{2}$ becomes large. This value of the function represents the point at which shock layer liftoff could conceivably occur and no further increase in drag may be possible. The ultimate value of the function is $155 \%$. We may estimate the inceraction necessary to produce this type of drag alteration and the 
approach to shock 11ft-off. For example, at $S=200$, the drag alteration would be one-half its final value and at $S=400$, two-thirds.

\subsection{Comparison of Magnetoaerodynamic Drag Data to Theory}

The results in Ref. 25 are presented as magnetoaerodynamic drag coefficients which are functions of the interaction parameter $S$. These values may easily be converted to a percent alteration using the value of the coefficient at $S=0$. The empirical curve (Eqs. 6.9 and 3.30) for total drag is shown in Figure 34 with the following results from the theory of Ref. 25: inviscid, no Hall effect; inviscid, with Hall effect; viscous, no Hall effect; and a composite theory accounting for both the Hall and viscous effects at high interaction. In order to correctly refer the data to these theories it was necessary to account for the difference between $\epsilon=.29$ for experiment and $\epsilon=.20$ for the theories. This was done by correlating the data and theory by the nondimensional parameter Se as explained in Chapter $I$. Thus, the different theories could be readily converted to $\varepsilon=.29$. In the Hall effect theories the Hall coefficient varies with $B$ in the same manner as experiment.

The composite theory of Ref. 25 is based on the theoretical result that the viscous solution merges with the inviscid solution for moderate interaction. It is supposed that this also occurs when the Ha11 effect is present, so that for moderate interaction the inviscid, Hall effect drag coefficient is taken to be the solution. To construct the percent alteration then the following expression is taken: 


$$
\% \text { alteration }=\frac{C_{D}\left(S, C_{H}, R_{e}=\infty\right)-C_{D}\left(0,0, R_{e}\right)}{C_{D}\left(0,0, R_{e}\right)} \times 100 \%
$$

Since $C_{D}\left(0,0, R_{e}\right)$ is larger than $C_{D}\left(0,0, R_{e}=\infty\right)$ the theory will show a negative alteration if $S$ is taken too small. Thus, the composite theory does not pass through the origin in Figure 34 .

Inspection of the viscous and composite theories in Figure 34 shows that the onset of substantial increases in total drag is considerably delayed by viscosity. This theoretical result has been attributed to a near cancelation of effects (Ref. 25). The pressure and viscous drag coefficients both increase as the Reynolds number decreases in purely aerodynamic flow. At a particular Reynolds number the effect of magnetic interaction is to cause an increase in the Lorentz force which is nearly cancelled by a decrease in pressure and viscous drag. Presumably the viscous coefficient goes to a constant value at some value of $S$ and so can cause no further effect while the force on the magnet keeps increasing. This explains qualitatively the delayed feature of the viscous theory curve.

While experimental conditions indicate that the flow is very viscous, the experimental drag data does not repxoduce the qualitative feature of the viscous theory curve of Porter (Ref. 25). The best qualitative agreement is found with the inviscid theories, and at high interaction parameter with the composite theory. For the present we will not be concerned with quantitative agreement and try to understand more completely the qualitative features of the data.

In the experiment it was found that the viscous drag on the body was 
changed bery little when the magnetic field was applied. Eq. (6.11) would show that at $25 \mathrm{Kg}^{2}$ the alteration to the total drag as a result of alteration to the viscous drag is about 4 percent. This can be explained by the fact that the axial component of the viscous force near the stagnation region is small as compared to the total axial viscous force for an hemispherical body with an afterbody. This is because the axial component of the shear stress at the stagnation region is small and the stagnation area is likewise small. Since the electromagnetic effect is concentrated only near the stagnation region, it is perhaps not surprising to see that the change in viscous drag due to magnetic field is small as compared to total viscous drag.

According to the experimental evaluation of the interaction parameter, a 4 percent change in total drag due to viscous effect vould correspond to an interaction parameter of 50. Viscous theory hovever, predicts that the viscous drag vould have approached a constant value at this interaction parameter since this is the range where the inviscid and viscous drag coefficient are nearly identical and the viscous theory predicts a substantial increase in drag with interaction. This is also the range where the composite theory may begin to be applicable.

Part of the reason for the discrepancy between the viscous theory and experiment is due to the small angle assumption of the theory. In the region near the stagnation point, the assumption of $\sin \theta \approx \theta$ is justifiable. The extension of the calculation to $45^{\circ}$ using the above assumption would tend to overestimate the change in viscous drag due to magnetic field. Another factor that influences the theoretical result is that Porter only calculated viscous drag up to $45^{\circ}$, and neglected the viscous drag at large angles and of the afterbody. This would tend to 
reduce the total drag coefficient thus dramatize the decrease in viscous drag coefficient.

St111 another factor may contribute to the discrepancy and that is the assumption of a thin shock in the theory. At very low Reynolds numbers encountered in the experiment the shock is actually quite thick. Thus, the actual flow profile around the body may be quite different from a theoretical profile calculated assuming a thin shock.

These reasons indicate why the experiment does not have the qualitative feature of the viscous theory curve in Figure 34. The viscous theory over-estimates the amount that the viscous component of drag is altered when the magnetic field is applied. While the velocity profile around the body is certainly different than the inviscid theory would predict, the principle alteration of the drag on the body is the Lorentz force and the alteration in pressure force. The viscous force stays relatively constant because it is important mostly outside the region of interaction around the nose. The reason that the inviscid theory and the data do not agree quantitatively is that the theory which gives the alteration to drag as a percentage is based on the inviscid drag coefficient at no magnetic field whereas the experiment is based on a viscous drag coefficient. Other reasons are that the velocity profiles differ and the experimental determination of $S$ may be off. Suitable modifications to the theory to account for quantitative discrepancies will be discussed later. We now discuss comparison of the data with theories which include the Hall effect. We have noted previously that the total drag data curve agrees qualitatively with the inviscid Hall effect theory and also with the composite theory at. high interaction. However, to fully understand 
whether or not this behavior is completely in agreement with the theory It is necessary to examine individually the Lorentz and pressure forces.

We consider first the Lorentz force. In Porter's theory (Ref. 25) this force is found to be independent of viscosity. Apparently this result is again a cancelation of effects. The velocity for the more viscous cases is compensated by the increase in the interaction volume due to the increase in shock standoff with viscosity. Because of this theoretical result it is easy to formulate the percent alteration to the total drag for the Lorentz force component including both the Hall and the viscous effects. This is done in a manner analogous to the composite theory for the total drag alteration. Thus,

$$
\% \text { alteration, Lorentz component }=\frac{C_{D L}\left(S, C_{H}, R_{e}=\infty\right)}{C_{D}\left(S=0, C_{H}=0, R_{e}\right)} \times 100 \%
$$

Figure 35 shows the empirical relation for the alteration of the total drag force due to the force on the magnet plotted with the theory for $\mathrm{C}_{\mathrm{H}}=0$ and $\mathrm{C}_{\mathrm{H}}=.56 \sqrt{\mathrm{s}}$, the value of $\mathrm{C}_{\mathrm{H}}$ determined experimentally. The reaction force on the magnet is found to increase more rapidiy with $S$ than would be predicted by the Hall effect theory.

The pressure component of total drag may be examined in a similar manner. Figure 36 shows the experimental curve for the alteration in total drag due to change in pressure drag and three theoretical curves, a viscous and inviscid theory (no Hall effect) and an inviscid Ha11 effect theory. The manner in which the theoretical curves are generated was explained in Chapter II.

Figure 36 shows a surprising qualitative difference. The inviscid. 
Hall effect theory shows that the pressure force on the body increases with interaction whereas the non-Hall effect theories show a decrease. The experimental data shows that the pressure force decreases.

In addition to the pressure force on the body, we may also examine the experimental pressure distributions. (CF. Figure 17). These show that the pressure is locally relieved at angular positions where the projected area is large $\left(\sim 20^{\circ}\right.$ to $\left.40^{\circ}\right)$. The stagnation pressure rise contributes only a slight increase in pressure force since the projected area over which this pressure acts is small.

We cannot explain why the inviscid Hall effect theory of Ref. 25 shows that the pressure force on the body is increased. However, it may be noted that this does not seem to be compatible with the notion that the shock layer may eventually lift completely off the body and totally relieve the pressure.

In addition to the disagreement between theory and experiment, with regard to the pressure force, the observed pressure rise at the stagnation point would not be expected from theoretical results since it is assumed that there is no interaction along the central streamline. A plausible explanation of this phenomenon is that electrons tend to follow the magnetic field lines. Thus, for convergent field lines, as in the present case of a dipole magnet at the center of a hemisphere, the electrons are being channeled toweard the stagnation region. At the same time the electrons also follow the fluid motion along the streamline since we have charge neutrality and no ion slip. In a weak magnetic field with $\mathrm{C}_{\mathrm{H}}<1$, the collisions between electrons and ions and neutrals are strong enough so that the electrons move along the streamlines. In a 
strong magnetic fleld with $\mathrm{C}_{H}>1$, as in the present case, the drifting of the electrons along the field lines becomes importnat, Because of charge neutrality and no ion slip, the ions and neutrals also tend to be pulled toward the stagnation region. The net result is an increase in density in the stagnation region resulting in an increase in temperature and pressure in the stagnation region.

From this and the previous discussions we conclude that while some agreement seems evident between the various theories and experimental data for total drag, closer inspection of the various components of the drag has shown that this agreement is not good qualitatively.

The theoretical and experimental results for shock standoff alteration are compared in Figure 37. Here the inviscid and viscous theories lie close together and are qualitatively similar to the data. The experimentally measured alteration to shock standoff seems to be less than that of either the viscous or inviscid theories.

Another reason for this difference is undoubtedly the influence of the Hall effect which is accounted for in both the inviscid and composite theories. However, we did not observe the inflection in the data which both these theories predict. Again no reason has been given for these inflections. Possibly the scatter in the data was too large and magnetic field too low to permit this observation. Because the shock structure in experiment and theory differs substantially it is difficult to make a meaningful quantitative comparison. It would seem that the non-Hall effect theories predict too high an alteration while the Hall effect theories predict too low an alteration. No arguments against the viscous theory similar to those made in the discussion of the drag can be made 
here. However, the Hall effect theory which is already under question concerning the drag should also be questioned here because of the unexplained inflection in the alteration. 


\section{CHAPTER VII \\ SUMMARY AND CONCLUSIONS}

In the course of this investigation, we have observed the phenomena of magnetoaerodynamic flow for a simple blunt body problem. Observation of the expected increases in magnetoaerodynamic drag and shock standoff has been combined with supplementary investigation of the non-equilibrium properties of the ionized gas flow.

Spectroscopic measurements in the shock layer and freestream have been made. The fact that the flow around the body is very viscous was confirmed by experimental measurements of the shock profile. This profile was found to be similar to theoretical models for merged layer flow (Cf. Ref. 62). In the freestream and in the shock the flow was nonequilibrium. While the drag is not affected by the non-equilibrium freestream, the small amount of interaction is seen to account for the observed color change when the magnetic field is applied. It should be emphasized that this color change was observed in argon and depends on the metastable states of argon. Thus, for a different gas other color changes or no color change at all may be observed. Behind the shock the non-equilibrium effects tend to keep the electron temperature constant. Thus, the assumption of constant conductivity seems quite reasonable for this experiment.

The experimental drag measurements show an substantial increase in total drag at a magnetic field of several thousand gauss. Further measurements show that the increase is due to the increase in Lorentz drag, with the pressure drag actually decreasing and the viscous drag decreasIng slightly. The shock standoff shows a substantial increase with magnetic field. 
The pressure and spectroscopic measurement furthermore show that pressure, density and temperature of the gas increase with magnetic field near the stagnation region. This is analogous to "mirror effect" and indicates that Hall effect is important in this experiment.

An attempt is made to compare the experimental result with different calculations especially that of Porter's (Ref. 25). Although both the calculation and the data agree in overa11 trend of increase in drag and shock standoff with magnetic field, they show some disagreement in details. They are:

1. Experiment shows an increase in density and pressure near the stagnation region. The constant density model by its assumption would exclude such changes.

2. Experiment shows a decrease in pressure drag with magnetic field and theoretical calculation with Hall effect actually shows an increase with magnetic field. It is not known at present why the theoretical calculation shows an increase of pressure force with Hall effect and yet a decrease when Hall effect is neglected.

3. In the shock standoff measurement, again the data do not agree qualitatively with the Hall effect theory. No physical explanation has been given for the existence of the inflection point on the theoretical curve.

In conclusion the present experimental investigation shows that substantial increase in drag with high magnetic field is possible. The experiment further shows that prediction based on present theories should be treated with care. For high magnetic field with Hall effect, constant 
property model breaks down. As yet there is no completely satisfactory Hall effect theory. For even higher magnetic fleld to achieve 1ift-off of the shock layer, depending on the degree of lontzation, ion slip can play an important role. Thus any complete theory should take this into account at high magnetic field as in Hall effect in moderate magnetic field. 
1. Kantrowitz, A. R., "A Survey of Physical Phenomena Occurring in F1ight at Extreme Speeds," Proceedings of the Conference on High Speed Aeronauties, p. 335, Edited by A Ferri, N. J. Hoff and P. A. Libby, Polytechnic Institute of Brooklyn, 1955.

2. Res1er, E. L., Jr, and W. R. Sears, "The Prospects of Magnetoaerodynamics," Journal of Aeronautical Sciences, 26, p. 235, Apri1, 1958.

3. Ericson, W., A. Maciulaitis and M. Falco, "Magnetoaerodynamic Drag and Flight Control," AIAA paper No. 65-630, August, 1965.

4. Wood, G. P., "The Electric Drag Forces on a Satellite in the Earth's Upper Atmosphere," Proceedings of the NASA - University Conference on the Science and Technology of Space Exploration,

5. Porter, R. W., and A. B. Cambe1, "Magnetic Coup1ing in F1ight Magnetoaerodynamics," AIAA Journal, Vo1. 5, No. 4, Apri1, 1967, PP. 803-805.

6. Bush, W. B., "Magnetohydrodynamic - Hypersonic Flow Past a Blunt Body," Journal of the Aero/Space Sciences, 25, p. 685-690,728, November, 1958.

7. Kemp, N. H., "On Hypersonic Blunt Body Flows with a Magnetic Field," Journa1 of the Aero/Space Sciences, 25, p. 405-407, June, 1958.

8. Freeman, N. C., "On the Flow Past a Sphere at Hypersonic Speed with a Magnetic Field," Iournal of the Aero/Space Sciences, 26, p. 670-672, Oct., 1959.

9. Kemp, N. H., "Author's Reply," Journal of the Aero/Space Sciences, 26, P. 672, October, 1959.

10. Ziemer, R. W., "Experimental Investigations in Magnetoaerodynamics," Journa1 of the American Rocket Society, Vo1. 29, No. 9, p. 642, 1959.

11. Wu, C. S., "Hypersonic Viscous Flow Near the Stagnation Point in the Presence of a Magnetic Field," Journal of the Aero/Space Sciences, 27, p. 882-893, 950, December, 1960.

12. Lykoudis, P. S., "The Newtonian Approximation in Magnetic Hypersonic Stagnation Point Flow," Journa1 of the Aero/Space Sciences, 28, pp. 541-546, Ju1y, 1960.

13. Lykoudis, P. S., "The Matching of the Viscid and Inviscid Regions for the Stagnation Magnetic Flow," Journal of the Aero/Space Sciences, 26, pp. 315-317, May, 1959. 
14. Pai, S. I., and E. T. Kornowski, "Stagnation Point Flow of Magnetized Blunt Body in Hypersonic Flow," Engineering Aspects of Magnetohydrodynamics, Edited by C. Mannal and N. Mathur, Columbia University Press, New York, 1962.

15. Levy, R. H., "A Simple MHD Flow with Hall Effect," AIAA Journa1, 1, p. 698-699, March, 1963.

16. Chuskin, P. I., "Magnetized Blunt Bodies in a Hypersonic Gas Flow," MHD, Vol. 1, No. 3, pp. 49, 55, July-September, 1965.

17. Smith, M. C., Schwimmer, H. S., and C. S. Wu, "MagnetohydrodynamicViscous and Inviscid Flow Near the Stagnation Point of a Blunt Body," AIAA Journa1, 3, pp. 1365-1367, July, 1965.

18. Smith, M. C. and C. S. Wu, "Magnetohydrodynamic Hypersonic Viscous F1ow Past a Blunt Body," AIAA Journal, 2, p. 963-965, May, 1964.

19. Porter, R. W. and A. B. Cambe1, "Comment on 'Magnetohydrodynamic Hypersonic Viscous and Inviscid Flow Near the Stagnation Point of a Blunt Body'," AIAA Journa1, 4, p. 952-953, May, 1966.

20. Smith, M. C., H. S. Schwimmer and C. S. Wu, "Author's Reply," AIAA Journal, 4, p. 953, May, 1966.

21. Levy, R. H. and H. E. Petschek, "Magnetohydrodynamically Supported Hypersonic Shock Layers," The Physics of Fluids, 6, P. 946-961, July, 1963.

22. Locke, E., Petschek, H. E., and P. H. Rose, "Experiments with Magnetica11y Supported Shock Layers," NASA CR-58823, August, 1964.

23. Levy, R. H. Gierasch, P. J. and D. B. Henderson, "Hypersonic Magnetohydrodynamics with and without a Blunt Body," AIAA Journal, 2, p. 2091-2099, December, 1.964.

24. Ericson, W. B., and A. Maciulaitus, "Investigation of Magnetohydrodynamic Flight Control," Journal of Spacecraft and Rockets, 1 , Pp. 283-289, May, 1964.

25. Porter, R. W., "Theoretical Aspects of Blunt Body Magnetoaerodynamics," Ph.D. Dissertation, Northwestern University, 1966.

26. Seeman, G. R., and A. B. Cambe1, "Observations Concerning Magnetoaerodynamic Drag and Shock Standoff Distance," Proceedings of the National Academy of Sciences, 55, pp. 457-465, March, 1966.

27. Nowak, R. J., Kranc, S., Porter, R. W., Yuen, M. C, and A. B. Cambe1, "Magnetogasdynamic Re-Entry Phenomena," Journal of Spacecraft and Rockets, Vo1. 4, No. 11, pp. 1538-1542, December, 1967. 
28. Cambe1, A. B., "Phenomenological Aspects of Magnetogasdynamic ReEntry," Invited General lecture, 10th Midwestern Mechanics Conference, Colorado State University, August, 1967.

29. Cambe1, A. B., Plasma Physics and Magnetofluidmechanics, McGraw-H111 Book Co., Inc., New York, 1963.

30. Scinlicting, H., Boundary Layer Theory, McGraw-Hi11, New York, 1960.

31. Seemanu, G. R., "Experimental Aspects of Magnetoaerodynamic Drag," Ph.D. Dissextation, Northwestern University, 1963.

32. Addock, B. D., "Temperature Measurements on a Subatmospheric Argon Plasma Jet," Journal Quantitative Spectroscopy and Radiative Transfer, Vo1. 7, pp. 385-400, 1957.

33. Grewa1, M. S. and L. Talbot, "Shock Wave Structure in a Partialiy Ionized Gas," Journal of Fluid Mechanics, Vol. 16, pp. 473-574, 1963.

34. Arave, R. J., and 0. A. Huseley, "Aerothermodynamic Properties of High Temperature Argon," Boeing Document D2-11238, February, 1962.

35. Keenan, J. H., and J. Kaye, Gas Tables, Wiley, New York, 1948.

35. von Engle, A., Ionized Gases, Oxford Claredon Press, England.

37. Spitzer, Jyman, Jr., Physics of Fully Ionized Gases, Interscience Publishers, Inc., New York, 1956.

38. Chen, C. J., "Anommalous Diffusion and Instabilities of an Argon Plasma in a Strong Magnetic Field," Technical Report No. 32-695, Jet Fropulsion Laboratories, Lecember, 1964.

39. Bates, D. R., Kingston, A. E., McWirther, "Recombination Between Electrons and Atomic Ions (Part I)," Proc. Roya1 Soc., Vo1. 267A, p. 297, May, 1967.

40. Arney, G. D., and A. B. Pailey, "Effect of Temperature on Pressure Measurements," AIAA Journal, Vol. 1, No. 12, pp. 2863-2864, Dec., 1963.

41. Jaffrin, M. Y., "Shock Structure in a Partia11y Ionized Gas," Physics of Fluids, Vo1. 8, No. 4, p. 606-625, Apri1, 1965.

42. Chen, C. J., "Spectroscopic Measurement of a Detached Shock Wave in an Arc-Heated Flasma Jet," JPL Space Programs Sumnary, VoI. IV, No. $37-32$.

43. Sonnin, A.A., "The Behavior of Free Molecular Cylindrical Probes in Supersonic Flows, and Their Application to the Study of the Blunt Body Stagnation Layer," U'IIAS Report No. 109, August, 1965. 
44. Christiansen, W. H., "Study of Shock Waves in a Non-Equilibrium Plasma," Physics of Fluids, Vol. 10, No. 12, pp. 2586̈-2595.

45. Pipkin, A. C., "Diffuston from a Slightly Ionized Region in a Uniform Flow," Physics of Fluids, Vo1. 4, No. 10, p。1298, 1302, October, 1961 .

46. Probestein, R. F., and N.H. Kemp, "Viscous Aerodynamic Characteristics in Hypersonic Rarefled Gas Flow," Journal of the Aero/Space Sciences, pp. 174-191, March, 1960.

47. Knopp, C. F., C. F. Gottschlich and A. B. Cambe1, "A Spectroscopic Technique for the Measurement of Temperature in Transparent P1asma," AFOSR $-1100,1961$.

48. Wilson, R., "The Spectroscopy of Non-Therma1 p1asmas, " Journal of Quantitative Spectroscopy and Radiative Transfer, Vol. 2, No. 4, Pp. 477, 490, October-December, 1962 .

49. Adock, B. D. and W.E.G. Plumbtree, "On Excitation Temperature Measure-. ments in Plasma-Jet and Transition Probabilities for Argon Lines," Journal of Quantative Spectroscopy and Radiative Transfer, Vol. 4, Pp. 29-39, 1964.

50. Chen, C. J., "Valid Conditions for the Kramers-Unsold Continuum Theory in a Non-Equilibrium Plasma," Technical Report 32-707, Jet Propulsion Laboratory, December, 1964.

51. Thornton, J. A., "A Spectroscopic Study of the Argon Plasmas Generated in a Conical Type Electromagnetic Shock Tube,: Ph.D. Dissertation, Northwestern University, June, 1963.

52. olsen, H. N., "Partition Function Cut-off and Lowering of the Ionization Potential in an Argon Plasma," Physical Review, Vo1. 12.4, No. 6, 1703, December, 1961.

53. De Vos, J. C., and G.A.W. Rutgers, "Relation Between Brightness Temperature, True Temperature and Color Temperature of Tungsten," Physica, Vo1. 20, p. 715, 1954.

54. Harrison, G. R., Lord, R. C., Loofborrow, J. R., Practical Spectroscopy, Prentice Hal1, New York, 1948.

55. Porter, R. W., "Numerica1 Solutions for Local Emission. Coeffirfents in Axisymetric Self-Absorbed Sources," SIAM Review 6, 228-241, July, 1967.

56. Bailey, A: B., and Sims, W. H., "Shock Detachment Distance for Blunt Bodies in Argon at Low Reynolds Number," AIAA Journal, Vo1. 1, No. 1, December, 1963. 
57. Brewer, I. E. and McGregor, "The Radiative Decay of Metastable Argon Atoms in a Low Density Argon Plasma Stream, Physics of Fluids, 5, 1485,1962 .

58. Fay, J. A., Report AMP 71, Avco Everett Research Laboratory, 1962.

59. Petschek, H., and S. Byron, "Approach to Equilibrium Ionization Behind Strong Shock Waves in Argon," Annals of Physics, 1, Pp. 270$315,1957$.

60. Chen, C. J., "Partition of Recombination Energy in the Decaying RareCas P1asmas," Physical Review, 163, p. 1-7, 1967.

61. Baird, D. C., Experimentation: An Introduction to Measurement Theory and Experiment Design, Prentice Hal1, Inc. 1962.

62. Cheng, H. K., "The Blunt Body Problem in Hypersonic Flow at Low Reynolds Number," Corne11 Aeronautical Report, No. AF 1285-A-10, June, 1963.

63. Kranc, S., Porter, R. W. and A. B. Cambel, "Electrodeless Magnetogasdynamic Power Generation During Entry," Journal of Spacecraft and Rockets, Vo1. 4 and 6, Pp. 813-815, June, 1967. 
TABLE I: Literature Survey

\begin{tabular}{|c|c|c|c|c|c|c|c|c|c|}
\hline & eference & Approach & Rem. & $s$ & $\mathrm{C}_{\mathrm{H}}$ & $R_{e}$ & $\begin{array}{c}\text { Shock } \\
\text { Standoff }\end{array}$ & $\begin{array}{r}\text { Total } \\
\text { Drag }\end{array}$ & Other \\
\hline 6. & $\begin{array}{l}\text { - Bush } \\
\text { (1958) }\end{array}$ & Theoretical & Arb. & Arb. & 0 & & yes & no & $\begin{array}{l}\text { Calculated pressure } \\
\text { distribution }\end{array}$ \\
\hline 7. & $\begin{array}{l}\text { Kemp } \\
(1958)\end{array}$ & Theoretical & 0 & $\mathrm{sm}$. & 0 & & yes & no & $\begin{array}{l}\text { Specified radial } \\
\text { field }\end{array}$ \\
\hline 10. & $\begin{array}{l}\text { Ziemer } \\
\text { (1969) }\end{array}$ & Experimental & 0 & Arb. & $?$ & $?$ & yes & no & \\
\hline 12. & $\begin{array}{l}\text { Lykoudis } \\
(1960)\end{array}$ & Theoretical & 0 & Sm. & 0 & & yes & no & $\begin{array}{l}\text { Assumed pressure } \\
\text { distribution }\end{array}$ \\
\hline 16. & $\begin{array}{l}\text { - Chuskin } \\
(1965)\end{array}$ & Theoretical & 0 & Moderate & 0 & Arb. & yes & no & \\
\hline 20. & $\begin{array}{l}\text { Levy } \\
\frac{\text { et al. }}{(1964)}\end{array}$ & Theoretical & 0 & Large & 0 & & yes & no & \\
\hline 22 . & $\begin{array}{l}\text { Porter } \\
(1966)\end{array}$ & Theoretical & Arb. & Arb. & Arb. & Arb. & yes & yes & $\begin{array}{l}\text { Calculated individual } \\
\text { components of drag }\end{array}$ \\
\hline 23 . & $\begin{array}{l}\text { Seeman \& } \\
\text { Cambel } \\
(1966)\end{array}$ & Experimental & Sm. & Sm. & 0 & $0(1000)$ & yes & yes & \\
\hline 24. & $\begin{array}{l}\text { Nowak } \\
\frac{\text { et a1. }}{(1967)}\end{array}$ & $\begin{array}{l}\text { Experimental } \\
\text { Theoretical }\end{array}$ & Sm. & Moderate & Moderate & $c(100)$ & yes & yes & \\
\hline
\end{tabular}


TABLE II: Spectroscopic Information

\begin{tabular}{|c|c|c|c|c|c|c|}
\hline$\lambda(\AA)$ & $\mathrm{g}_{\mathrm{n}}$ & $A_{n}\left(x 10^{-5} \sec ^{-1}\right)$ & $E_{n(e v)}$ & $w^{\prime}(m m)$ & $\mathrm{c}_{\mathrm{AL}}$ & $\frac{\lambda}{\mathrm{gAC}}$ \\
\hline 4333 & 5 & 4.9 & 14.69 & .200 & 0.67 & 2.64 \\
\hline 4345 & 3 & 2.8 & 14.68 & .200 & 0.67 & 7.75 \\
\hline 4510 & 1 & 10.2 & 14.57 & .225 & 0.77 & 5.79 \\
\hline 5650 & 1 & 19 & 15.10 & .375 & 1.0 & 3.00 \\
\hline 5606 & 3 & 15 & 15.12 & .300 & 1.0 & 1.36 \\
\hline 4300 & 5 & 3.4 & 14.5 & .4000 & 0.63 & 4.03 \\
\hline
\end{tabular}


TABLE III: Empirical Equations for the Data

Date

1. Total Drag

2. Lorentz Force

3. Pressure Force

4. Shock Standoff
Fit

Eq. (6.9)

Eq. (6.4) $\quad \frac{\mathrm{L}_{\mathrm{D}}}{\mathrm{D}_{\mathrm{O}}} \times 100 \%=.93 \mathrm{~S} \%$

Eq. (6.5) $\frac{\Delta P_{D}}{D_{o}} \times 100 \%=-.24 \mathrm{~s} \%$

Eq. (6.10) $\frac{\Delta_{n}-\Delta_{o}}{R_{b}}=.48 \times 10^{-2} \mathrm{~s}$

Equation*
Standard Deviation ( 8$)$

$2.13 \%$

$3.5 \%$

$1.26 \%$

.0117

* In all cases: $\quad \mathrm{S}=2.0 \mathrm{~B}_{\mathrm{o}}^{2} ; \quad \mathrm{C}_{\mathrm{H}}=0.8 \mathrm{~B}_{\mathrm{o}} ; \mathrm{C}_{\mathrm{H}}=.57 \sqrt{\mathrm{S}}$

99 


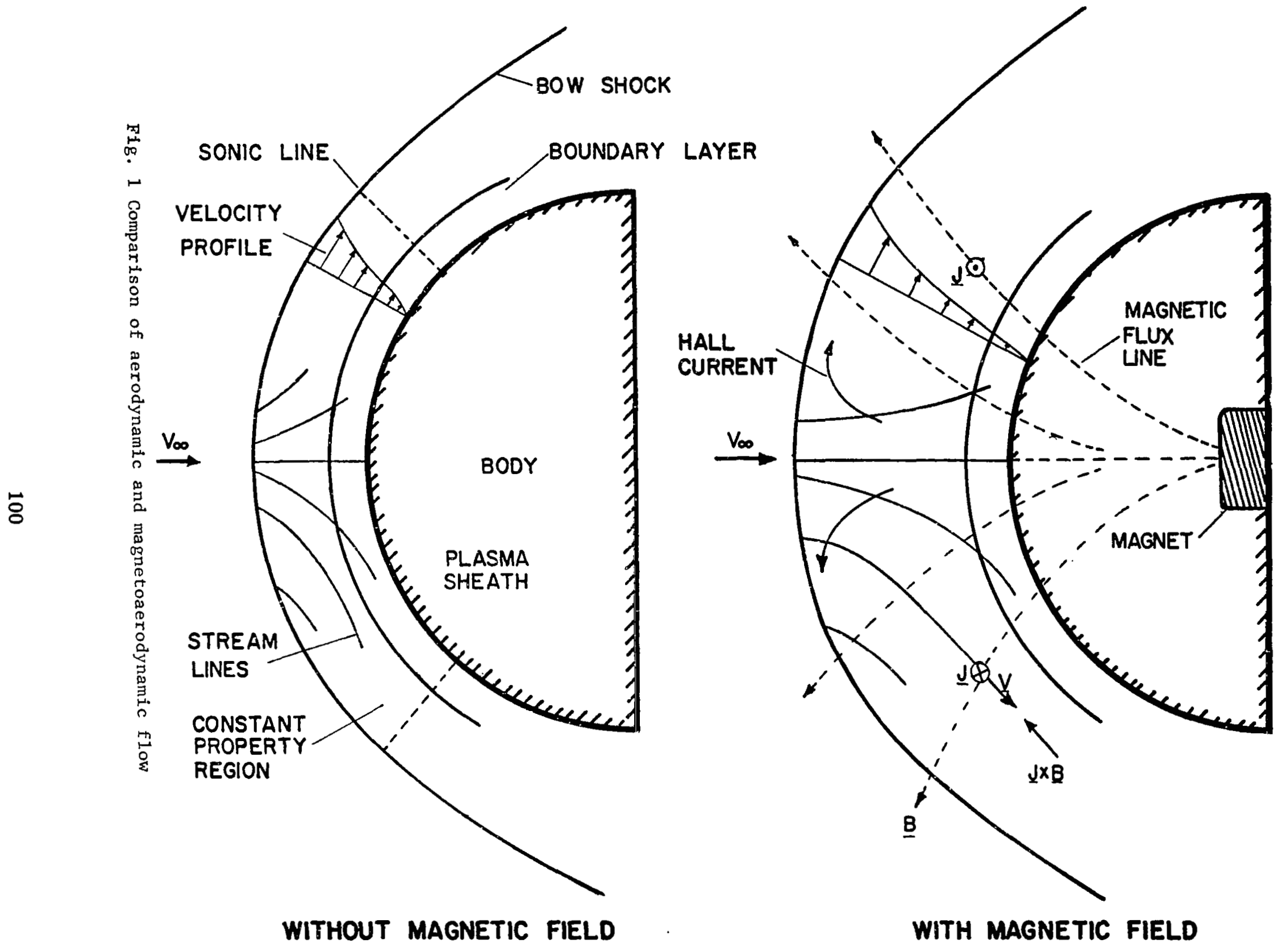




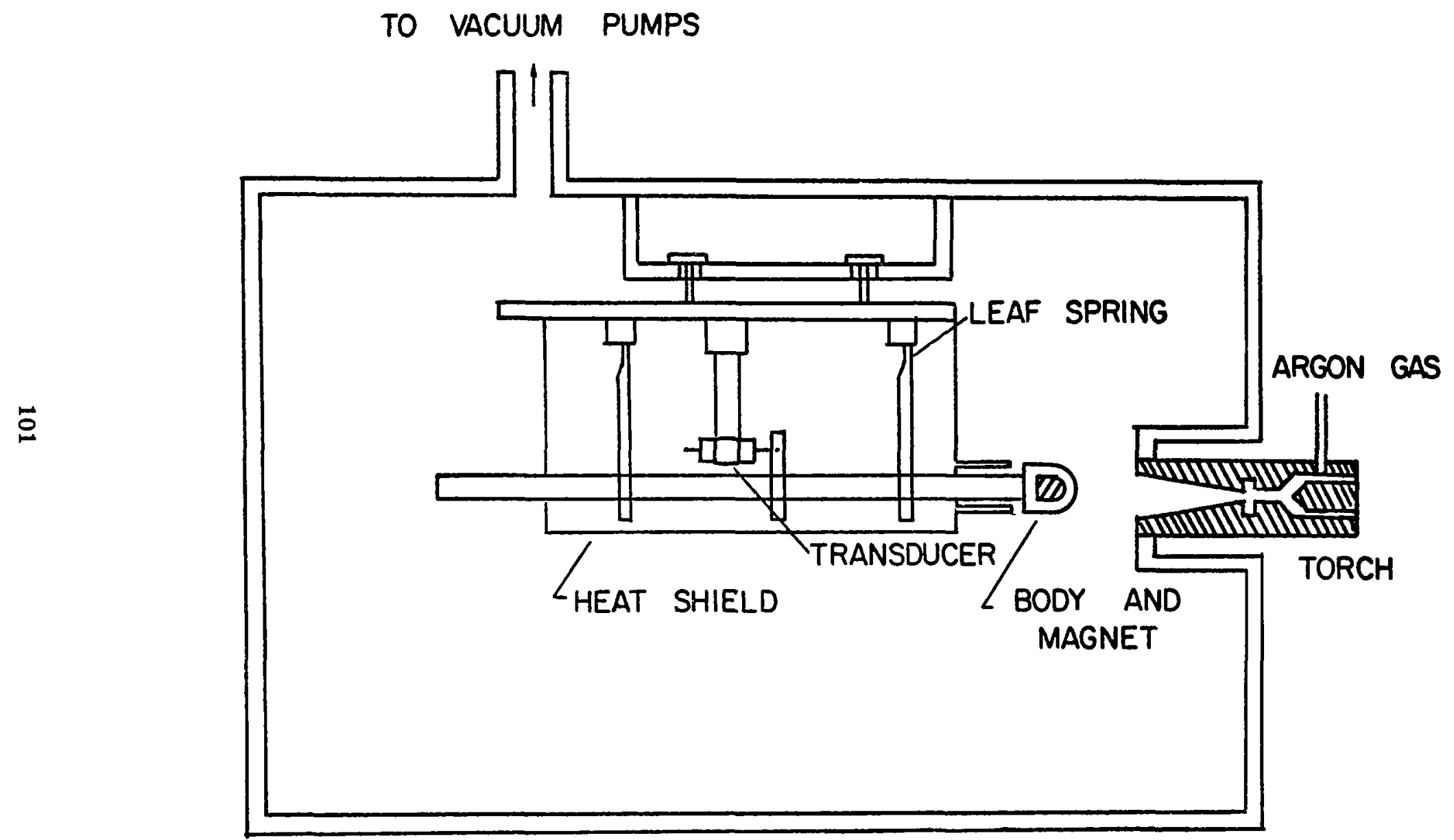

F18. 2 Schematic of facility 


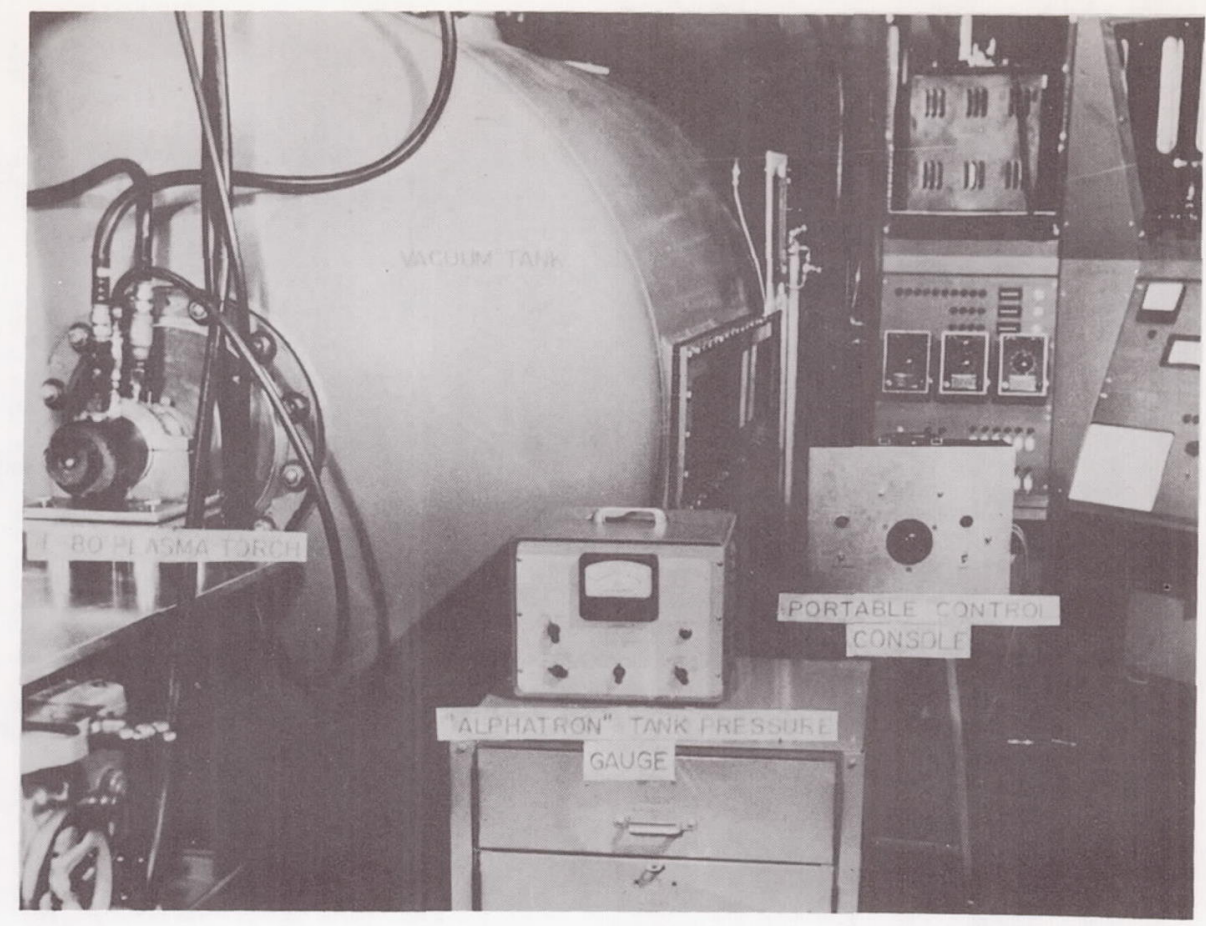

Fig. 3A Photograph of facility showing operating console

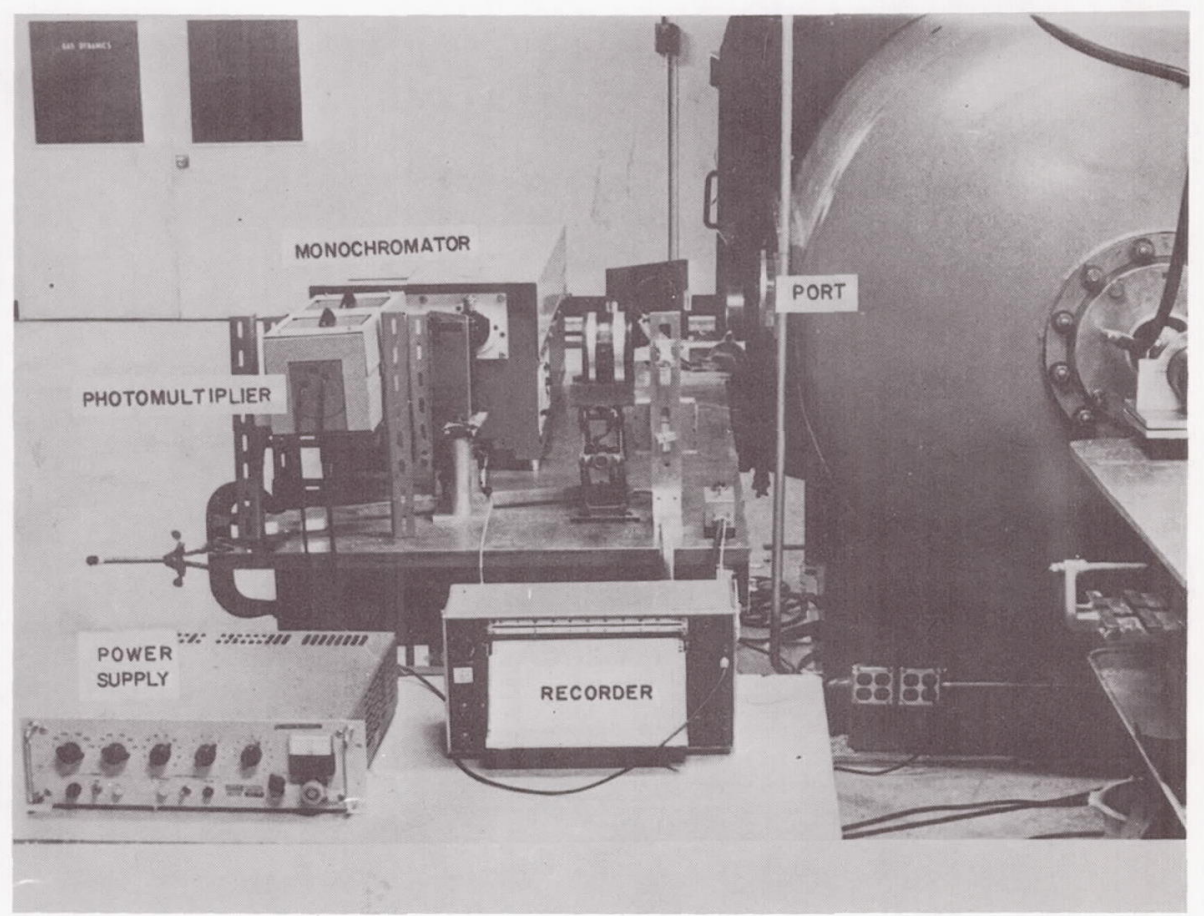

Fig. 3B Photograph of facility showing spectroscopic apparatus 


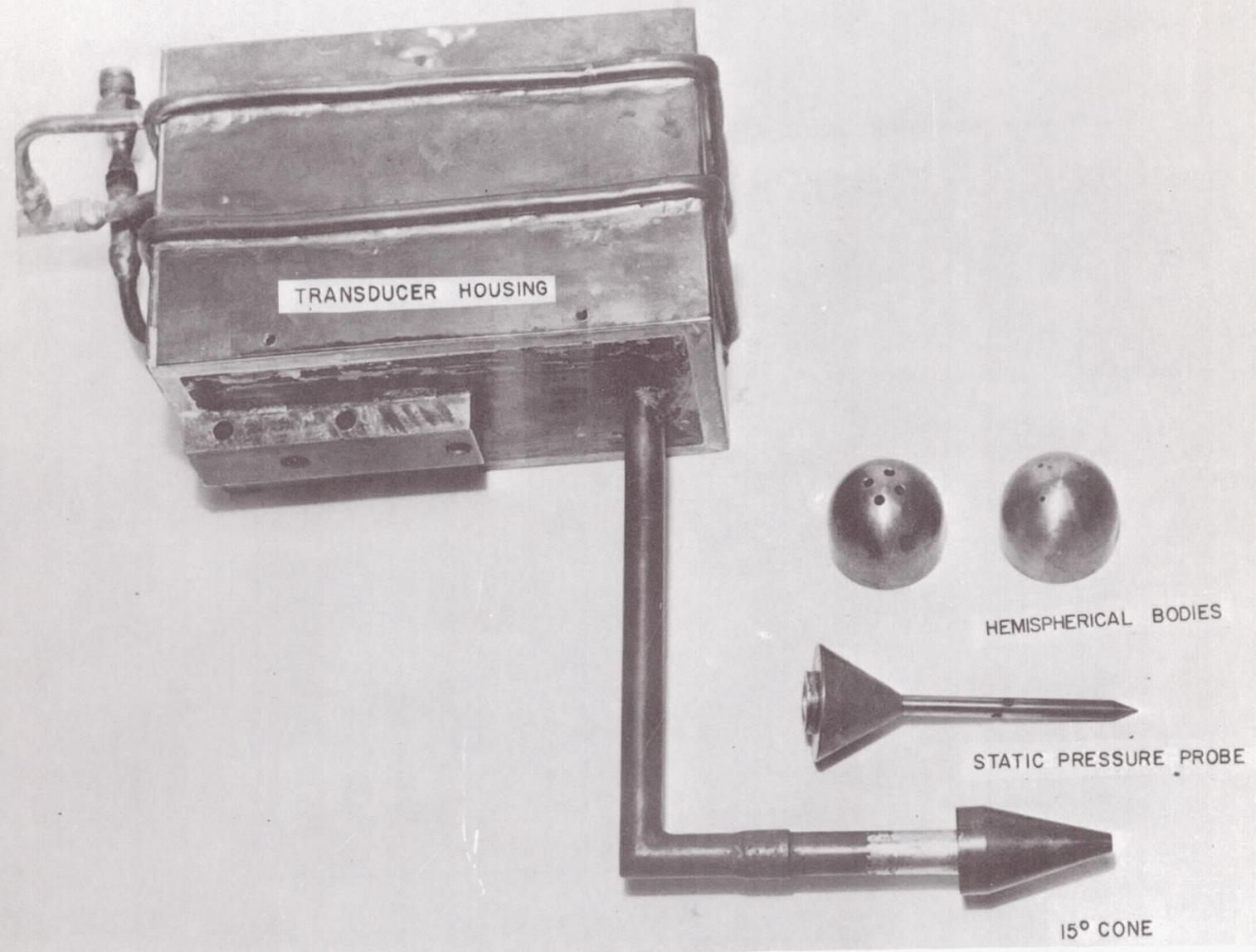

Fig. 4 Photograph of pressure probe and various heads 


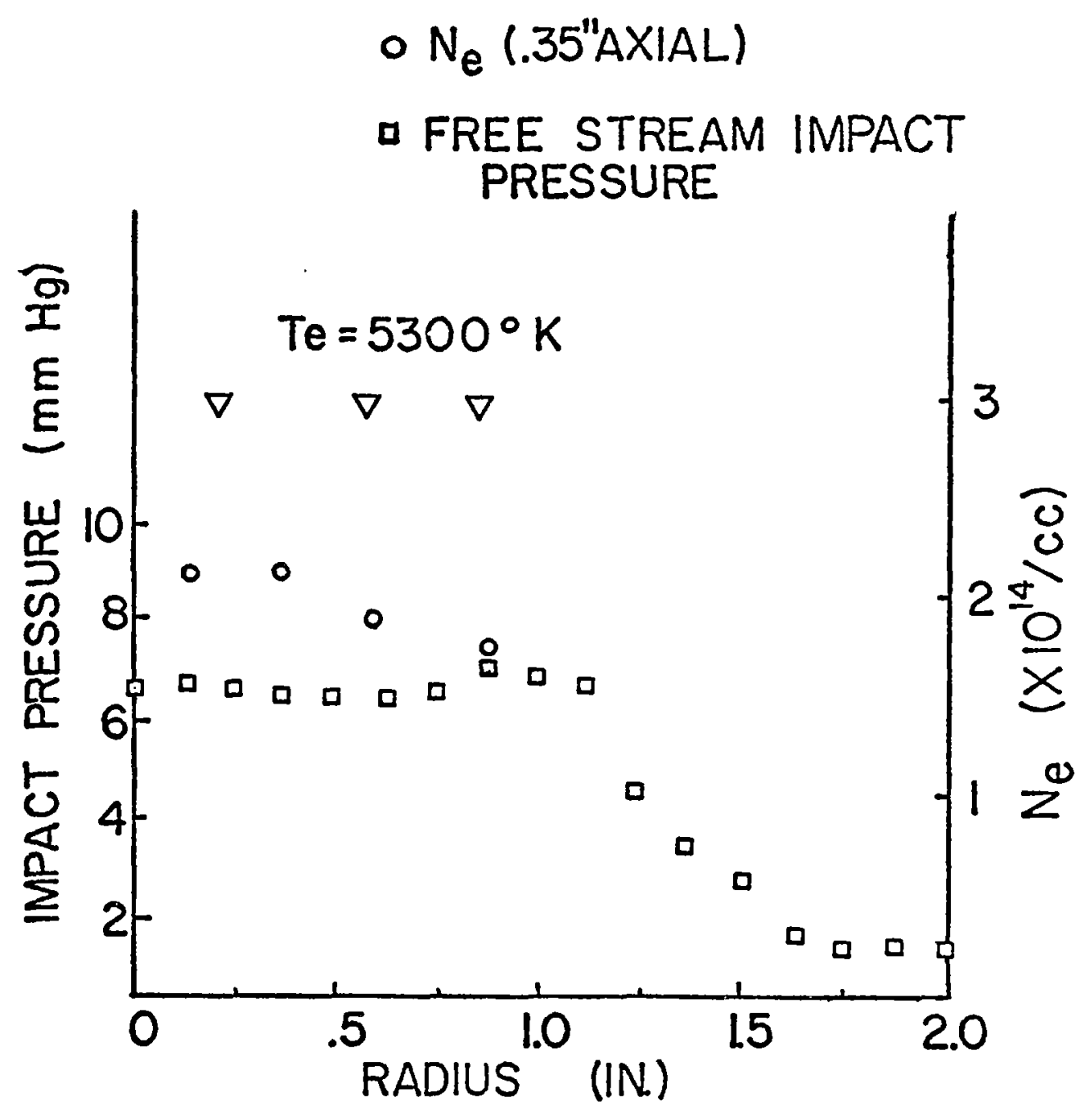

Fig. 5 Freestream property profiles 


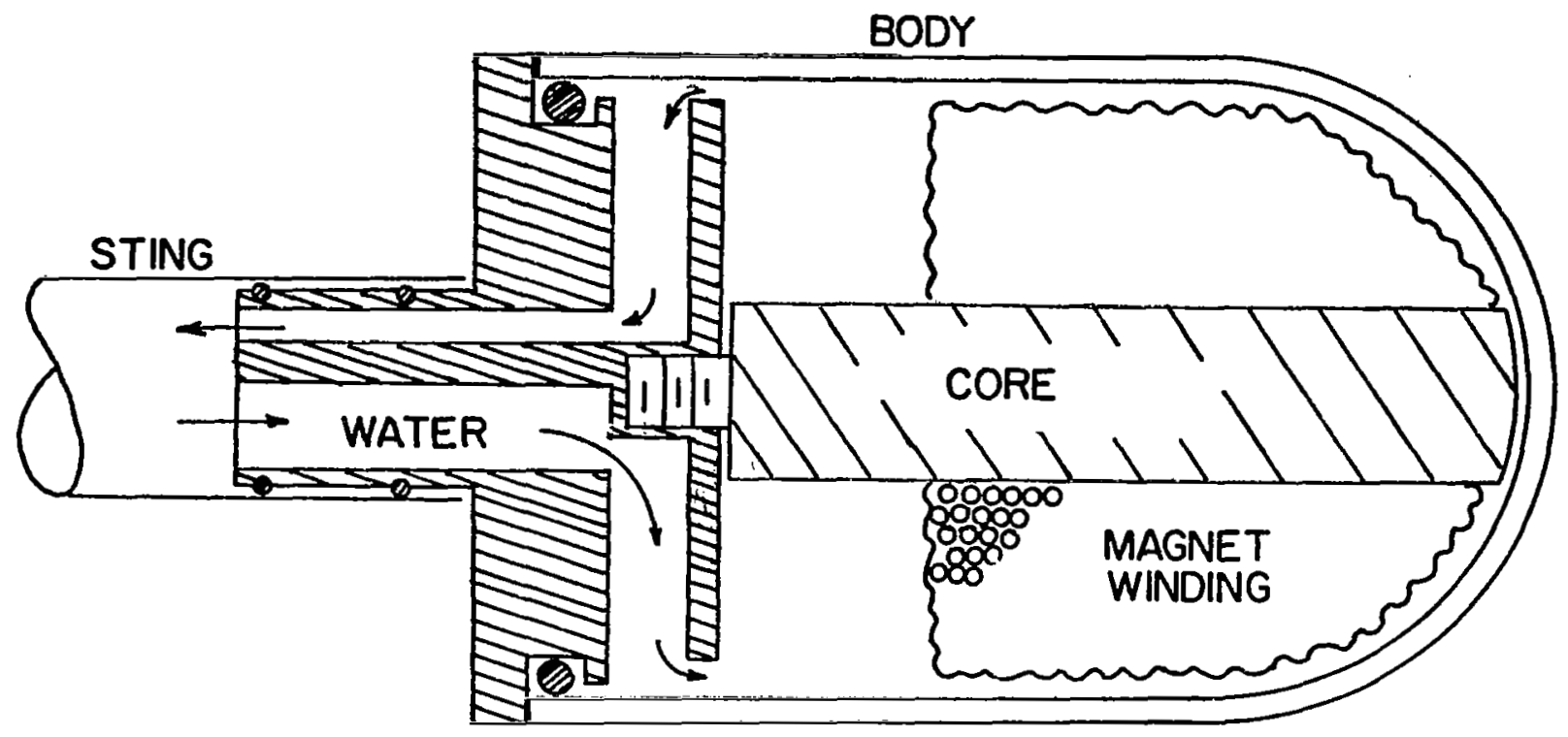

INTERIOR DETAIL - BODY AND MAGNET

(DOUBLE SIZE)

Fig. 6 Interior detail body and magnet 


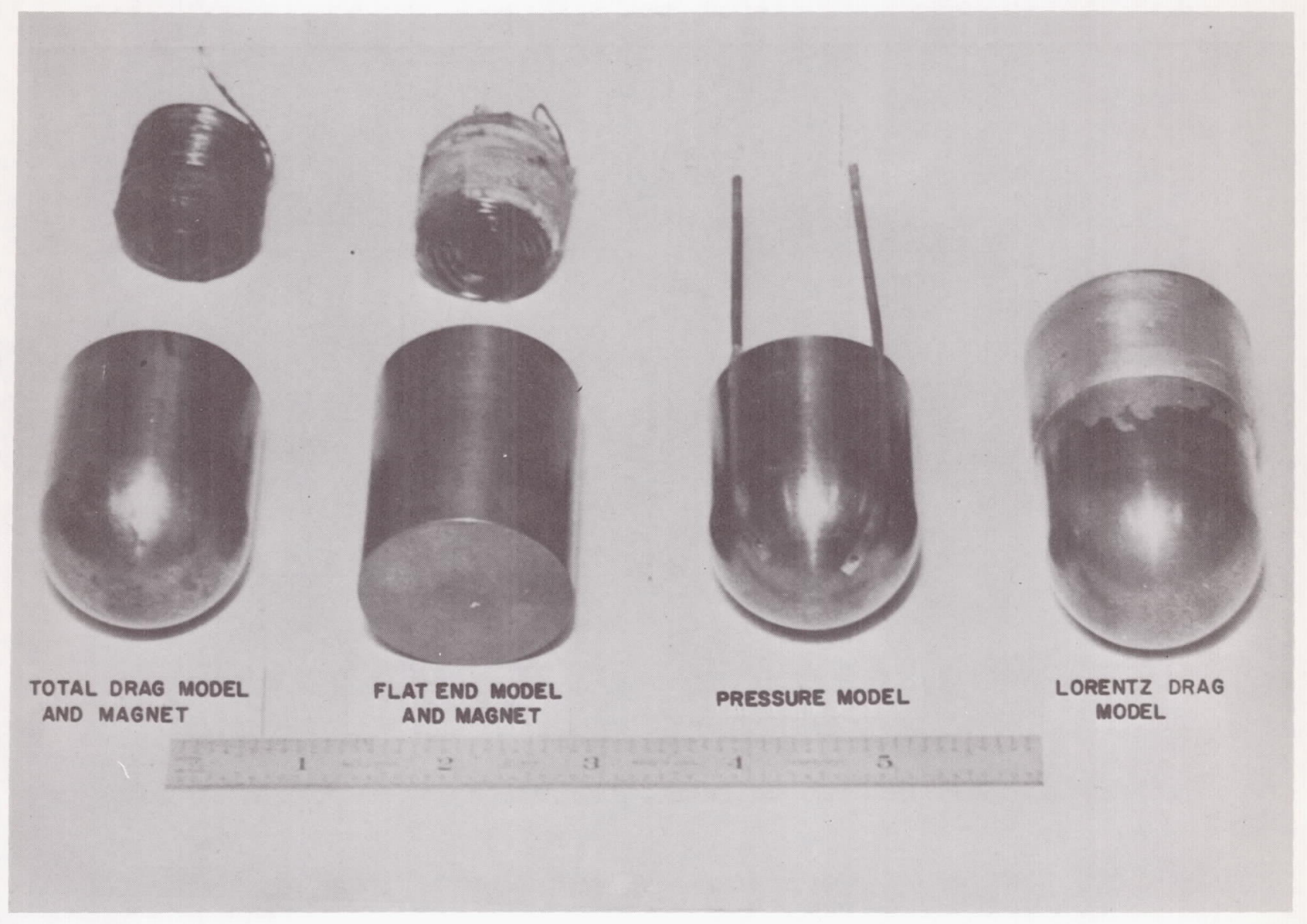

Fig. 7 Photograph of bodies and magnets used in magnetoaerodynamic drag measurements 


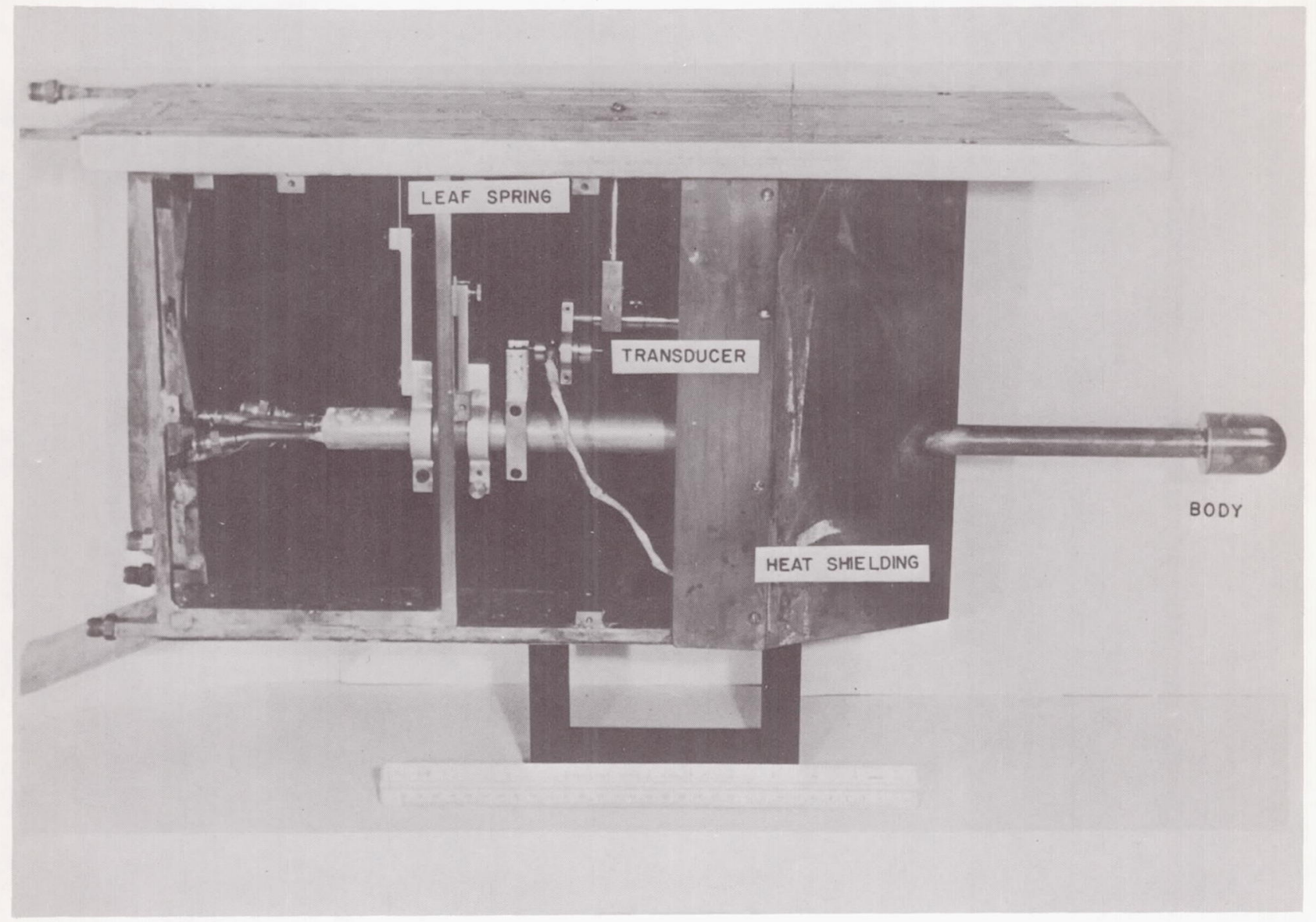

Fig. 8 Photograph of drag measuring apparatus 


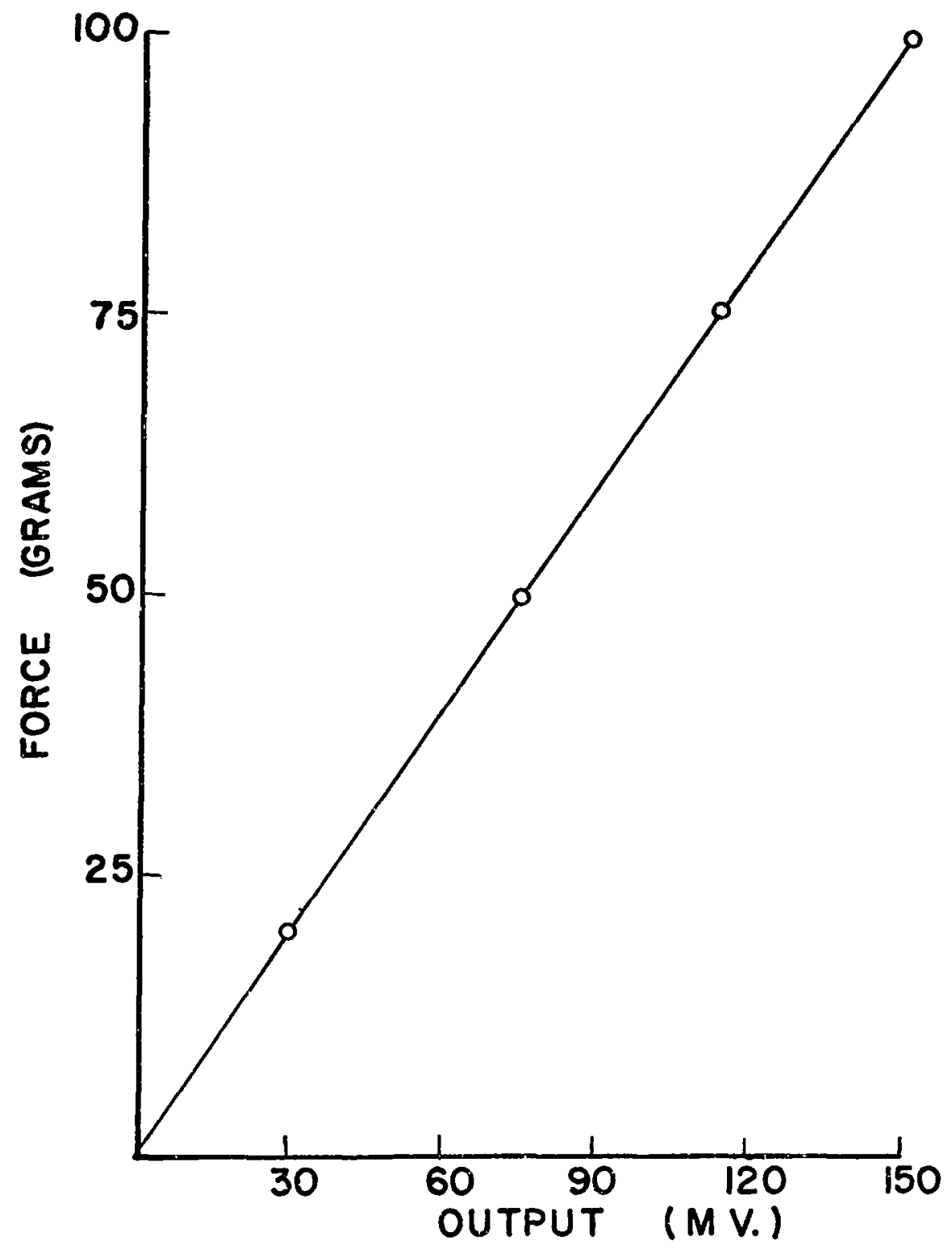

FIg. 9 Typical force calibration for drag tests 


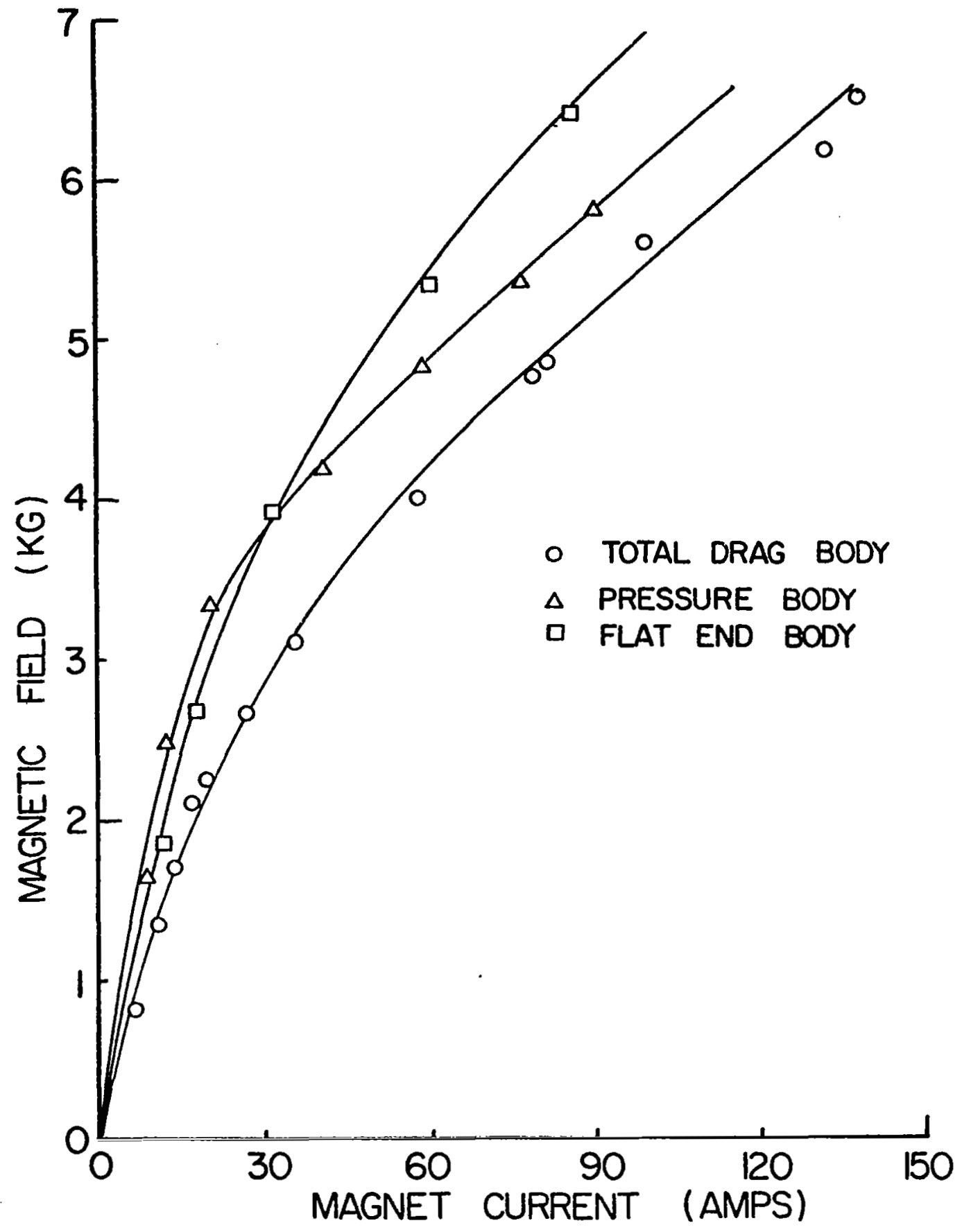

Fig. 10 Sample magnetic fleld strengths 


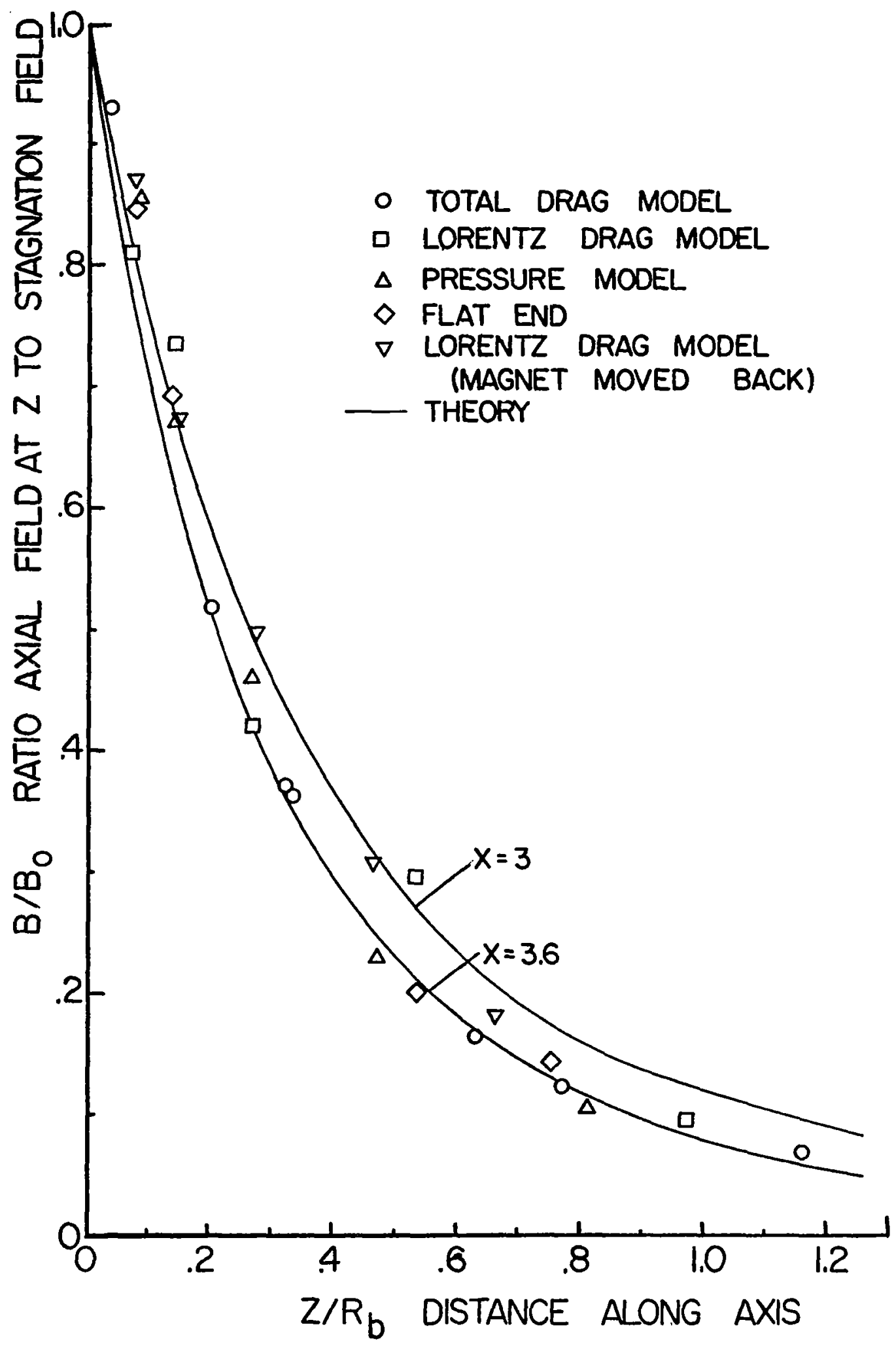

Fig. 11 Field drop off with axial distance 


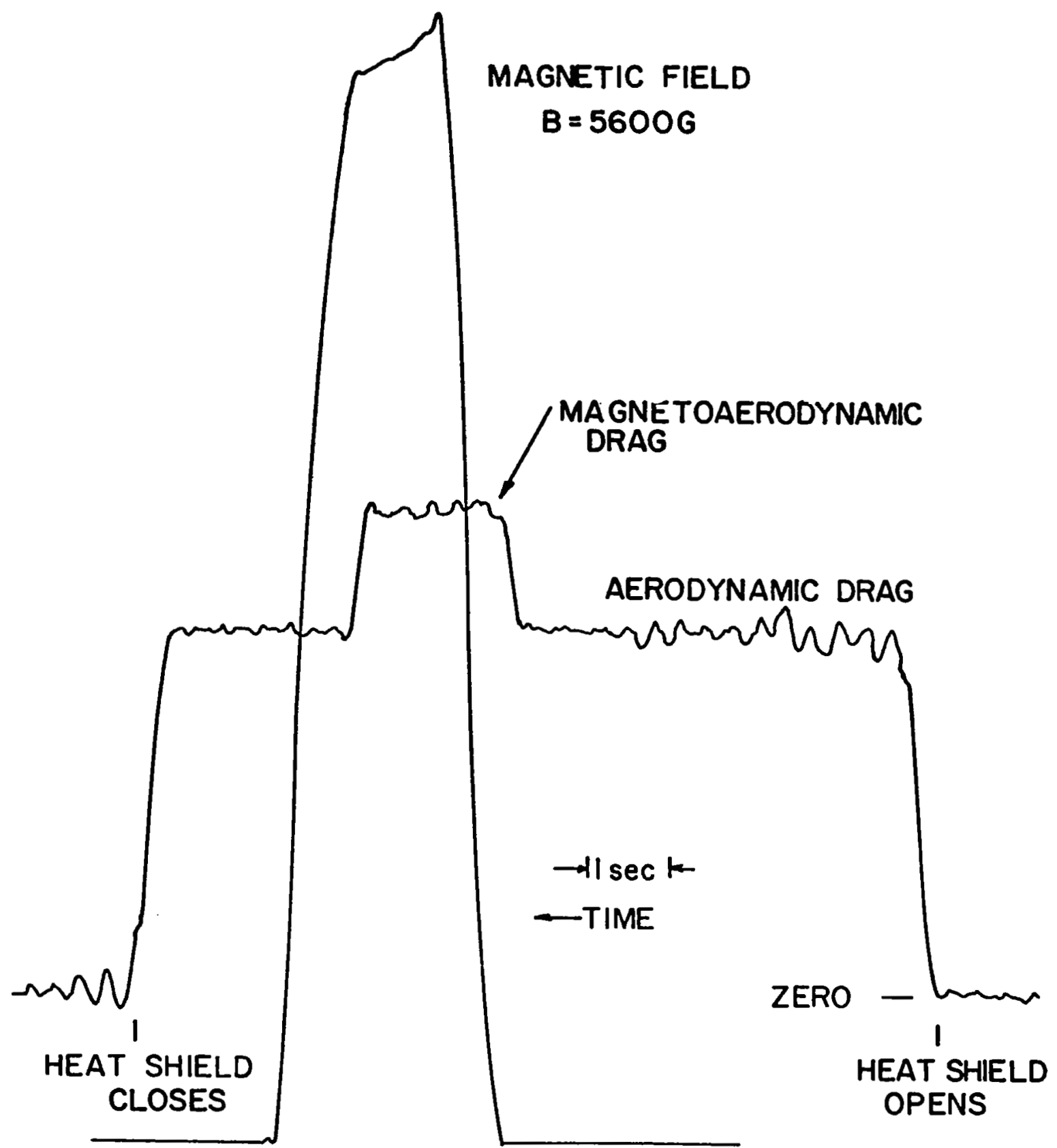

F1g. 12 Typical trace of drag test. (The apparant difference ( 1/2 sec) between the initial rises of both traces is due to mechanical crossover. Rises are actually simultaneous.) 


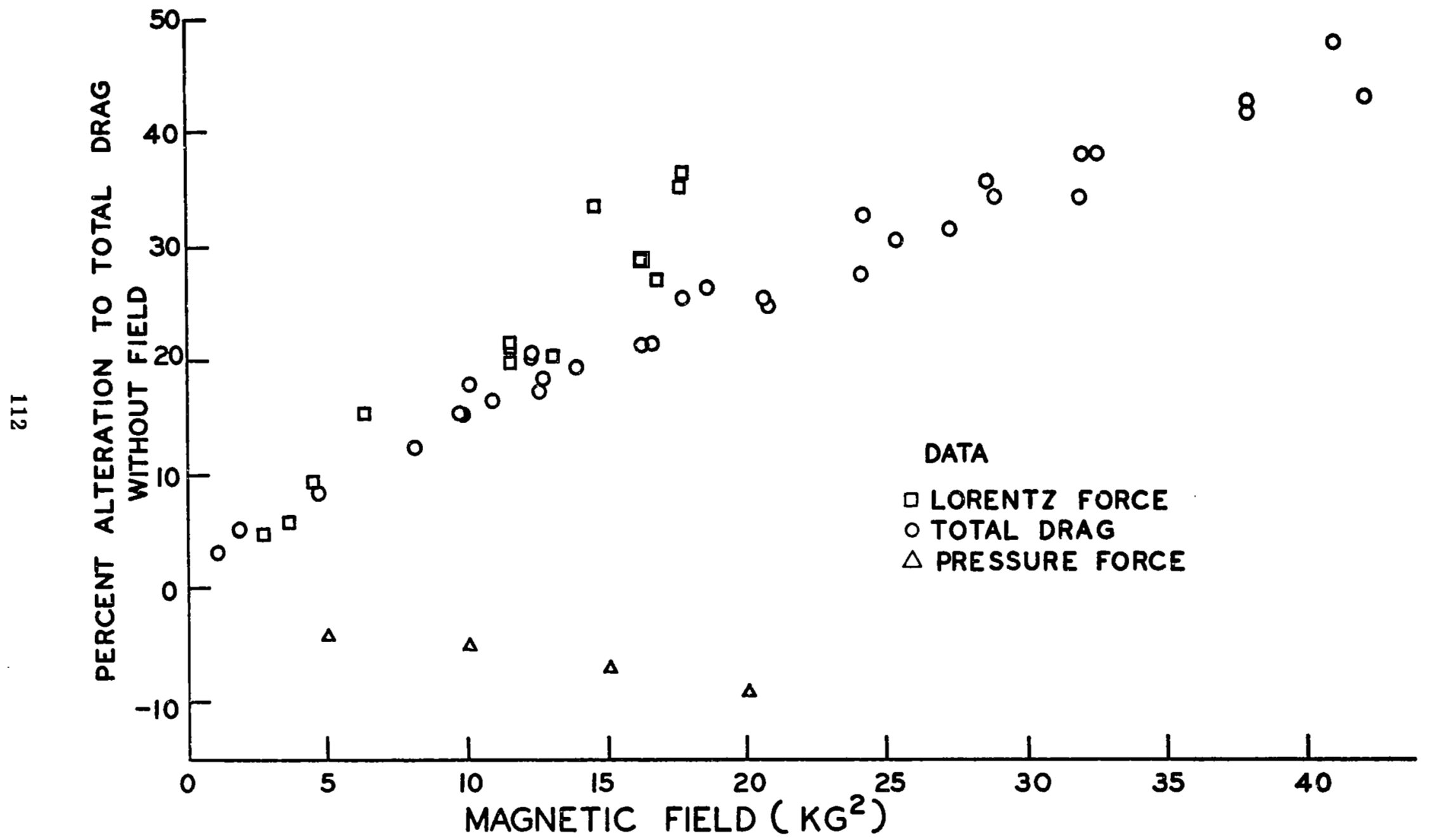

Fig. 13 Alteration to total drag and components with magnetic field 


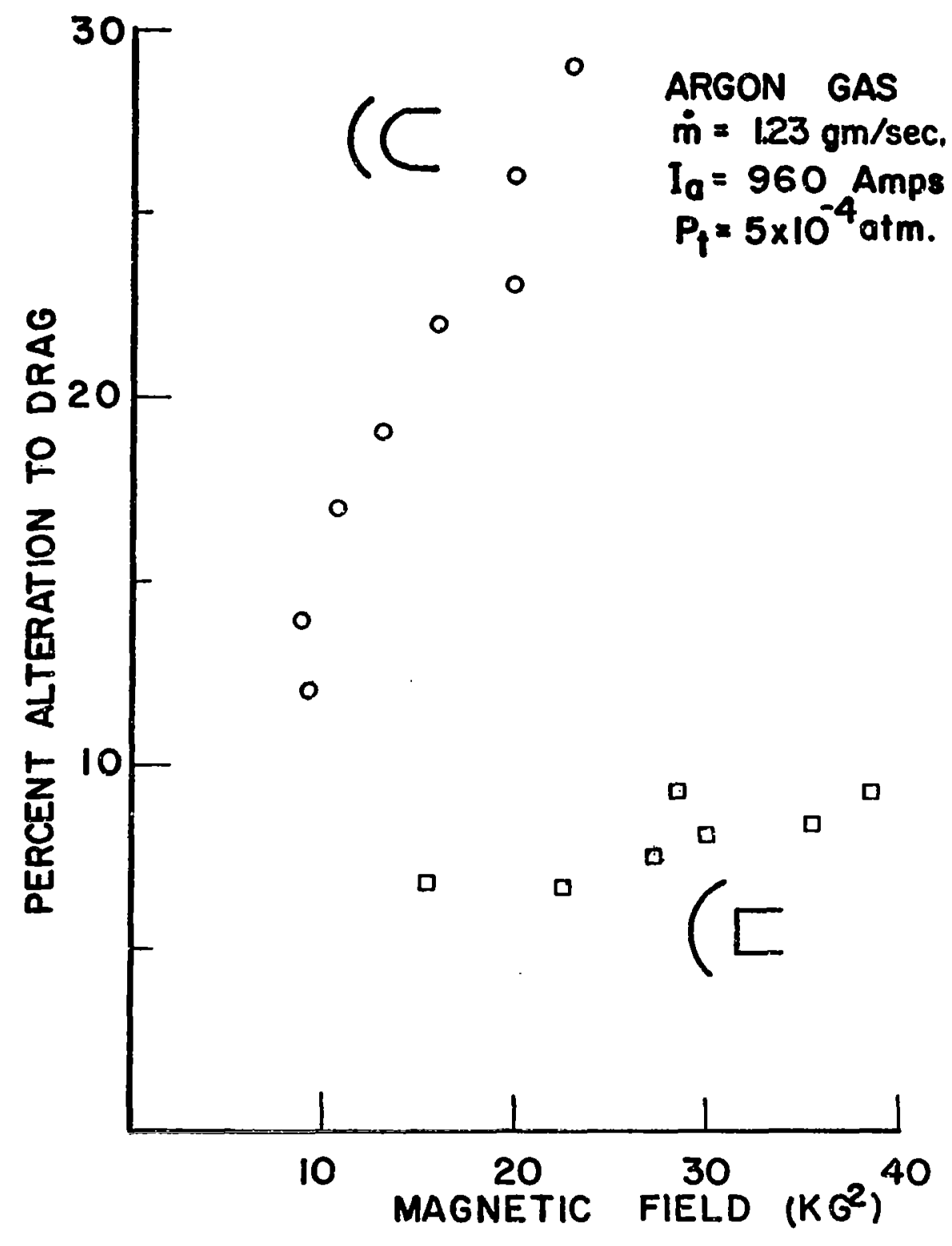

Fig. 14 Comparison of total drag alteration for two body shapes 


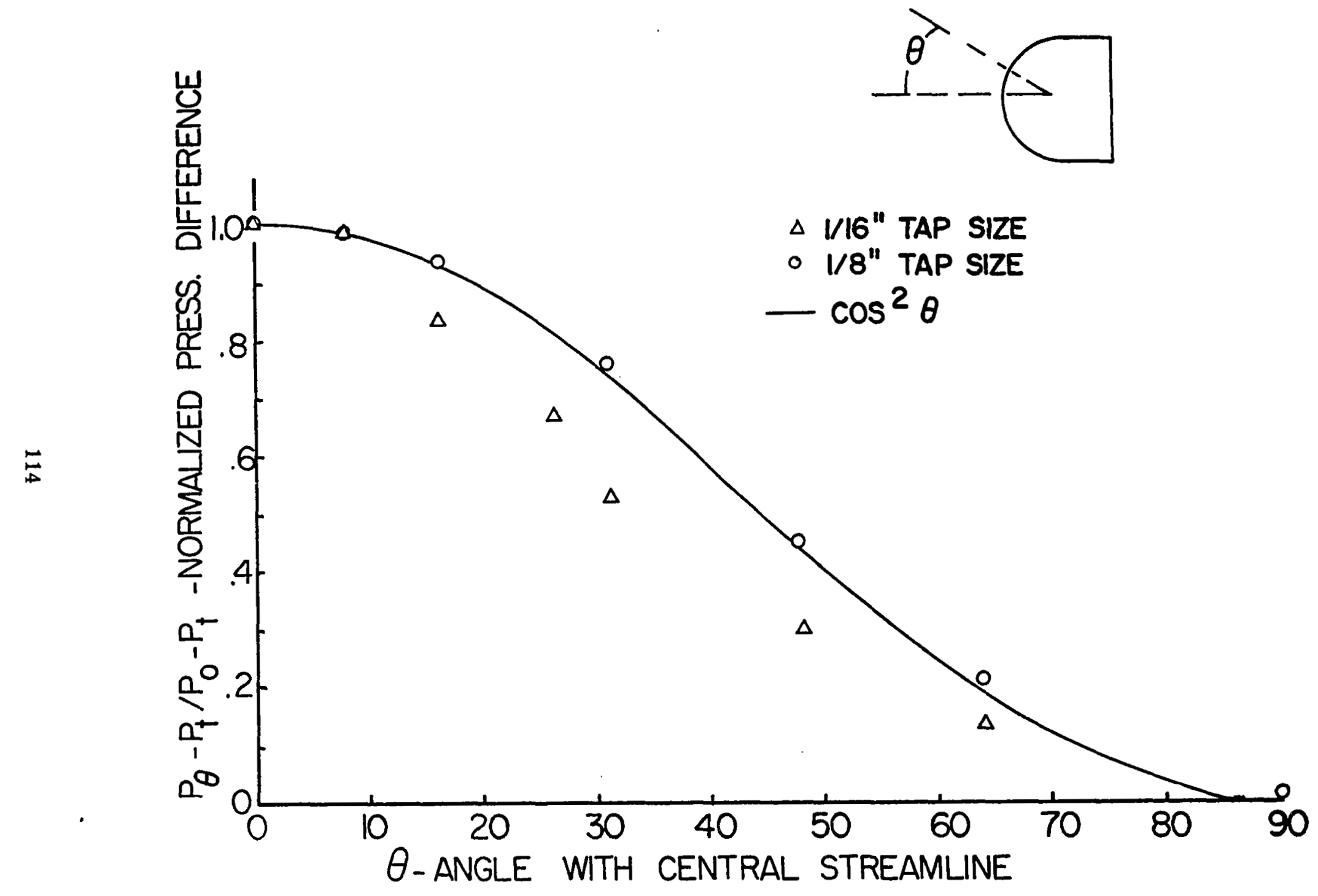

Fig. 15 Pressure distribution on body for two tap sizes 


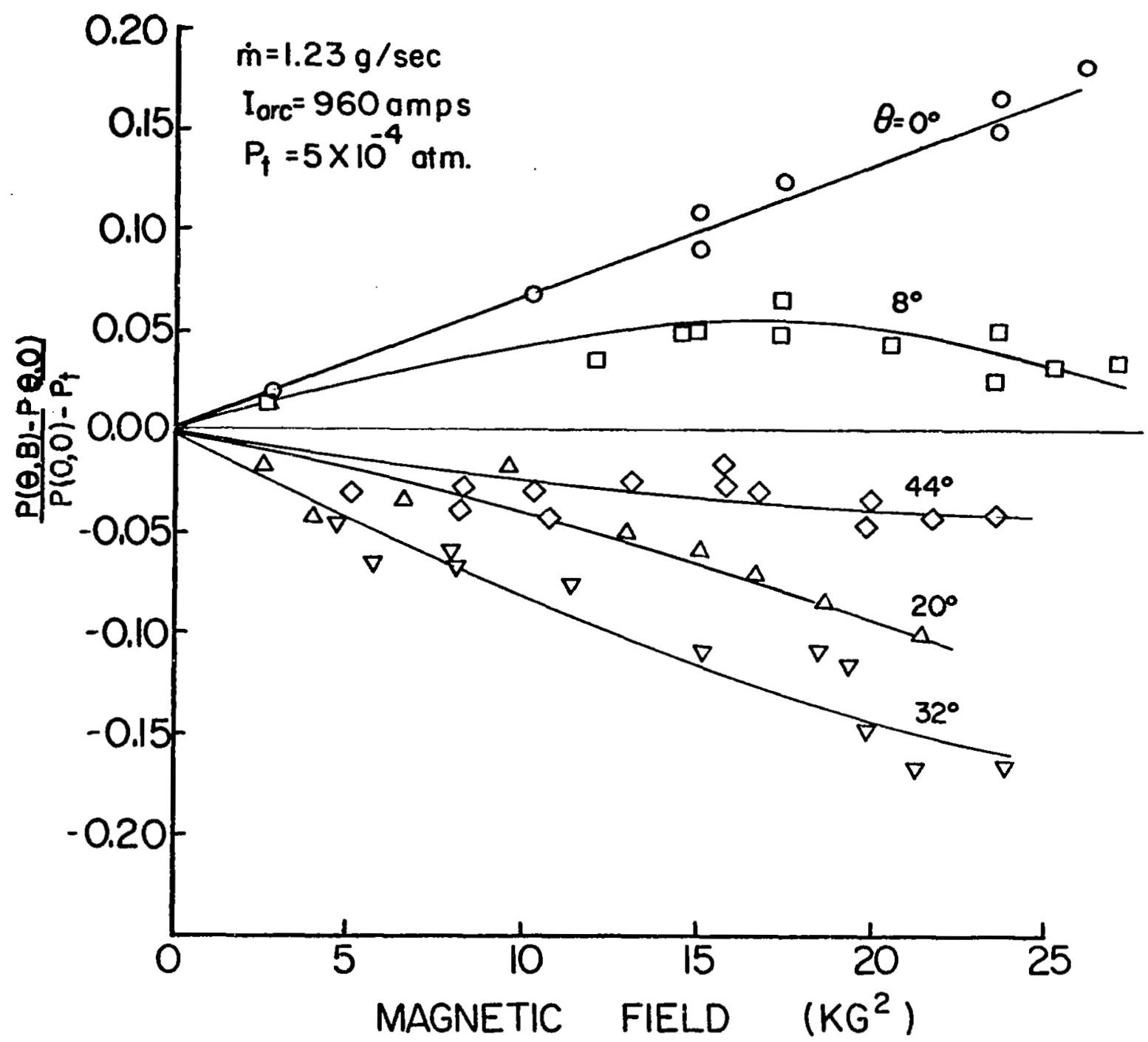

FIg. 16 Pressure change at various angles as a function of magnetis 


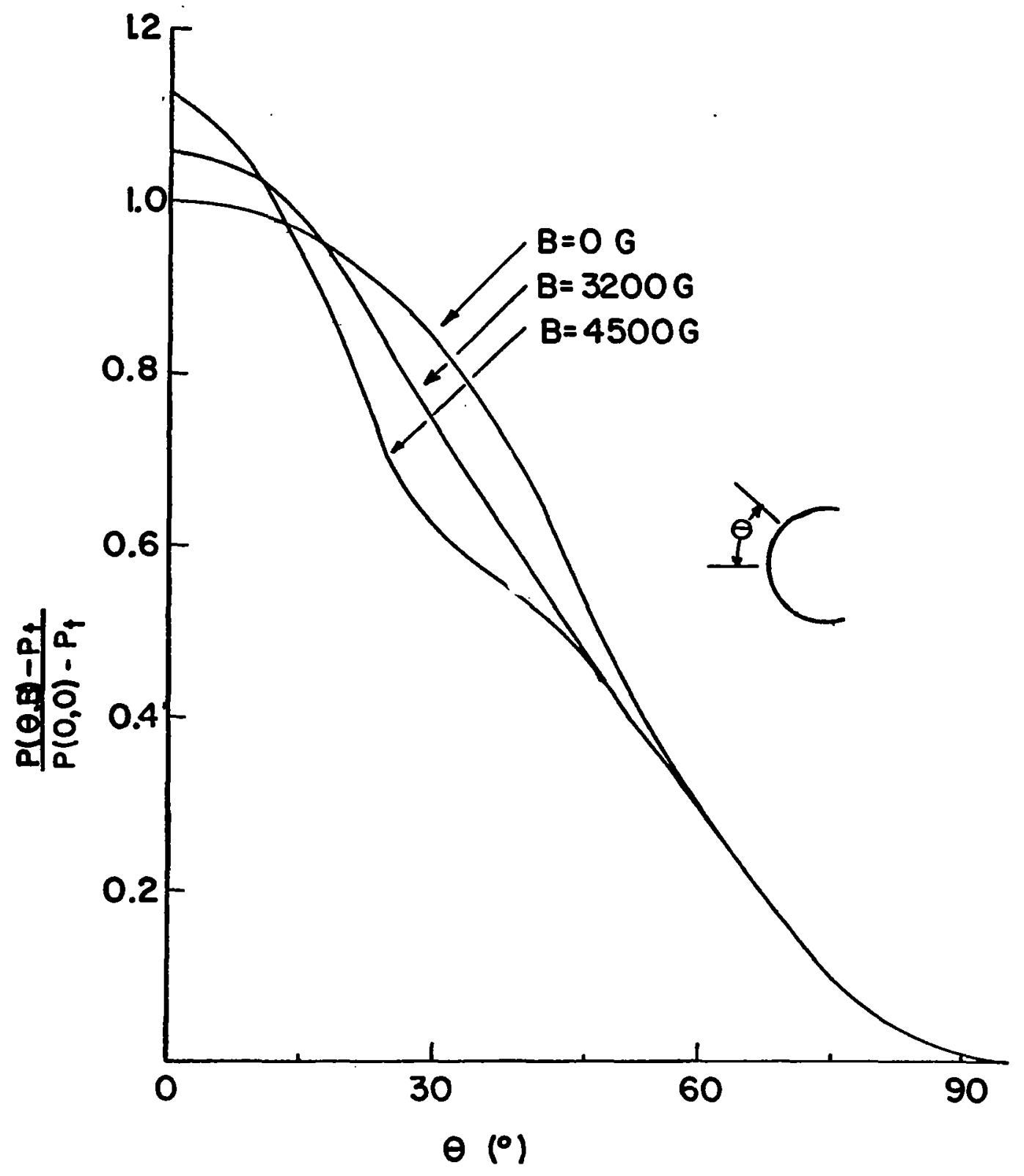

Fig. 17 Pressure distribution on body for several field strengths 


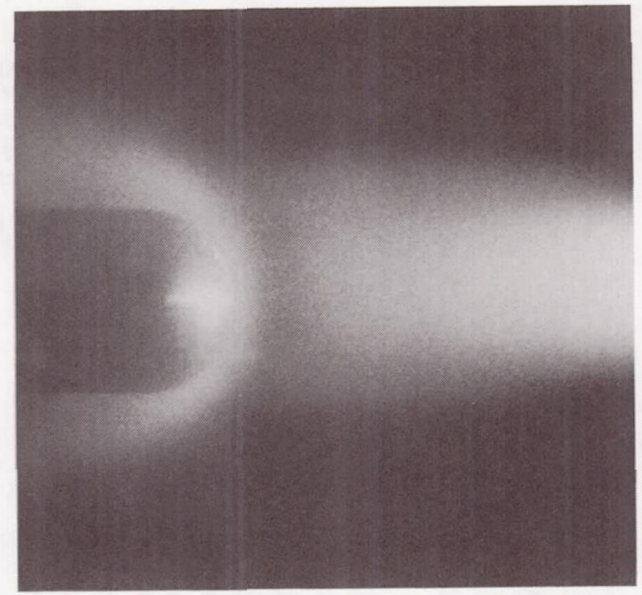

WITHOUT FIELD

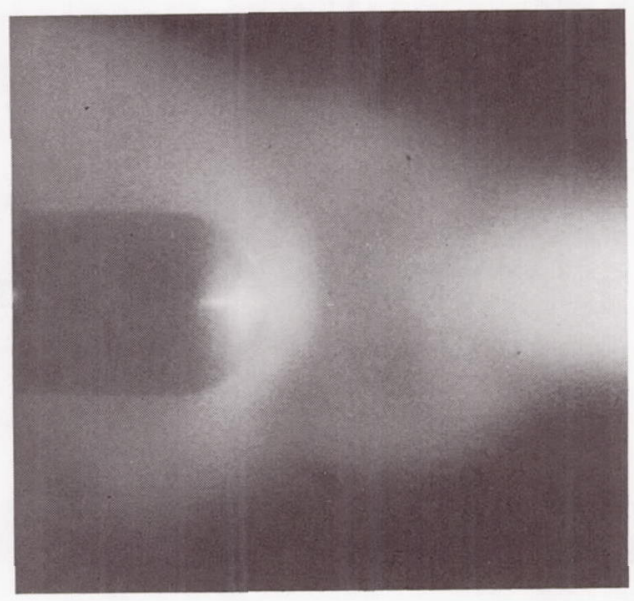

WITH FIELD $(B=4000 \mathrm{G})$

\section{HEMISPHERICAL BODY IN FLOW}

Fig. 18 Photograph of body in flow with and without magnetic field 


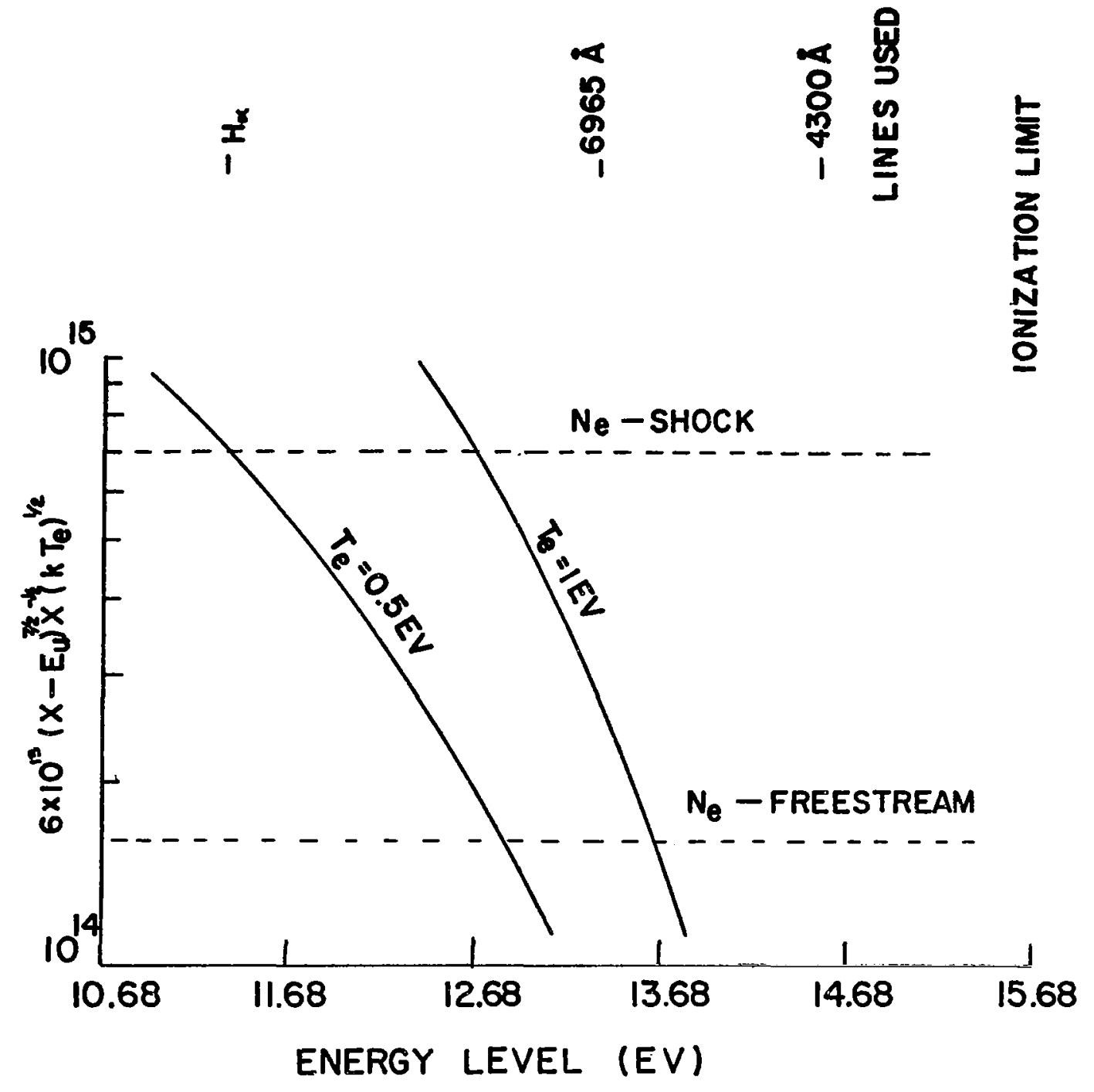

Fig. 19 Wilson's criterion for state equilibrium with free electrons 


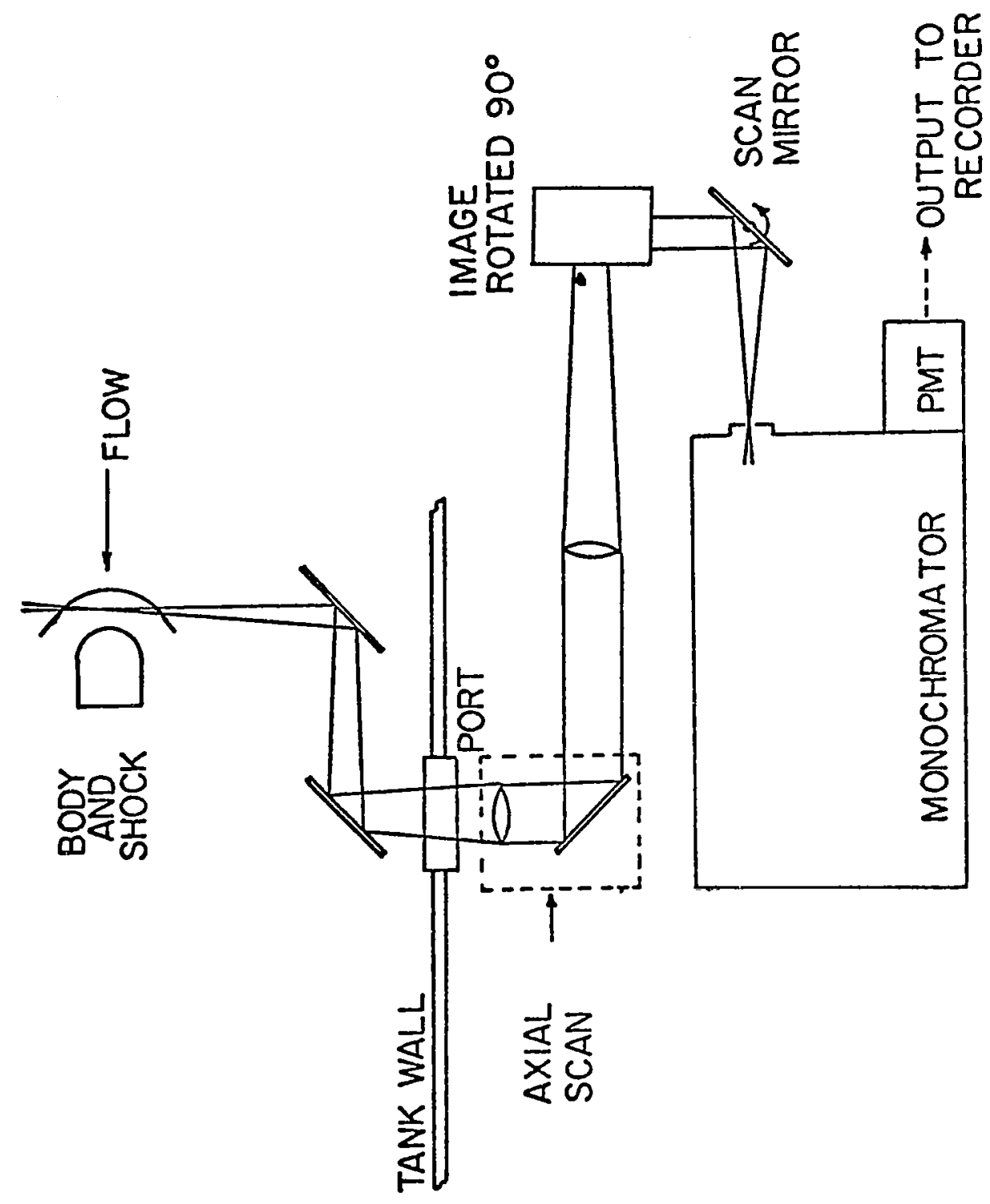

Fig. 20. Schematic of spectroscopic experiment. 


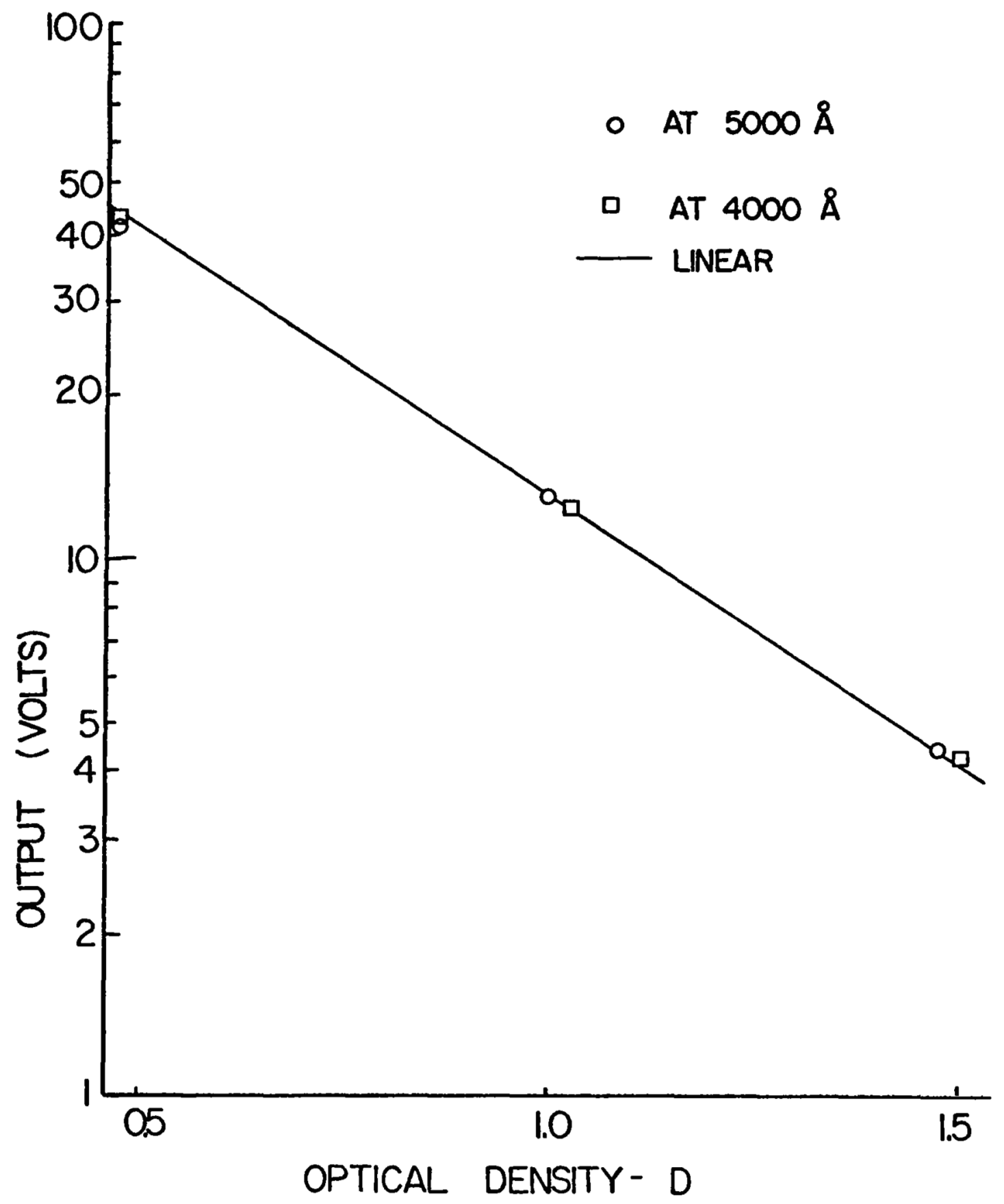

F1g. 21 Response of phototube 


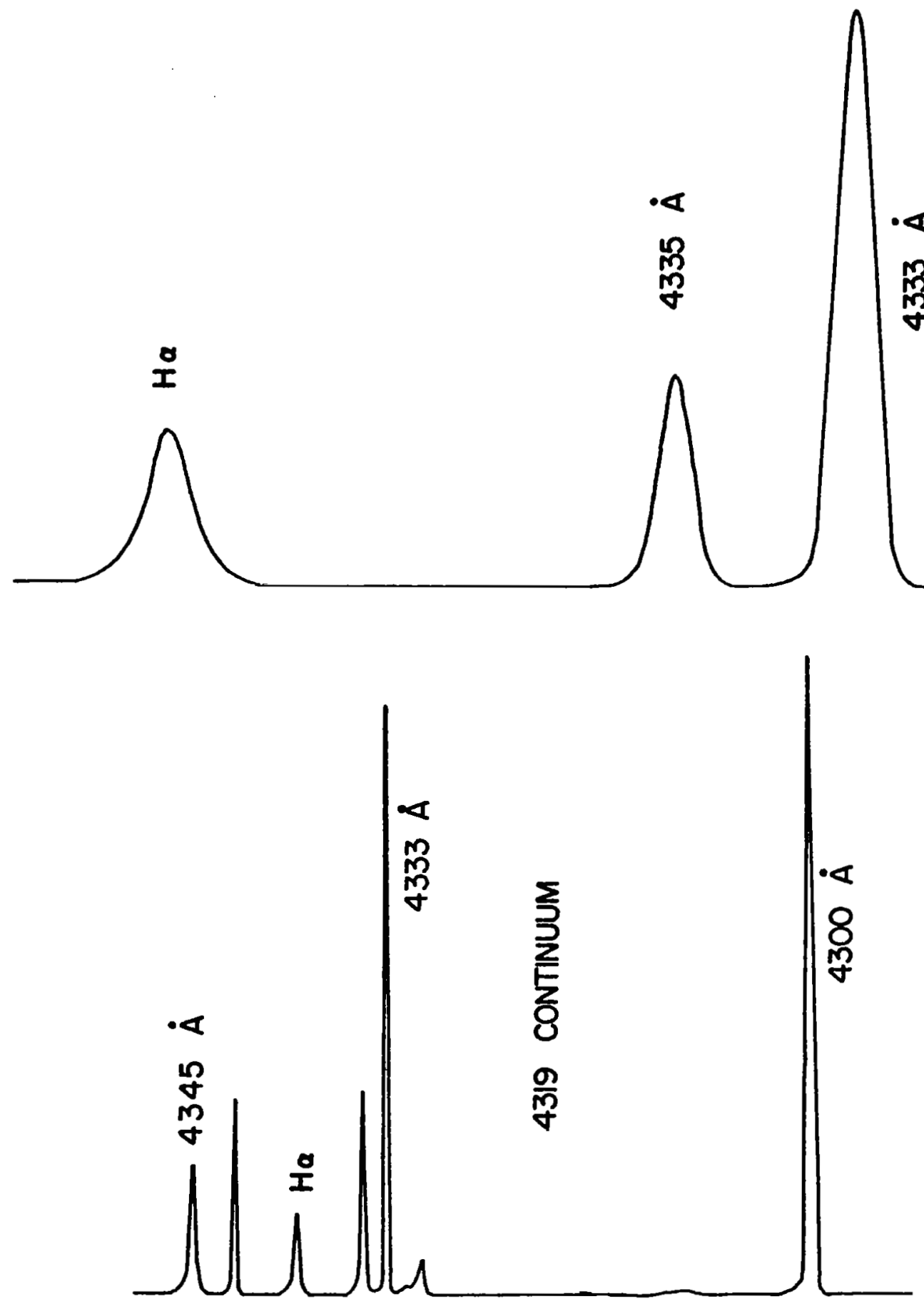

Fig. 22 Spectral scans showing continuum and some lines measured 


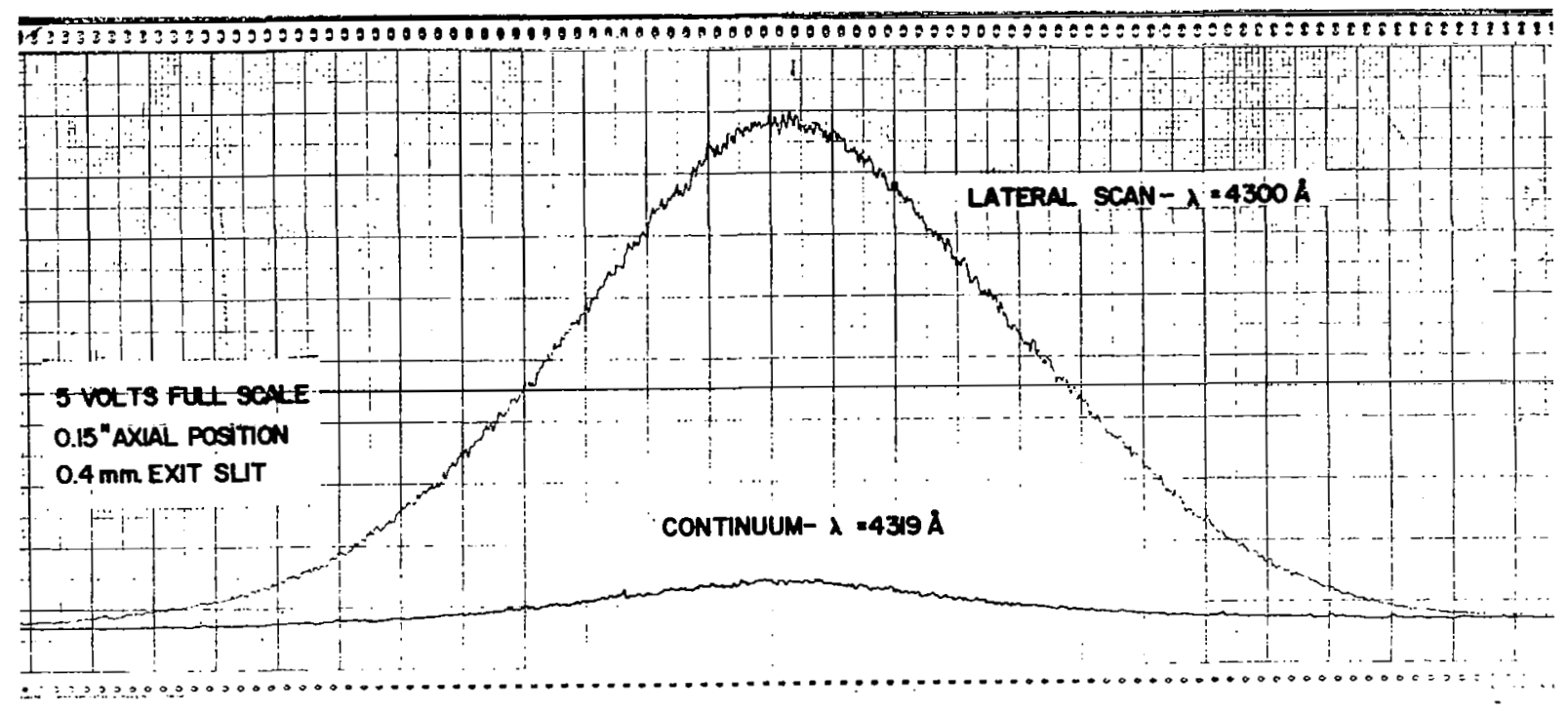

Fig. $234300 \AA$ line and underlying continuum 


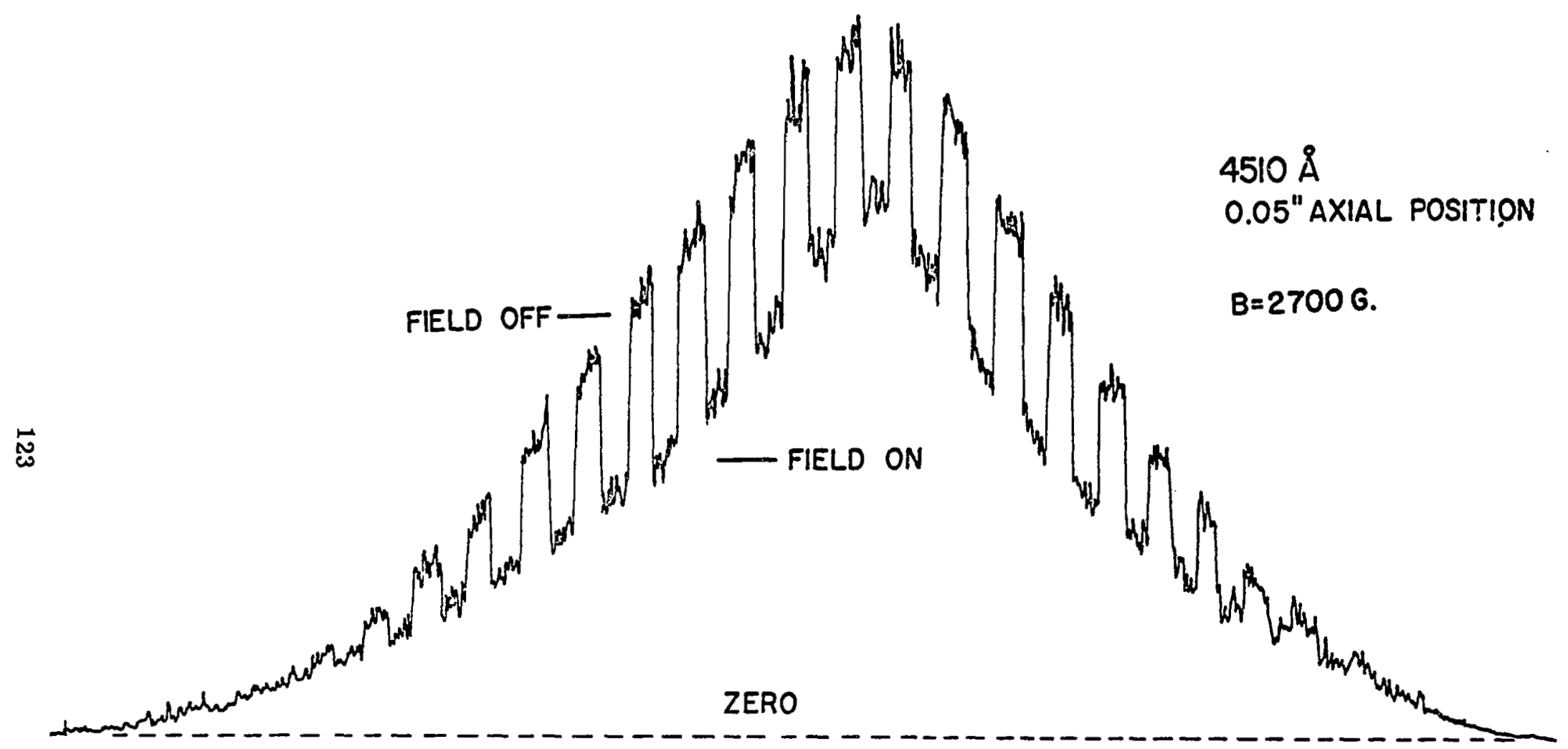

FIg. $244510 \AA$ Iine with and without magnetic field 


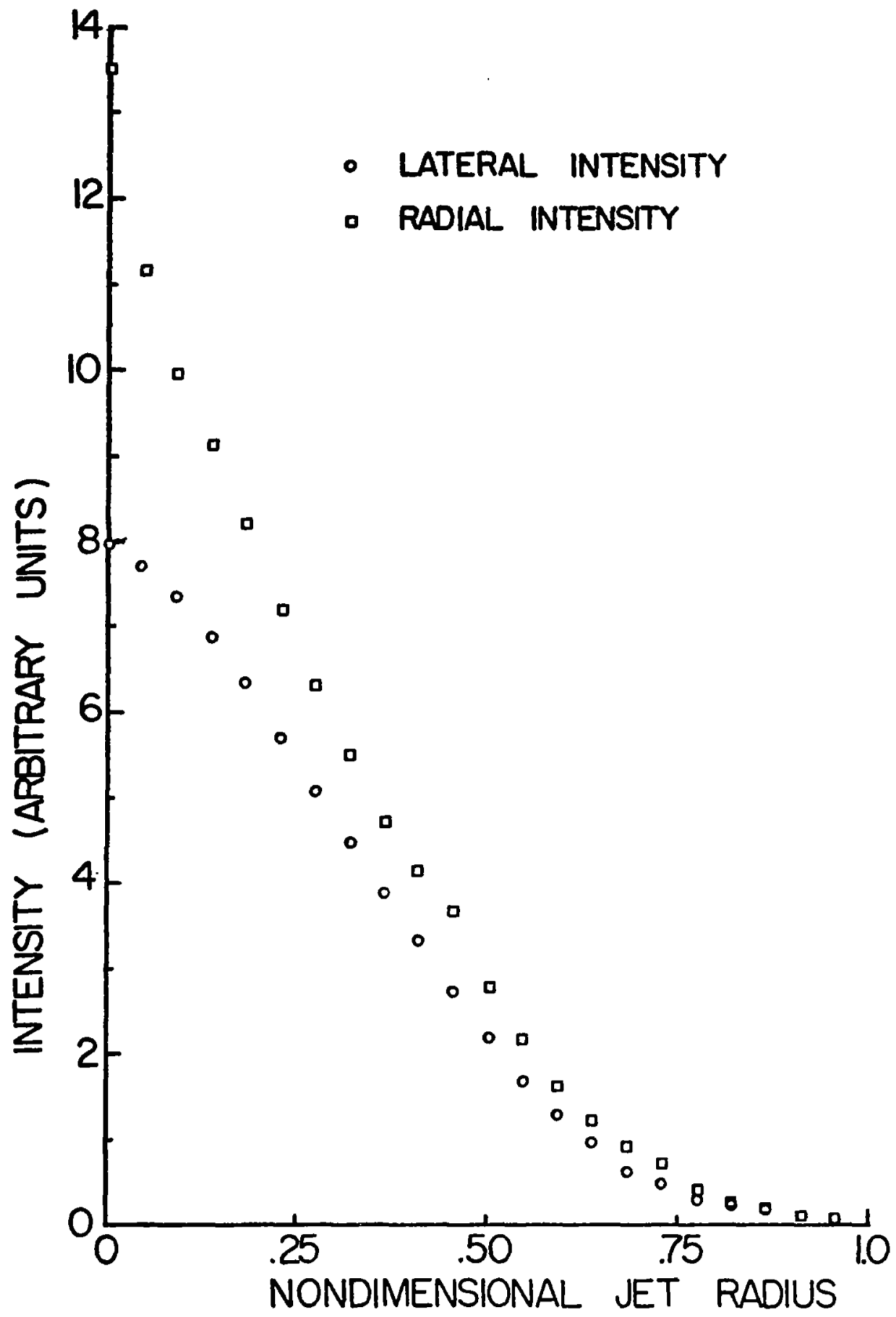

Fig. 25 Typical inversion of lateral scan 


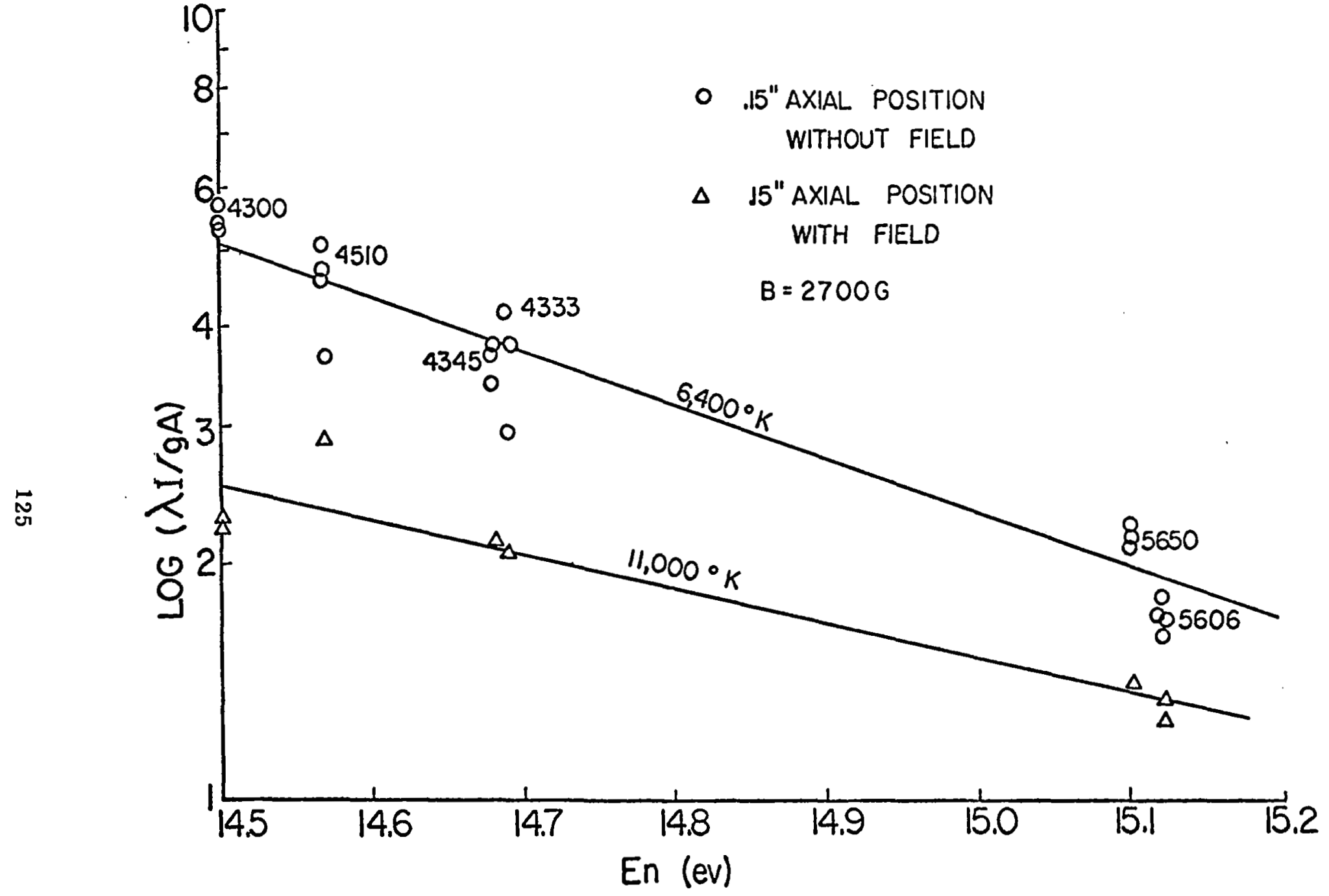

Fig. 26 Sample Boltzmann plot 


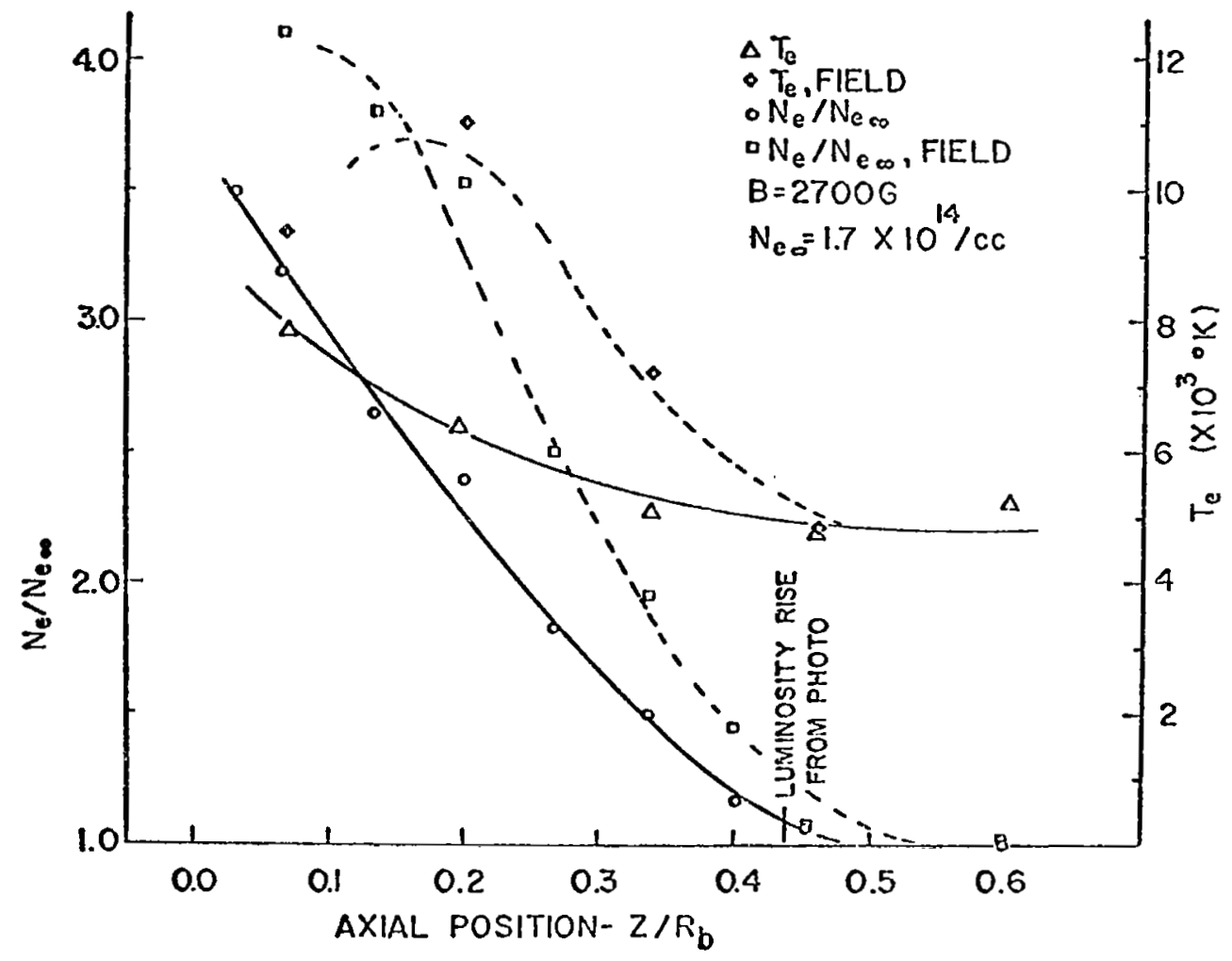

Fig. 27 Centerline electron temperature and body density with and without field 


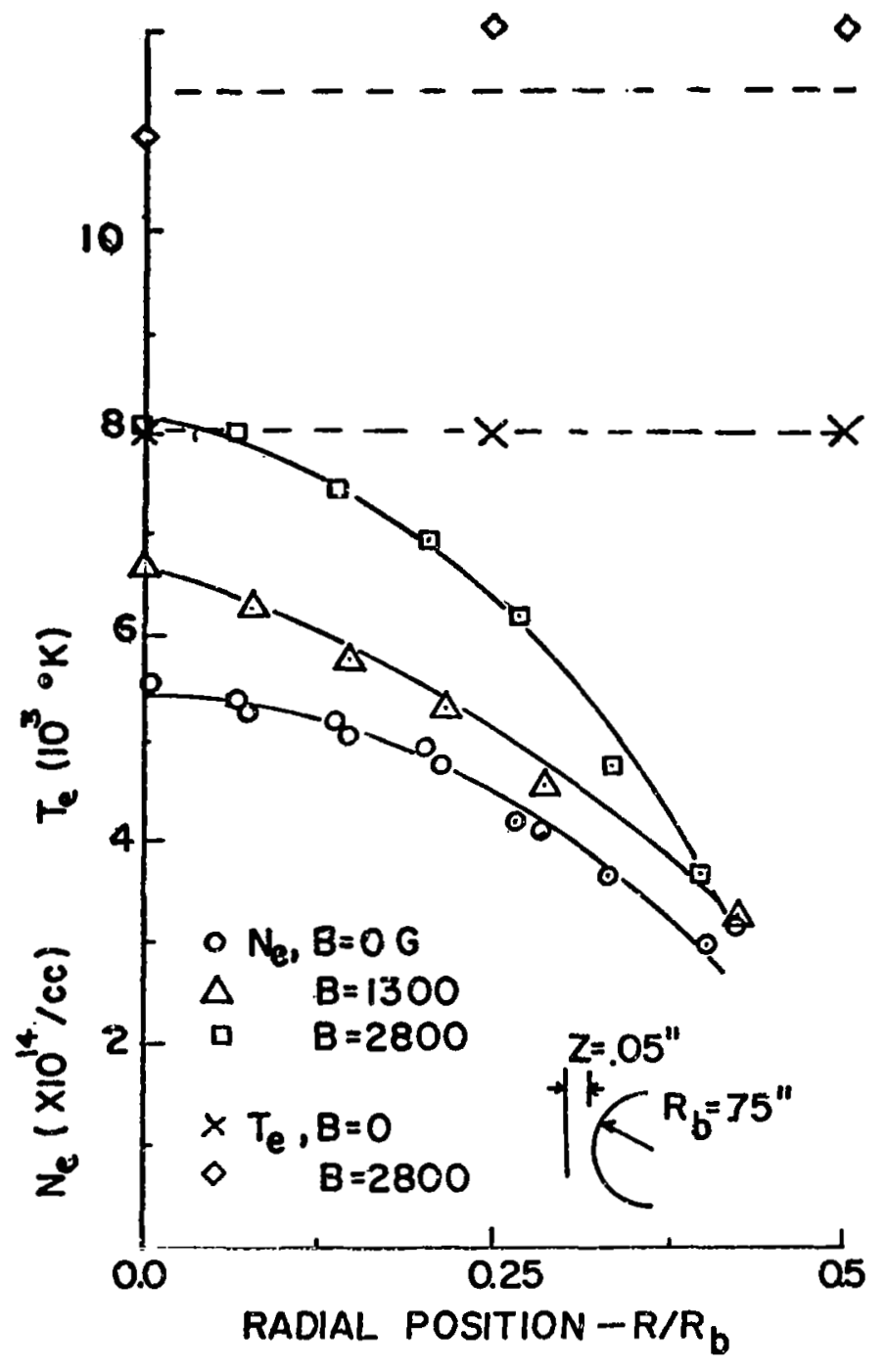

Fig. 28 Radial profiles of electron temperature and number density in the stagnation region 


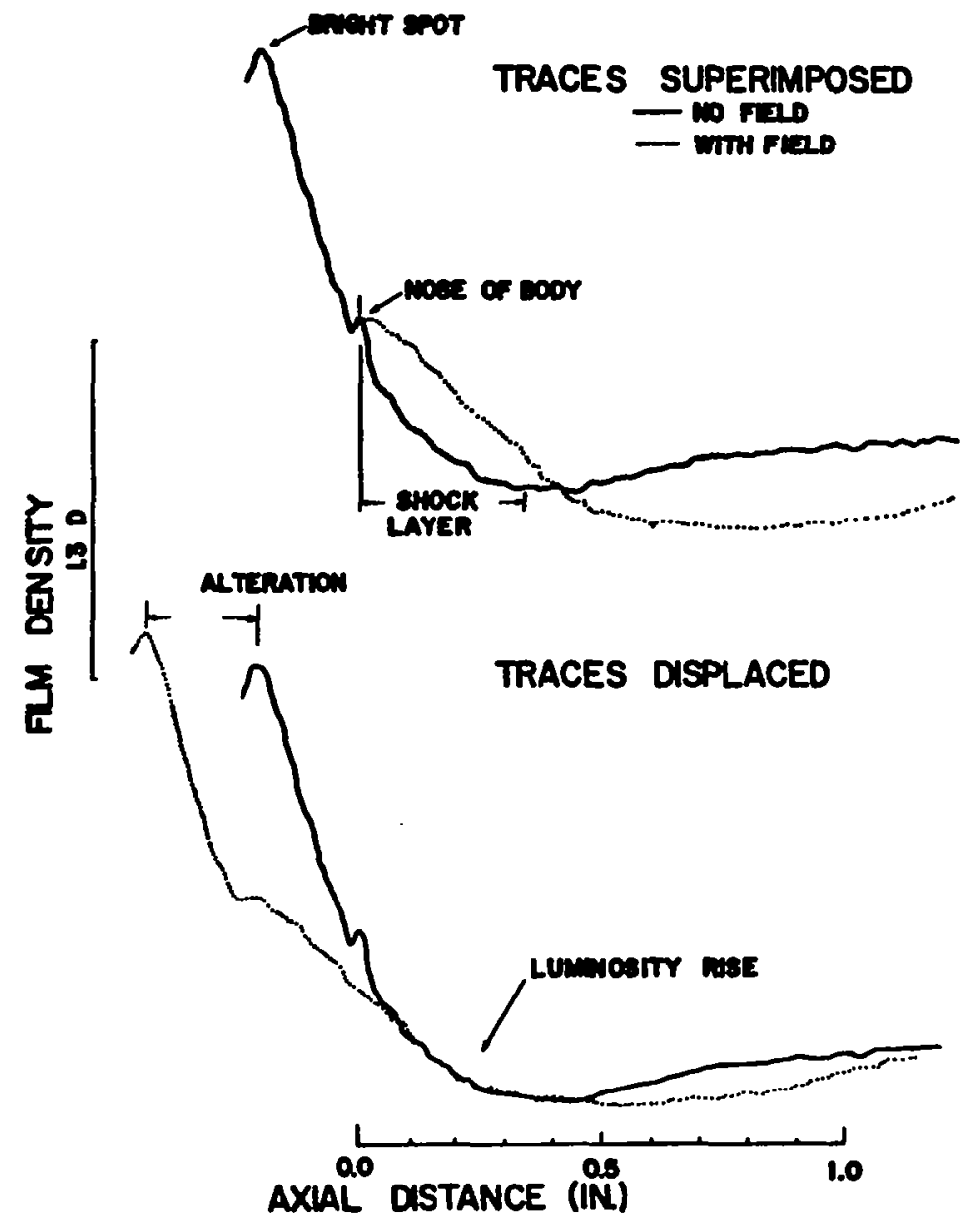

Fig. 29 Showing the photographic technique used to measure shock standoff alteration. 


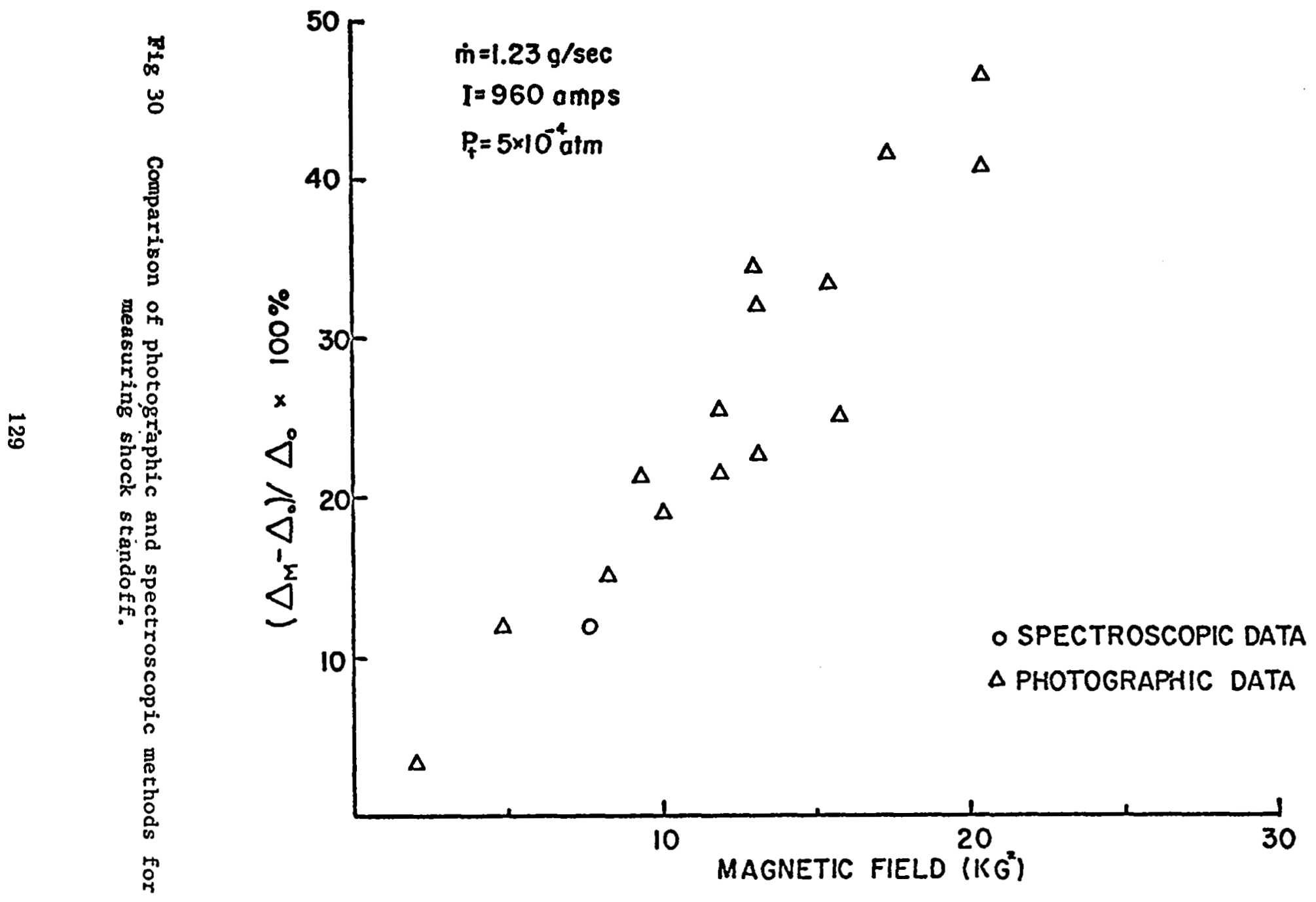




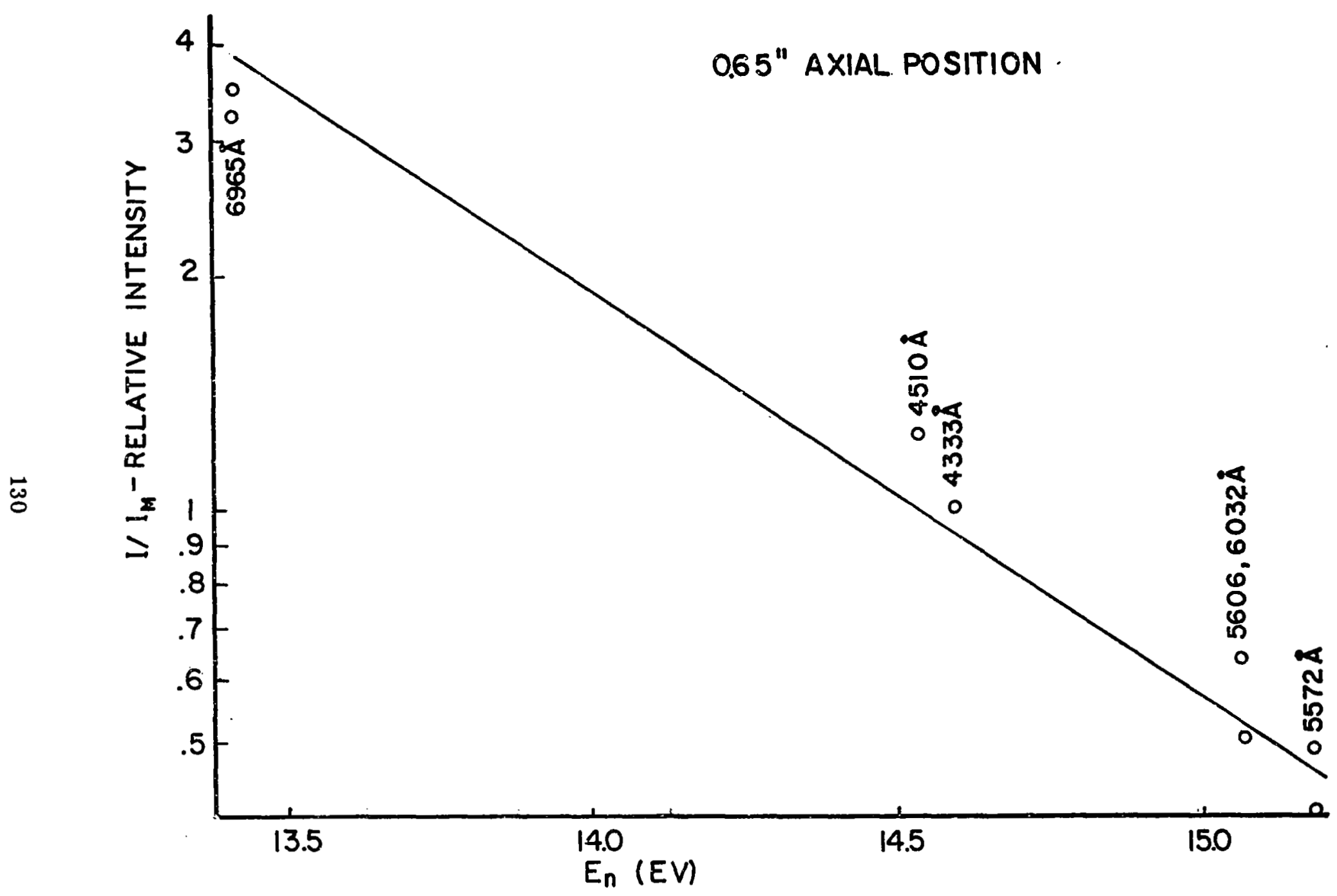

Fig. 31 Relative Boltzmann plot at $0.65^{\prime \prime}$ axial position 


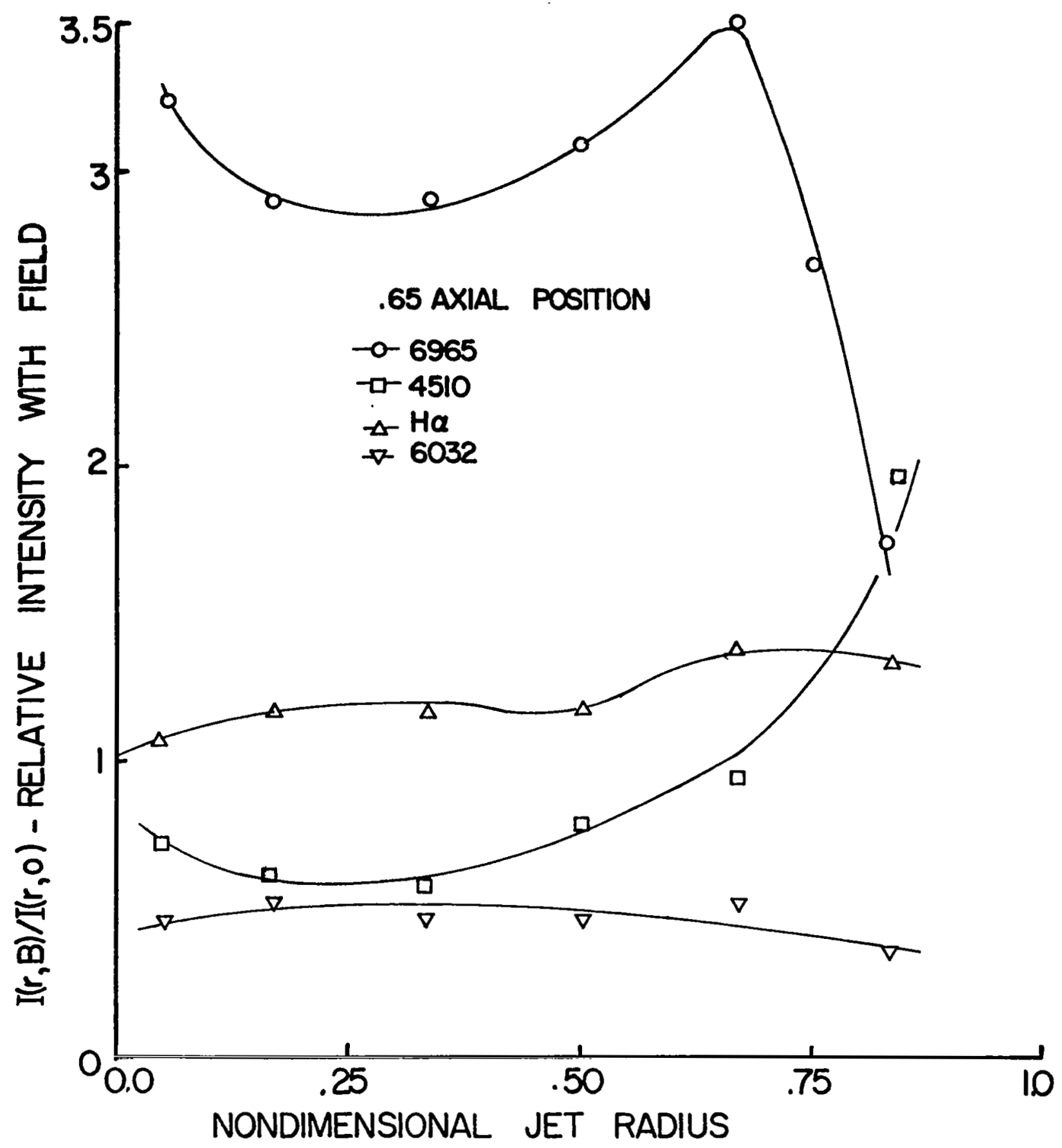

Fig. 32 Radial profiles of several spectral lines 


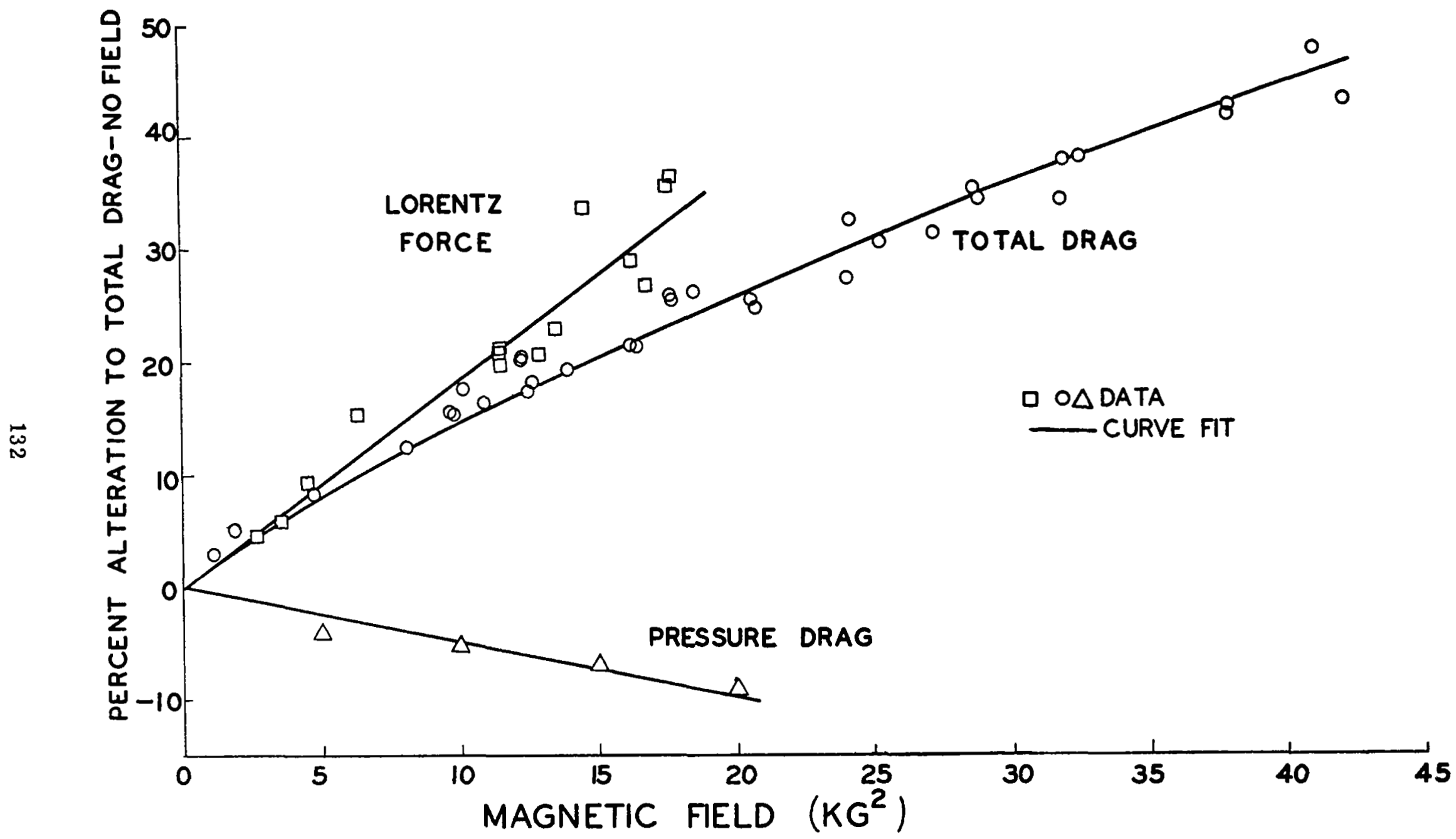

Fig. 33 Empirical relations for drag data 


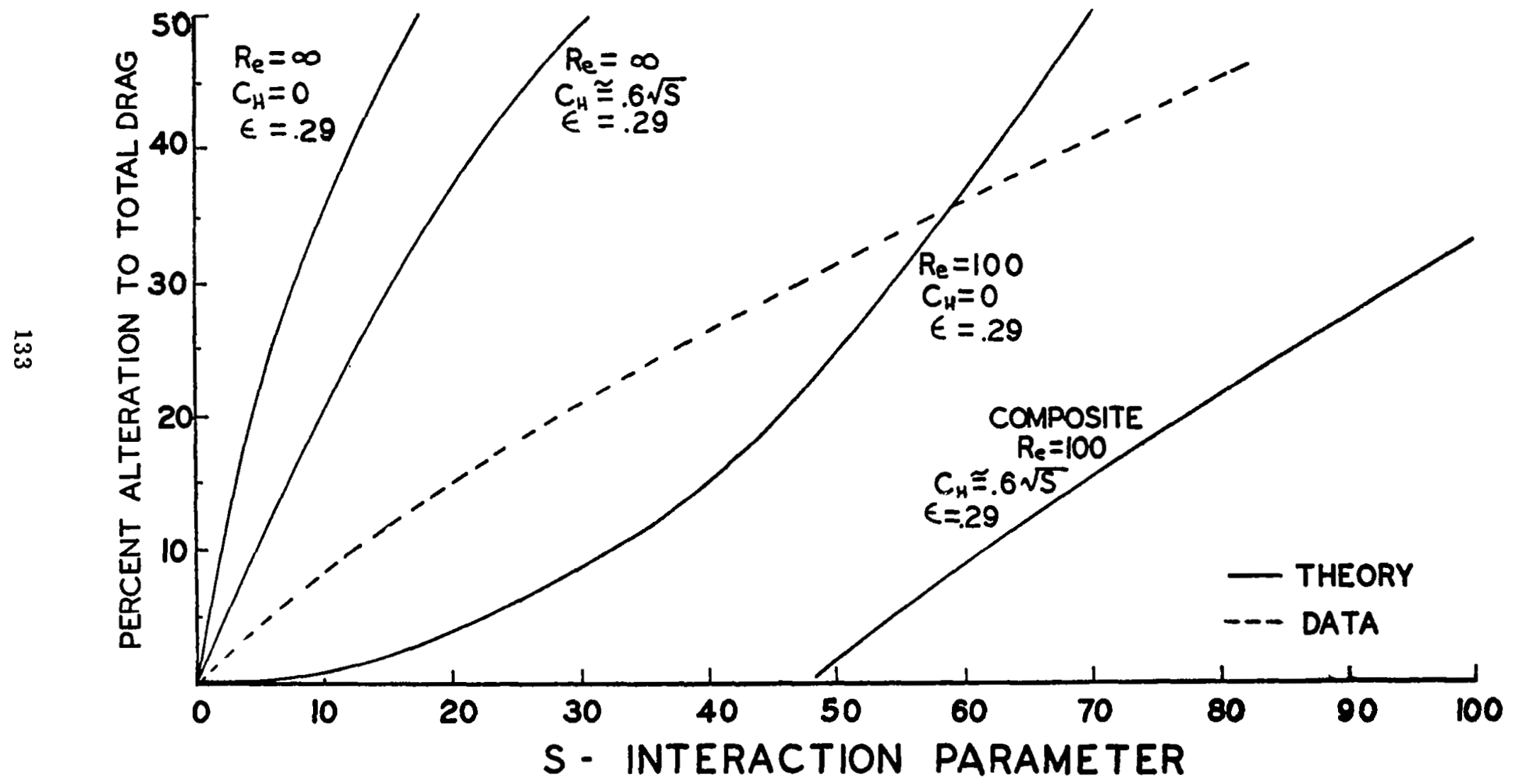

Fig. 34 Comparison of total drag data and theory 


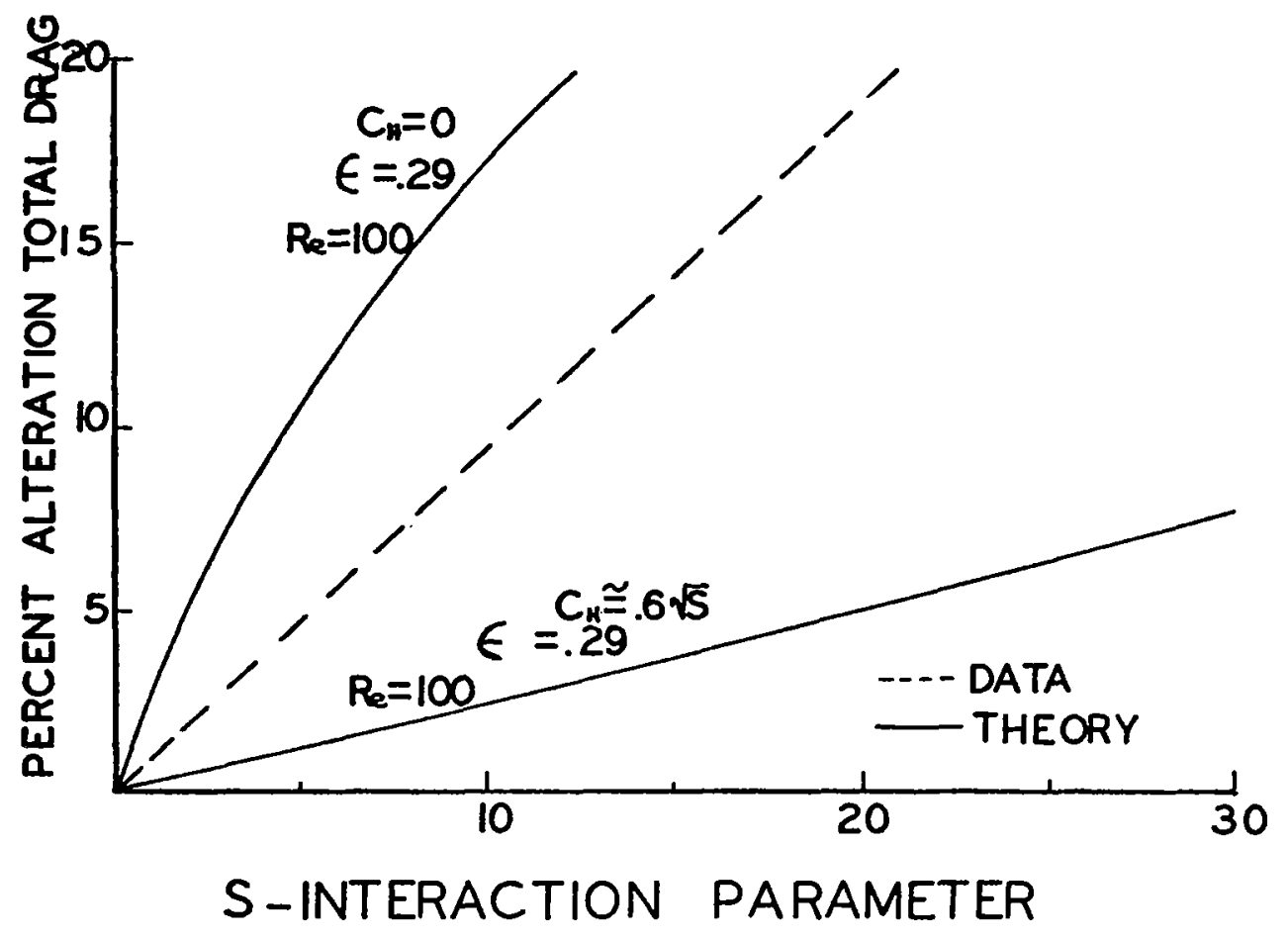

Fig. 35 Comparison of Lorentz drag data and theory 


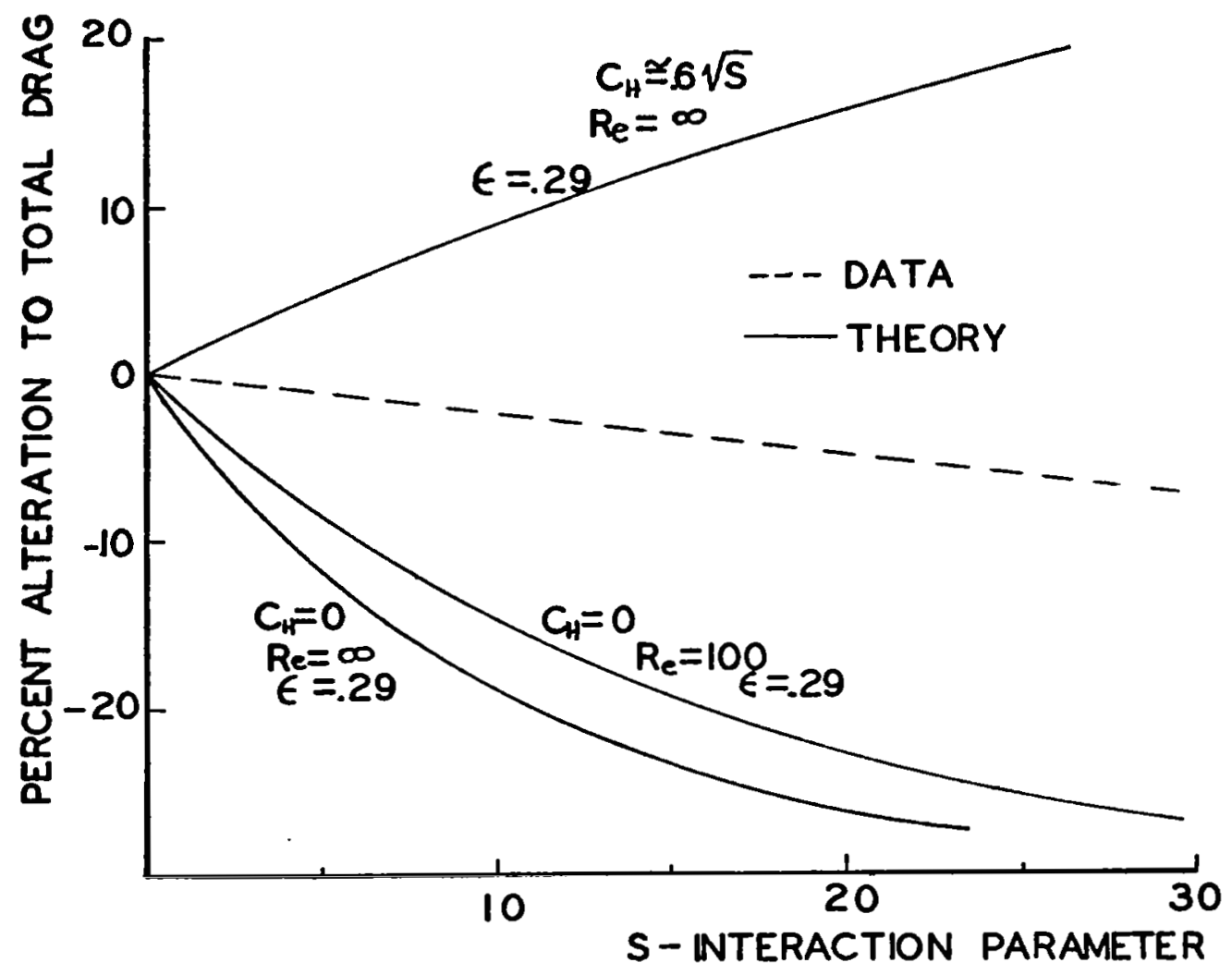

Fig. 36 Comparison of theoretical and experimental pressure force measurement 


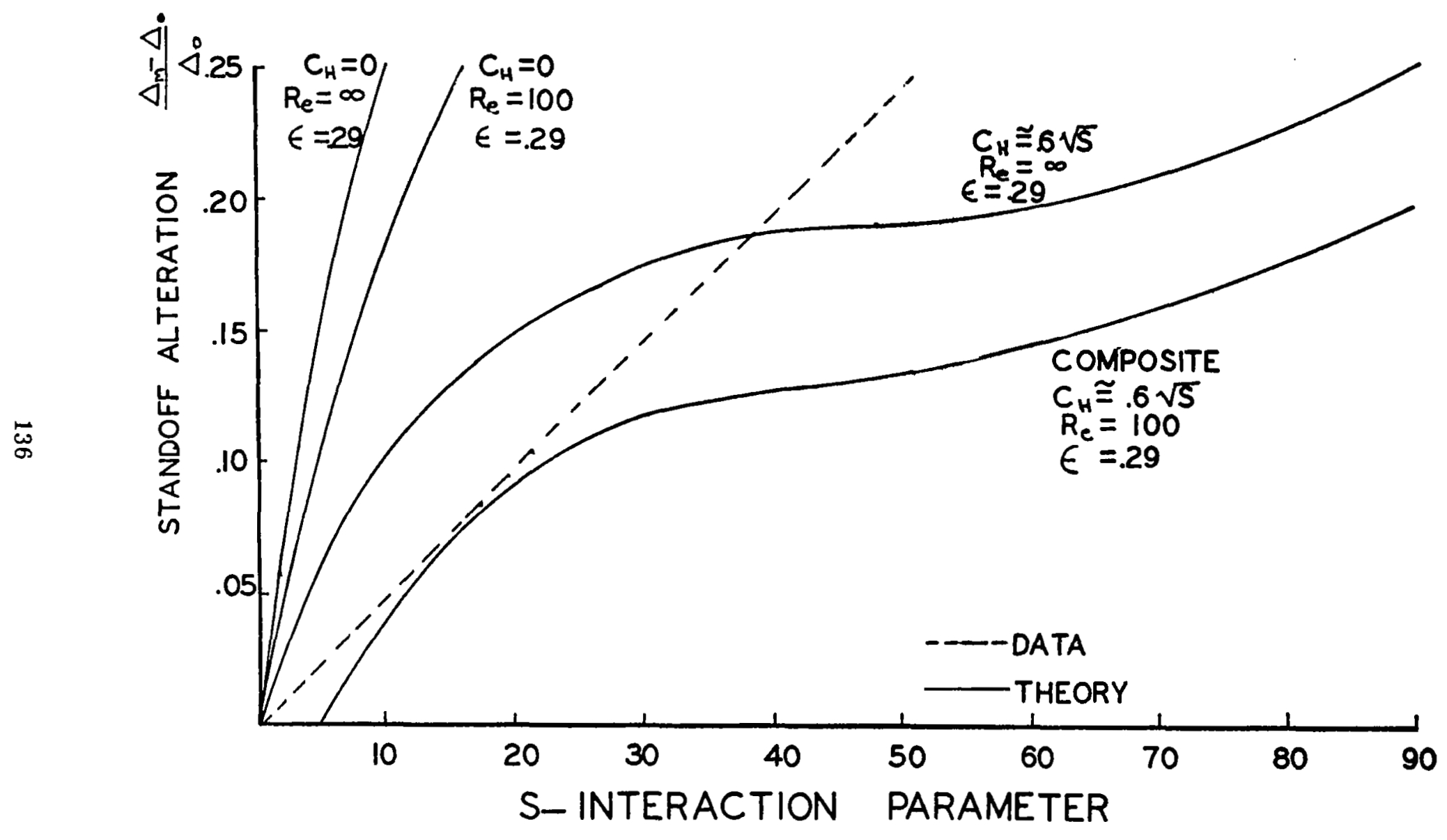

F1g. 37 Comparison of shock standoff data and theory 


\section{APPENDIX A}

\section{Facility}

A detailed schematic diagram of the facility is shown in Figure Al. The specifications for the equipment are as follows:

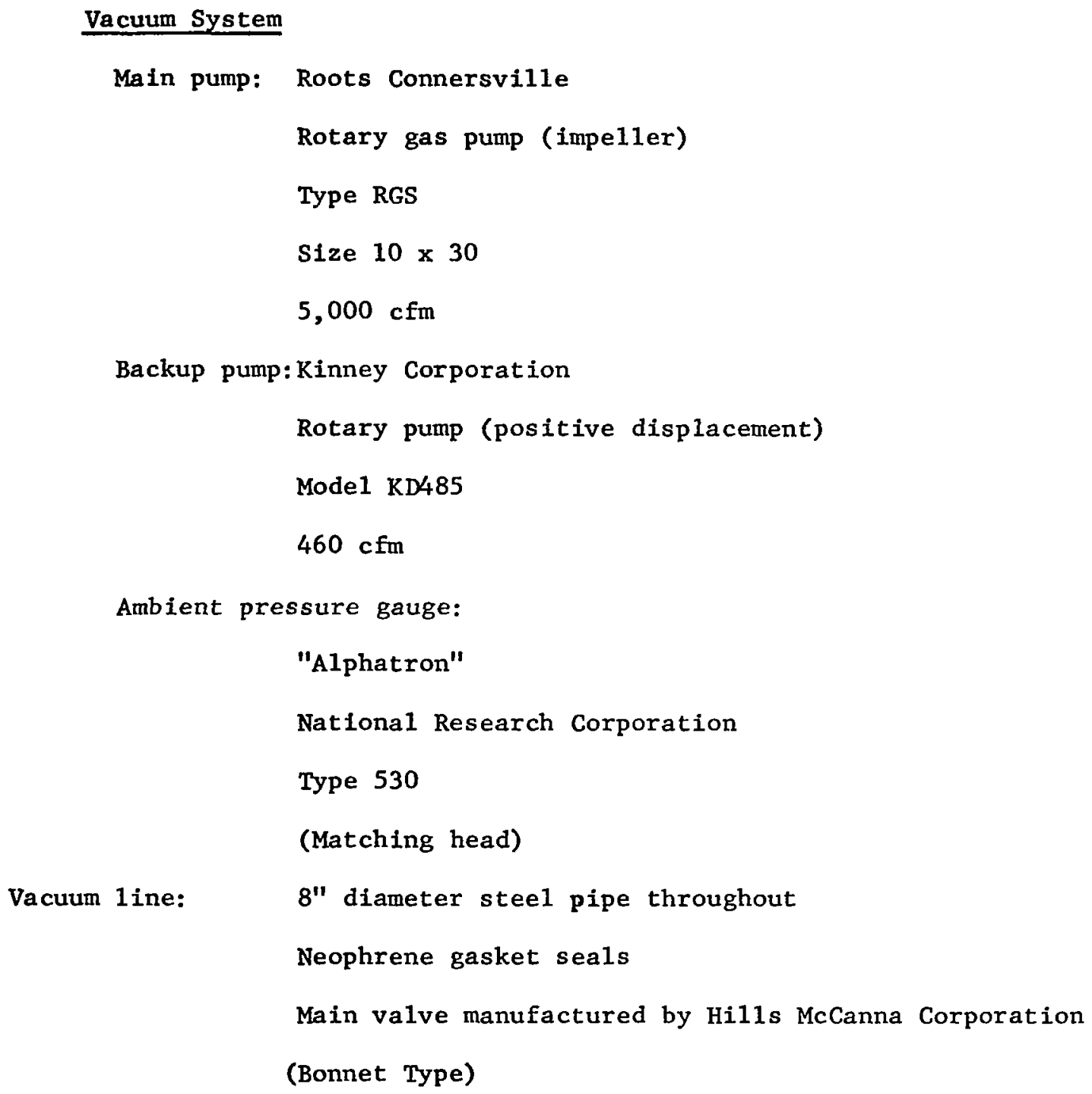




\section{Arc Heater and Power Supp1y}

Plasma Torch: Thermal Dynamics Corporation

Mode1 F80

(Maximum power input $80 \mathrm{KW}$ )

Standard electrode and anode $91 \mathrm{~B}, 1 / 2^{\prime \prime}$ diameter

Power Supply: Miller

(we1ders)

Mode1 S.R.H. .444

(4 units)

75 V.D.C., 400 amps, each

Gas Flowmeter: Fisher and Porter

\section{Flowrator}

Mode1 1 OA 3500

Some typical operation characteristics of the facility are shown in

Figure $A 2$ as a function of argon flow rate for a current setting of 960 amps. While a substantial change in ambient pressure could be caused by shutting off the blower pump the jet obtained was too small to be useful ( I" diameter).

Auxilliary equipment available for use with the facility included the following:

1. Davenport Power Supply, Mode1 VD2-30K-1E, $30 \mathrm{amp} / 200$ VDC (used for magnet power and battery charging).

2. Vo1ta Batteries - 60 amp hr. (storage type) -- 12v (32 used as main magnet power supply.)

3. Harrison Labs Mode1 6264A, 0-30 amp DC (constant current-constant voltage used for optical calibration). 
4. Fluke High Voltage Power Supply Mode1 405B, 0-2700 v/30 ma

(Photomultip1ier power supp1y).

5. Hewlett Packard/Dymec Scanning Digital Voltmeter:

Model 2401B Integrating Voltmeter

Model 2901A Input Scanner

Mode1 562A Digital Recorder

6. Moseley Two Channel Strip Chart Recorder, Type 7100A.

7. Bel1 Gaussmeter Mode1 120 (0-30 KG)

The drag measurement system described in Chapter IV is shown

schematically in Figure A3. The equipment specifications were as follows: ${ }^{1}$

1. Linear Differential Transformer:

Sanborn Mode1 FTA 100

Capacity 0-100 grams

Sensitivity $8 \mathrm{mv} / \mathrm{volt}$ applied

Linearity $0.2 \%$ fullscale

2. Power Supply and Demodulator:

Sanborn Transducer Converter Mode1 592

(operating at $2.4 \mathrm{KC} / 5$ volt $\mathrm{DC}$ input)

3. Recorder:

Moseley 7100A two channel strip chart recorder.

1. The optional filter circuit used to eliminate mechanical noise is described in "Andreyev's Circuit," Electronic Engineering, Vo1. 37, p. 444, February, 1965. 


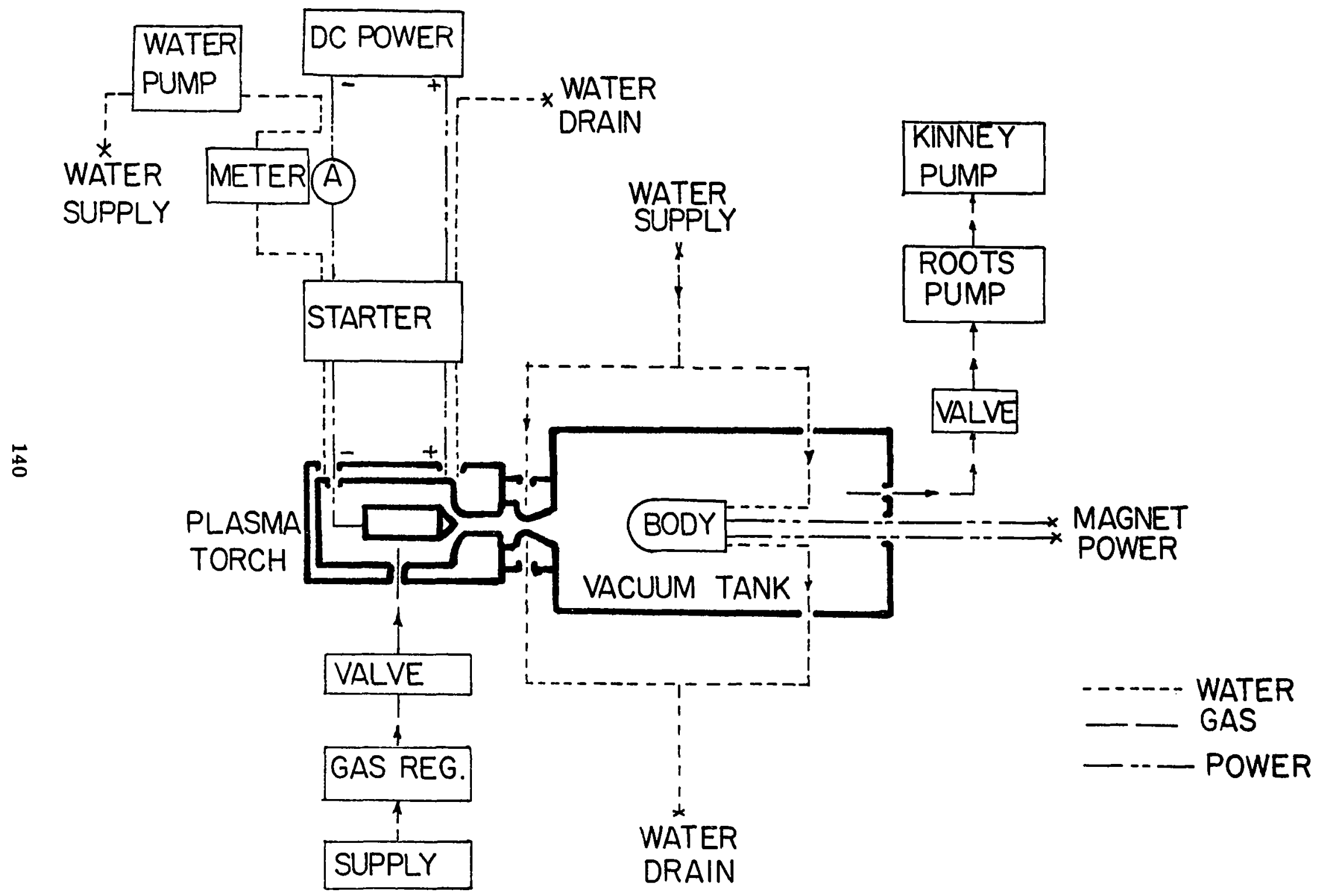

Fig. A1 Schematic of facility 


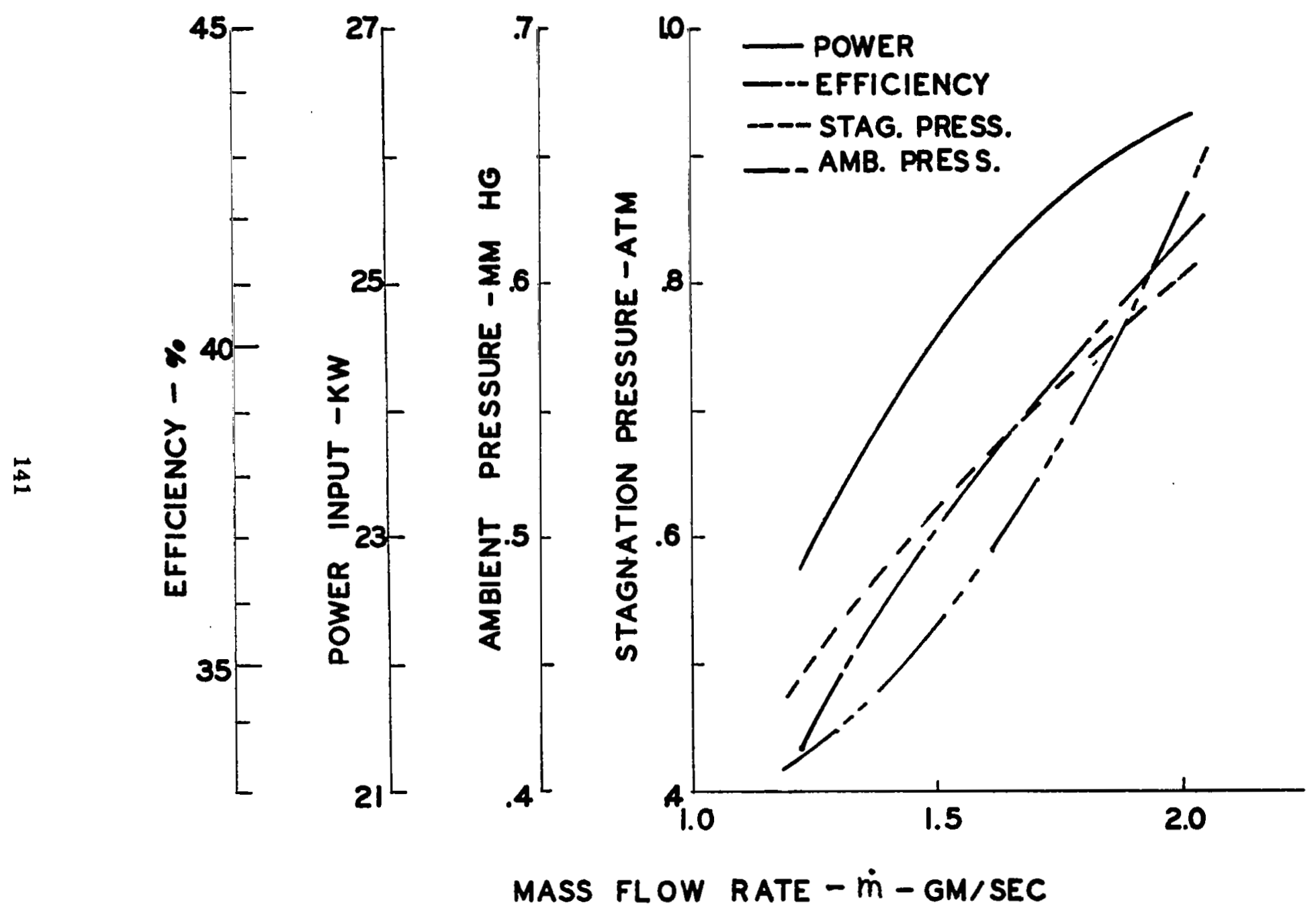

Fig. A2 Operating characteristics of faclity 


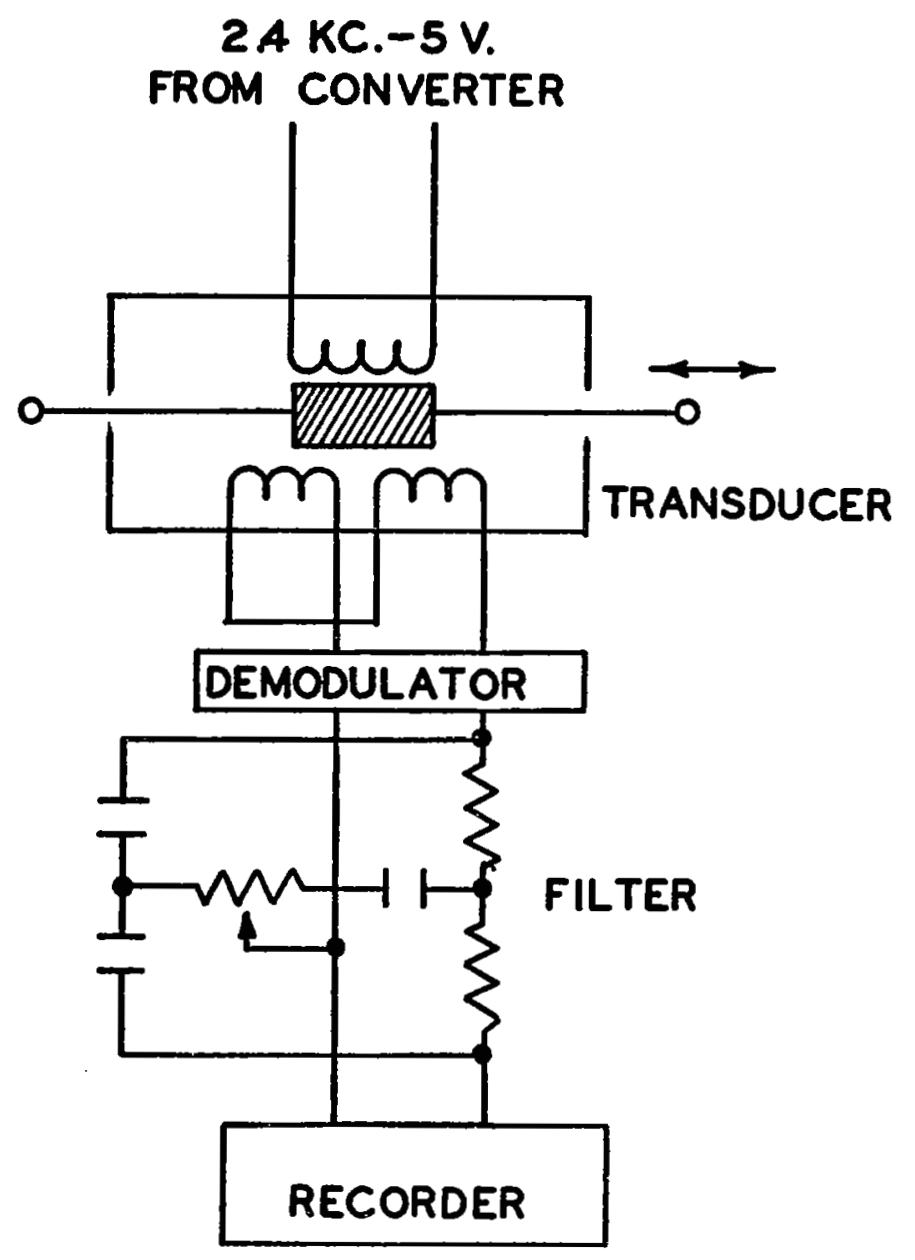

Fig. A3 Schematic of drag measuring device 


\section{APPENDIX B \\ Dlagnostic Measurement}

The method of determining impact and static pressure was discussed in Chapter III and the probes used for these measurements are shown in Figure 4. The interior detail of the sting support is shown in Figure B1.

The pressure sensor was a daiphram type, the displacement of the diaphram being read by a linear differential transformer mechantsm. The specifications are as follows:

Samborn Mode1 270 Differential Pressure Transducer:

Linearity $1 \%$ ful1 scale

Sensitivity $28 \mathrm{MV} / \mathrm{volt}$ applied

Minimum Differential Pressure $1.5 \mu$

Sanborn Transducer Converter Model 592:

(Operating at $2.4 \mathrm{KC} / 5$ volt $\mathrm{DC}$ input)

Some typical radial surveys made with the impact pressure probe under three different conditions are shown in Figure B2.

The mass flux probe tip is shown in Figure $B 3$ along with the schematic of the experimental arrangement. To measure the pressure in the exterior dump tank the following "Alphatron" gauge was used.

"Alphatron" vacuum gauge:

National Research Corporation

Mode1 520

(matching head) 
The spectroscope used was:

Hilger-Engis Mode1 1000

1 meter focal length

(Grating type monochrometer $5000 \AA$ blaze-1200 1ines/mm ( $8 \AA$ first order)) 


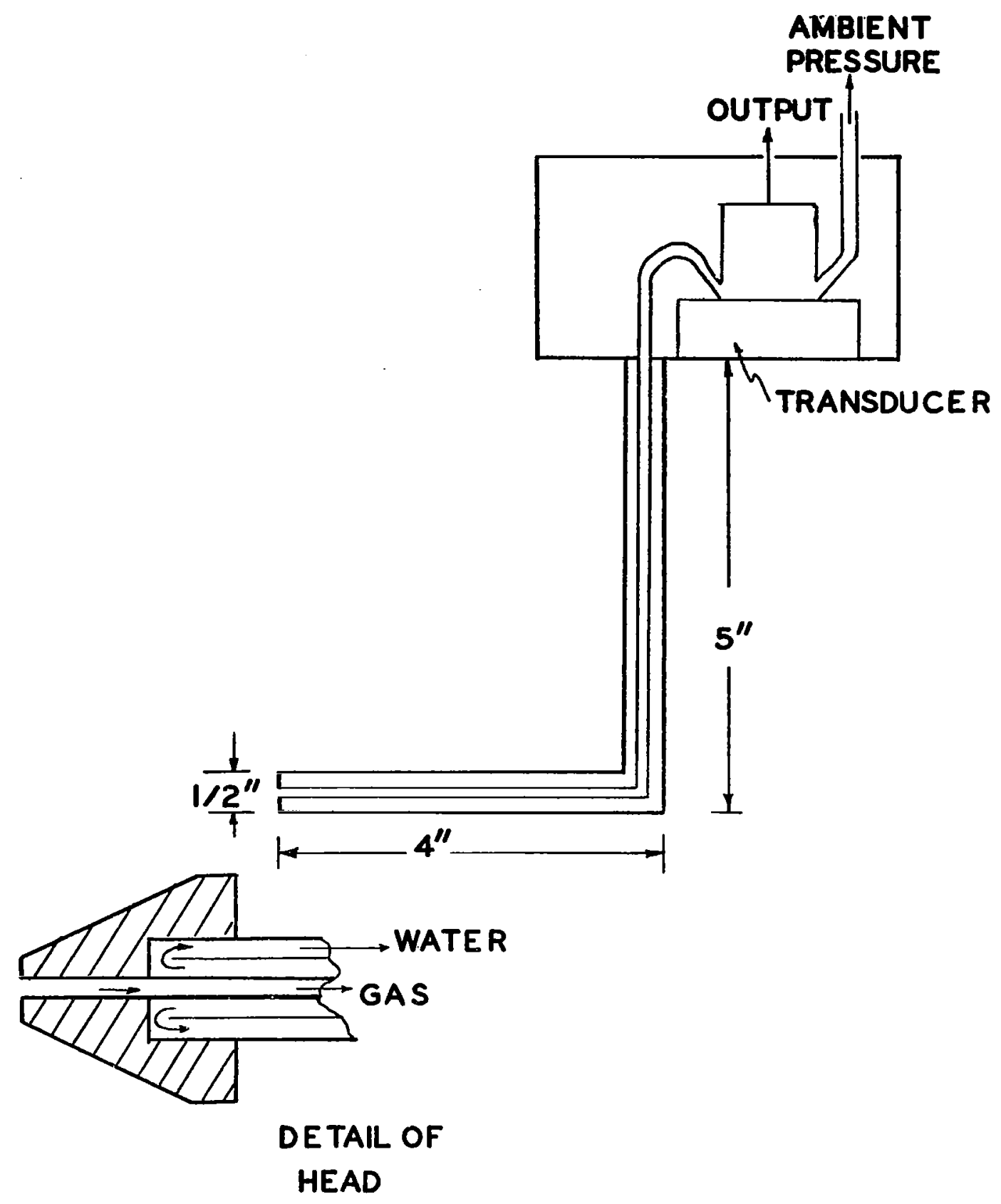

Fig. Bl Impact pressure probe -- interior detail 


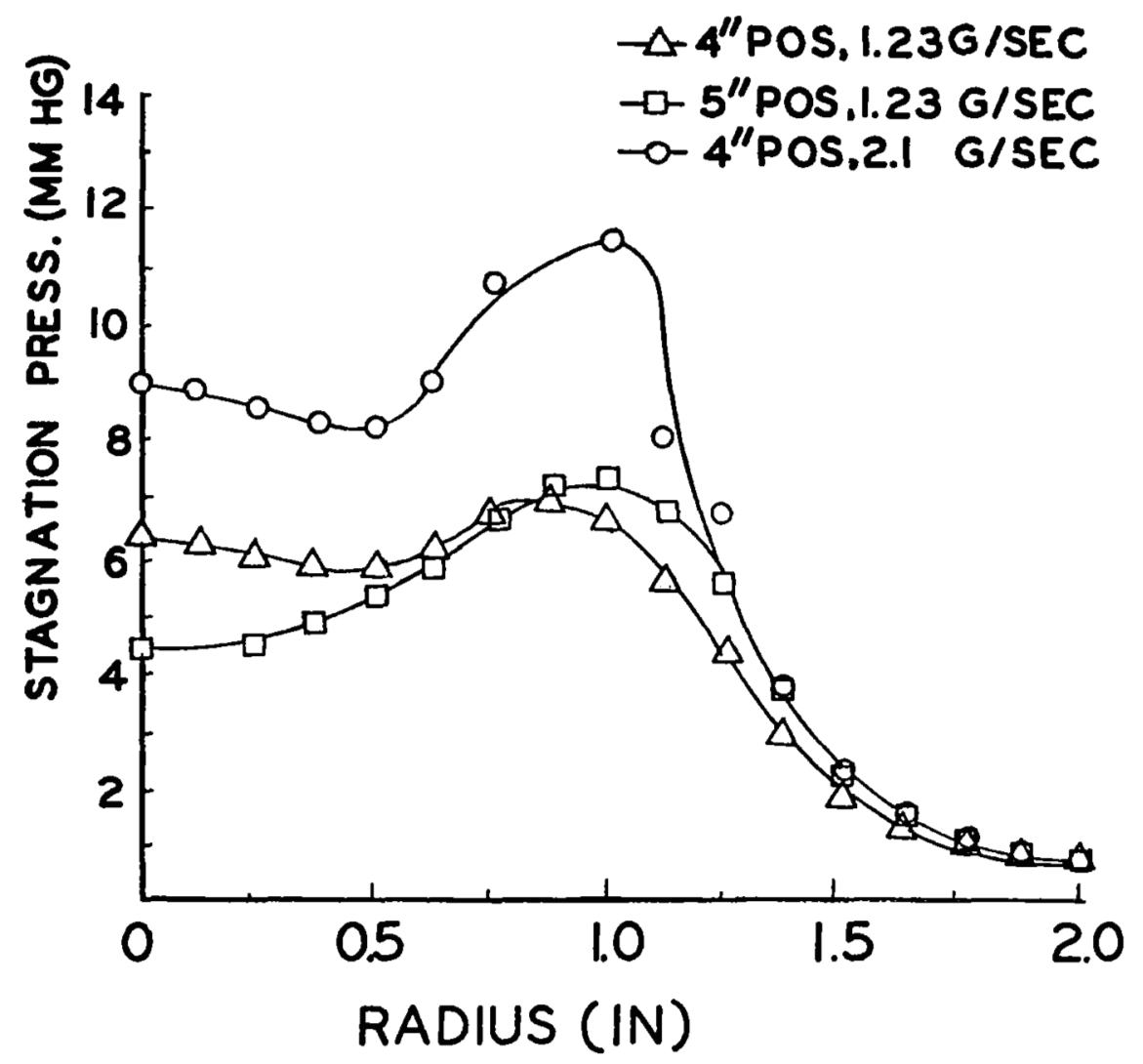

Fig. B2 Typical Impact pressure surveys 

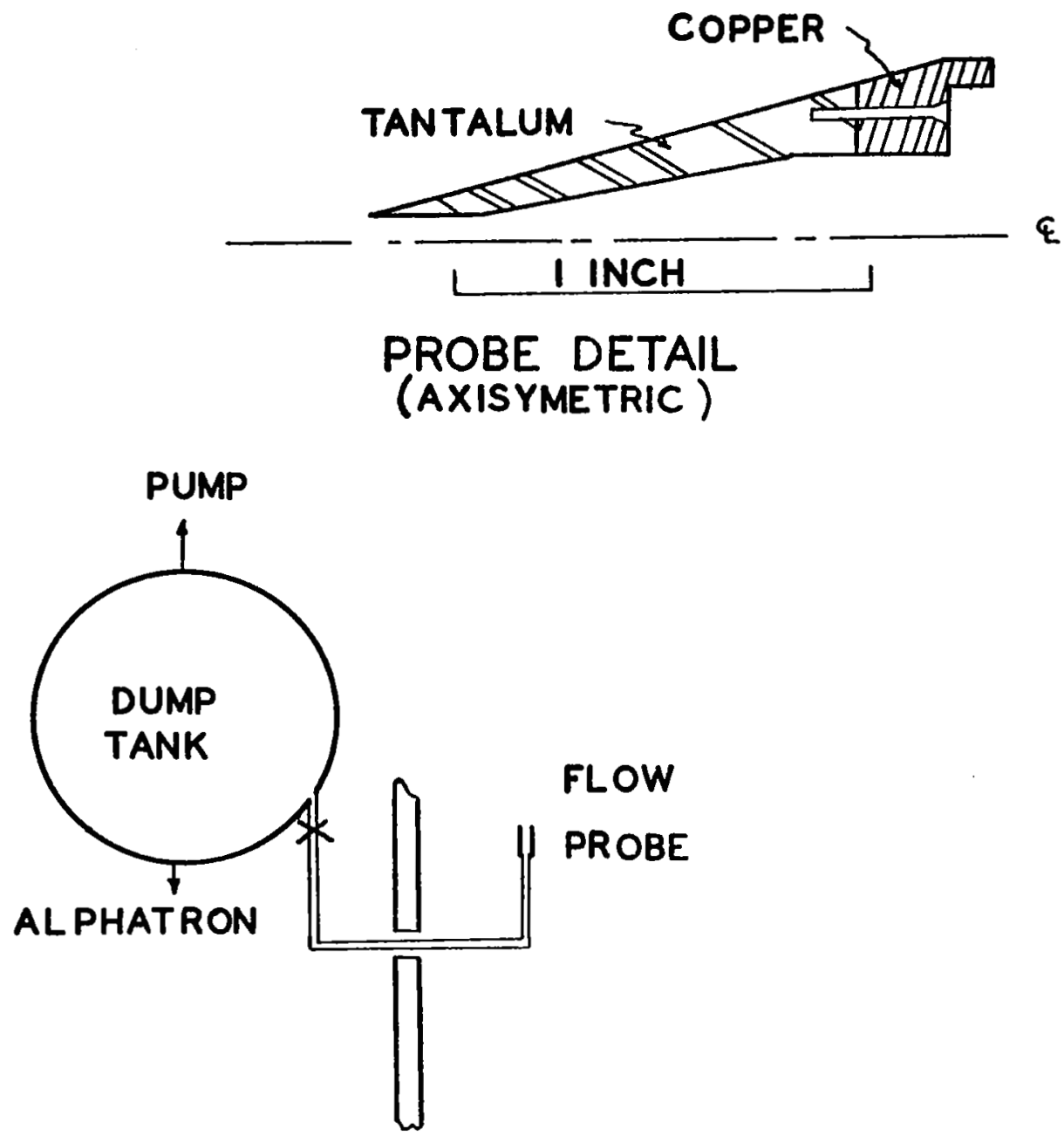

ARRANGEMENT

Fig. B3 Mass flux experiment -- detail. 